\title{
What is a cool-core cluster? a detailed analysis of the cores of the X-ray flux-limited HIFLUGCS cluster sample ${ }^{\star}$
}

\author{
D. S. Hudson ${ }^{1}$, R. Mittal ${ }^{1,2}$, T. H. Reiprich ${ }^{1}$, P. E. J. Nulsen ${ }^{3}$, H. Andernach ${ }^{1,4}$, and C. L. Sarazin ${ }^{5}$ \\ ${ }^{1}$ Argelander-Institut für Astronomie der Universität Bonn, Auf dem Hügel 71, 53121 Bonn, Germany \\ e-mail: rmittal@astro.rit.edu \\ 2 Rochester Institute of Technology, 84 Lamb Memorial Drive, Rochester, NY 14623, USA \\ 3 Harvard-Smithsonian Center for Astrophysics, 60 Garden Street, Cambridge, MA 02138, USA \\ 4 on leave of absence from Departamento de Astronomía, Universidad de Guanajuato, AP 144, Guanajuato CP 36000, Mexico \\ 5 Department of Astronomy, University of Virginia, PO Box 400325, Charlottesville, VA 22904-4325, USA
}

Received 23 April 2009 / Accepted 20 October 2009

\section{ABSTRACT}

\begin{abstract}
We use the largest complete sample of 64 galaxy clusters (HIghest X-ray FLUx Galaxy Cluster Sample) with available high-quality $\mathrm{X}$-ray data from Chandra, and apply 16 cool-core diagnostics to them, some of them new. In order to identify the best parameter for characterizing cool-core clusters and quantify its relation to other parameters, we mainly use very high spatial resolution profiles of central gas density and temperature, and quantities derived from them. We also correlate optical properties of brightest cluster galaxies (BCGs) with X-ray properties.

To segregate cool core and non-cool-core clusters, we find that central cooling time, $t_{\text {cool }}$, is the best parameter for low redshift clusters with high quality data, and that cuspiness is the best parameter for high redshift clusters. $72 \%$ of clusters in our sample have a cool core $\left(t_{\text {cool }}<7.7 h_{71}^{-1 / 2} \mathrm{Gyr}\right)$ and $44 \%$ have strong cool cores $\left(t_{\text {cool }}<1.0 h_{71}^{-1 / 2} \mathrm{Gyr}\right)$. We find strong cool-core clusters are characterized as having low central entropy and a systematic central temperature drop. Weak cool-core clusters have enhanced central entropies and temperature profiles that are flat or decrease slightly towards the center. Non-cool-core clusters have high central entropies.

For the first time we show quantitatively that the discrepancy in classical and spectroscopic mass deposition rates can not be explained with a recent formation of the cool cores, demonstrating the need for a heating mechanism to explain the cooling flow problem.

We find that strong cool-core clusters have a distribution of central temperature drops, centered on $0.4 T_{\text {vir }}$. However, the radius at which the temperature begins to drop varies. This lack of a universal inner temperature profile probably reflects the complex physics in cluster cores not directly related to the cluster as a whole. Our results suggest that the central temperature does not correlate with the mass of the BCGs and weakly correlates with the expected radiative cooling only for strong cool-core clusters. Since $88 \%$ of the clusters in our sample have a BCG within a projected distance of $50 h_{71}^{-1} \mathrm{kpc}$ from the X-ray peak, we argue that it is easier to heat the gas (e.g. with mergers or non-gravitational processes) than to separate the dense core from the brightest cluster galaxy.

Diffuse, Mpc-scale radio emission, believed to be associated with major mergers, has not been unambiguously detected in any of the strong cool-core clusters in our sample. Of the weak cool-core clusters and non-cool-core clusters, most of the clusters (seven out of eight) that have diffuse, Mpc-scale radio emission have a large ( $>50 h_{71}^{-1} \mathrm{kpc}$ ) projected separation between their BCG and X-ray peak. In contrast, only two of the 56 clusters with a small separation between the BCG and X-ray peak $\left(<50 h_{71}^{-1} \mathrm{kpc}\right)$ show large-scale radio emission. Based on this result, we argue that a large projected separation between the BCG and the X-ray peak is a good indicator of a major merger. The properties of weak cool-core clusters as an intermediate class of objects are discussed. Finally we describe individual properties of all 64 clusters in the sample.
\end{abstract}

Key words. intergalactic medium - galaxies: clusters: general

\section{Introduction}

Early X-ray observations of galaxy clusters revealed that the intracluster medium (ICM) in the centers of many clusters was so dense that the cooling time of the gas was much shorter than the Hubble time (e.g. Lea et al. 1973; Cowie \& Binney 1977; Fabian \& Nulsen 1977; Mathews \& Bregman 1978). These observations led to the development of the cooling flow $(\mathrm{CF})$ model. In this model the ICM at the centers of clusters with dense cores hydrostatically cools, so that the cool gas is compressed by the weight of the overlying gas. Hot gas from the outer regions of the ICM flows in to replace the compressed gas, generating a CF. Although early X-ray observations seemed to corroborate this

* Appendices and Tables 2 and 3 are only available in electronic form at http://www . aanda.org model and there was some evidence of expected $\mathrm{H} \alpha$ emission and UV emission, optical observations failed to detect the expected star formation rates, $\mathrm{CO}$ and molecular gas (McNamara \& O'Connell 1989; Edge 2001). Later, discrepancies between spectrally determined mass deposition rates from ASCA and the classical determination from density further brought the classical CF model into question (Makishima et al. 2001, and references therein). More recently, grating spectra from XMM (Peterson et al. 2001; Tamura et al. 2001b,a; Kaastra et al. 2001; Xu et al. 2002; Sakelliou et al. 2002; Peterson et al. 2003; Sanders et al. 2008) have revealed that the gas in central regions is not cooling at the rates predicted by the traditional cooling flow model. The classical CF model assumes that no mechanism exists to significantly heat the gas. Therefore, this discrepancy has inspired a search for heating models that can explain current 
observations. Among those mechanisms proposed are: conduction (e.g. Zakamska \& Narayan 2003), central AGN heating via direct cosmic ray-ICM interaction+conduction (Guo \& Oh 2008), AGN heating by bubble induced weak shocks (e.g. Mathews et al. 2006), soundwaves+conduction (e.g. Ruszkowski et al. 2004) and turbulence+conduction (e.g. Dennis \& Chandran 2005). On the observational side recent studies (e.g. Bîrzan et al. 2004; Rafferty et al. 2006; Dunn \& Fabian 2006, 2008; Bîrzan et al. 2008) have looked at the mechanical energy released by AGN driven bubbles and compared it to the energy needed to quench the $\mathrm{CF}$.

The failure of the classical $\mathrm{CF}$ model has changed the nomenclature of these centrally dense clusters from CF clusters to cool-core (CC) clusters as suggested by Molendi \& Pizzolato (2001). One problem with this nomenclature is that it is unclear what distinguishes a $\mathrm{CC}$ cluster from a non-CC (NCC) cluster. The name implies that gas in the center of the cluster is cool, but does that always imply a short cooling time? In fact authors define CC clusters differently often based on a central temperature drop (e.g. Sanderson et al. 2006a; Burns et al. 2008), short central cooling time (e.g. Bauer et al. 2005; O'Hara et al. 2006; Donahue 2007), or significant classical mass deposition rate (Chen et al. 2007). There also is a question as to whether there is a distinct difference between NCC and CC clusters. That is, is there a parameter that unambiguously distinguishes NCC clusters from CC clusters? This is a nontrivial question since, when used as cosmological probes, clusters are often segregated into $\mathrm{CC} / \mathrm{NCC}$ subsamples. Frequently $\mathrm{CC}$ clusters are chosen for mass determination studies since they are believed to be dynamically relaxed. On the other hand, it requires a large amount of energy to quench a $\mathrm{CF}$, which may strongly affect the entire ICM. For example, O'Hara et al. (2006) found that $\mathrm{CC}$ clusters (as defined as having a central cooling time (CCT) more than $3 \sigma$ below $7.1 h_{70}^{-1 / 2}$ Gyr) have more scatter about scaling relations than NCC clusters. Therefore it becomes important to unambiguously differentiate between CC and NCC clusters before proceeding with determining their effects on scaling relations.

It is worth noting that a discrepancy between mass deposition rates determined spectroscopically and those from images in some clusters does not indicate in itself any breakdown of the simple cooling flow picture, since it may be that these clusters just did not have enough time to deposit the predicted level of mass. Here, we will show for the first time that this recent formation solution to the cooling flow discrepancy is very unlikely, based on statistical arguments.

Recently, Chen et al. (2007) investigated the cores of a sample of 106 galaxy clusters based on the extended HIghest X-ray FLUx Galaxy Cluster Sample (HIFLUGCS) sample (Reiprich \& Böhringer 2002) using ROSAT and ASCA, with their primary goal to study the effect of CC versus NCC clusters on scaling relations. They defined a $\mathrm{CC}$ cluster as one that has significant classically defined mass deposition rate $\left(\dot{M}_{\text {classical }}\right)$ and found $49 \%$ of their sample were CC clusters. Additionally they determined that the fraction of CC clusters in their sample decreased with increasing mass. Sanderson et al. (2006a) investigated the cores of a statistically selected sample of the 20 brightest $^{1}$ HIFLUGCS clusters using Chandra. They find that nine $(\sim 41 \%)$ of their clusters are CC clusters, where they define $\mathrm{CC}$ clusters as having a significant drop in temperature in the central region. Additionally they find that the slope of the inner

\footnotetext{
1 The Coma, Fornax, and Centaurus clusters were excluded because of their large angular size.
}

temperature profiles has a bimodal distribution with NCC clusters having a flat temperature profile and $\mathrm{CC}$ clusters having a slope $(\mathrm{d} \log (T) / \mathrm{d} \log (r))$ of 0.4 . Burns et al. (2008) investigated the properties of CC and NCC clusters from a cosmological simulation which produced both types of clusters. As with Sanderson et al. (2006a), they define CC clusters based on the central temperature decrease (by $20 \%$ of $T_{\text {vir }}$ in their case). Their simulations show that an early merger determines whether a cluster is a $\mathrm{CC}$ cluster or not and suggest that $\mathrm{CC}$ clusters are not necessarily more relaxed than NCC clusters.

Additionally, studies have begun on samples of distant (high- $z$ ) clusters. The goal of these studies is to determine the physical evolution of CC clusters and whether the CC fraction changes with redshift. The major obstacle for these high- $z$ cluster studies is that the traditional indications of CCs are difficult to measure for distant clusters. Therefore authors offer proxies, based on studies of low- $z$ clusters, that can be measured for distant clusters with limited signal. Vikhlinin et al. (2007) suggested cuspiness as a proxy for determining whether a distant cluster is a CC cluster or not. Cuspiness is defined as:

$\alpha \equiv-\frac{\mathrm{d} \log (n)}{\mathrm{d} \log (r)}$ at $r=0.04 r_{500}$,

where $n$ is the electron density and $r$ is the distance from the cluster center. For their sample of 20 clusters with $z>0.5$, they find a lack of CC clusters. That is, they find no strong CC clusters $(\alpha>0.75$ by their definition) and few weak CC clusters ( $\alpha=0.5-0.75$ ). Approximately $67 \%$ of their nearby sample (a subsample of 48 HIFLUGCS clusters) has $\alpha>0.5$ versus $15 \%$ for their $z>0.5$ sample. On the other hand, using simulations, Burns et al. (2008) find that the CC fraction stays constant back to a redshift of $z \sim 1$. Recently Santos et al. (2008) compared a nearby sample of 11 clusters $(z=0.15-0.3)$ to a distant sample of 15 clusters $(z=0.7-1.4)$. They suggest a concentration parameter as a proxy for identifying $\mathrm{CC}$ clusters:

$C_{\mathrm{SB}} \equiv \frac{\Sigma(r<40 \mathrm{kpc})}{\Sigma(r<400 \mathrm{kpc})}$

where $\Sigma(r<40 \mathrm{kpc})$ and $\Sigma(r<400 \mathrm{kpc})$ are the integrated surface brightnesses within $40 \mathrm{kpc}$ and $400 \mathrm{kpc}$ respectively. As with Vikhlinin et al. (2007), they find no strong CC clusters in their high redshift sample. On the other hand they find roughly the same fraction of weak (or moderate) CC clusters in the distant as in the local sample, in apparent conflict with Vikhlinin et al. (2007). However, since both the nearby and distant samples of Santos et al. (2008) are not complete, their fractions may be biased by selection factors, so a straightforward comparison is not possible.

In this paper we investigate a statistically complete, fluxlimited sample of $64 \mathrm{X}$-ray selected clusters and analyze their cores with the Chandra ACIS instrument. This is the first detailed core analysis of a nearby, large, complete sample with a high resolution instrument. With its $\sim 0.5$ point spread function, Chandra is the ideal instrument for such a study. Likewise, the HIFLUGCS sample, which comprises the $64 \mathrm{X}$-ray brightest clusters outside the Galactic plane (Reiprich \& Böhringer 2002), is an ideal sample. Since the clusters are bright, they are nearby $(\langle z\rangle=0.053)$ making it possible to probe the very central regions of the clusters. Moreover they have high signal to noise allowing precise measurements to be made. It is worth noting that complete flux-limited samples are not necessarily unbiased or representative with respect to morphology (e.g. Reiprich 2006). The point is that, for flux-limited samples, 
Table 1. Nomenclature.

\begin{tabular}{cc}
\hline \hline Abbreviation & \\
\hline ICM & IntraCluster Medium \\
CCT & Central Cooling Time at $0.4 \% R_{500}$ \\
SCC & Strong Cool Core \\
WCC & Weak Cool Core \\
NCC & Non Cool Core \\
CC & Cool Core (SCCs+WCCs) \\
CF & Cooling Flow \\
BCG & Brightest Cluster Galaxy \\
EP & Emission Peak (X-ray) \\
BCG-EP & Projected separation between BCG and EP \\
\hline
\end{tabular}

the bias can, in principle, be calculated (e.g., Ikebe et al. 2002; Stanek et al. 2006). Also, such samples are representative in the sense that their statistical properties, like cooling core frequency, are directly comparable to the same properties of simulated fluxlimited samples. Samples constructed based on availability of data in public archives are, in general, not representative in this sense ("archive bias").

Our goal in this paper is to determine if there is a physical property that can unambiguously segregate CC from NCC clusters. We use this property to examine other parameters to see how well they may be used as proxies when determining whether a cluster is a CC cluster or not. The article is organized as follows. We outline our methods of data reduction in Sect. 2. We present our results in Sect. 3, wherein we describe the various parameters investigated for determining whether a cluster is a CC or an NCC cluster in Sect. 3.1, determine the best parameter to segregate distant CC and NCC clusters in Sect. 3.2, compare this parameter to others in Sect. 3.3 and determine the best diagnostic for cool cores in distant clusters in Sect. 3.4. We discuss our results in Sect. 4, wherein we describe the basic cooling flow problem in Sect. 4.1 and discuss the inner temperature profiles in Sect. 4.2, the central temperature drop seen in some clusters in Sect. 4.2, the relation between cluster mergers and the projected separation between the BCG and X-ray peak in Sect. 4.3 and the WCC clusters in Sect. 4.4. Finally we give our conclusions in Sect. 5. To aid clarity, we give in Table 1 some of the abbreviations used throughout the paper.

In this work, we assume a flat $\Lambda$ CDM Universe with $\Omega_{\mathrm{M}}=$ $0.3, \Omega_{\mathrm{vac}}=0.7$, and $H_{0}=h_{71} 71 \mathrm{~km} \mathrm{~s}^{-1} \mathrm{Mpc}^{-1}$. Unless otherwise noted, $k$ is the Boltzmann constant and we use the following nomenclature for our coordinates: $x$ is the projected distance from the cluster center, $r$ is the physical 3-D distance from the cluster center and $l$ is the distance along the line of sight. All errors are quoted at the $1 \sigma$ level unless otherwise noted.

\section{Observations and methods}

All 64 HIFLUGCS clusters have been observed with Chandra, representing $4.552 \mathrm{Ms}$ of cleaned data. For our analysis we used all unflared data taken after the CCD focal plane temperature was reduced to $-120{ }^{\circ} \mathrm{C}$ (2001-Jan.-29) and was publicly available as of 2007-May-07, with a few exceptions. (1) In cases where more than one data set was publicly available and one set was heavily flared, the entire flared data set was discarded; (2) for $\mathrm{A} 0754^{2}$ and $\mathrm{A} 0401^{3}$; (3) in the cases of A2597 and

\footnotetext{
2 There are later observations of A0754, but they are all far offset and not useful for core studies and therefore not included.

3 There is a post-2001-Jan.-29 observation of A401, however it is offset such that the cluster center is in the corner of a CCD. We therefore included the centered pre-2001-Jan.-29 observation as well.
}

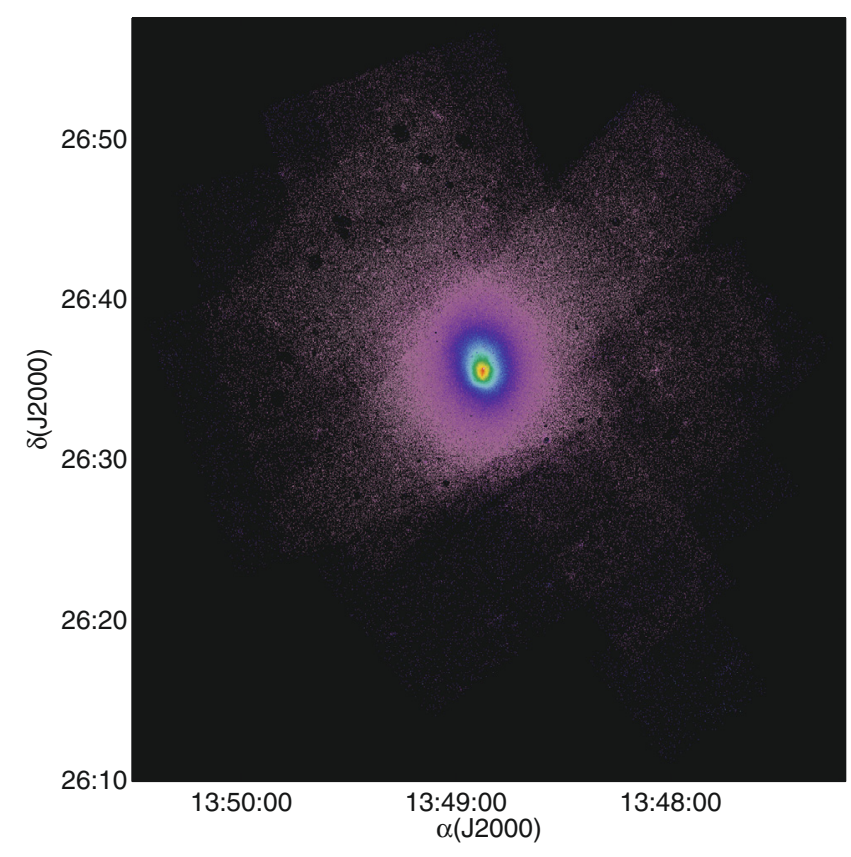

Fig. 1. An example of a mosaiced image. This is the background subtracted, exposure corrected image created from the 12 Chandra exposures of A1795.

A2589 the original observations were heavily flared and the PIs (Clarke and Buote, respectively) of the newer proprietary data sets graciously provided them to us before they became publicly available (4) the Coma Cluster (A1656), has a recently released calibration observation (2008-Mar.-23) which we used instead of the pre-2001-Jan.-29 observations. Several other PI's kindly provided data sets before they were publicly available, although they since have become publicly available (see acknowledgments). Details on the number and length of the observations can be found in Table 2. Note that we use redshifts compiled from NASA/IPAC Extragalactic Database (NED) ${ }^{4}$ and the values did not differ significantly $(\Delta z<0.001)$ from the values used by Reiprich \& Böhringer (2002).

\subsection{Data reduction}

The basic data reduction was done using CIAO 3.2.2 and CALDB 3.0 following the methods outlined in Hudson et al. (2006). Since we are interested in the cores of clusters, we took the cluster center to be the emission peak (EP) from the background subtracted, exposure corrected image. See Hudson et al. (2006) for details of our image creation technique ${ }^{5}$. To avoid statistical fluctuations in determining the cluster peak, we smoothed all images with a Gaussian. In general we used a $\sim 4$ " kernel, however in a few particularly noisy cases (noted in Appendix C) we used a larger smoothing kernel. Figure 1 shows an example of a mosaiced image.

\subsection{Temperature profiles}

Using the EP as the cluster center we created a temperature profile for each cluster using the following method. In order to get good statistics for our temperature profiles, we estimated that 10000 source counts per annulus would give us a $5 \%$

\footnotetext{
4 http://nedwww.ipac. caltech.edu

5 For this analysis we did not apply adaptive smoothing.
} 
constraint on $T$. However, in several cases we had a small number of total source counts $(\sim 30000)$, so that using 10000 source counts per annulus was not practical. We therefore extracted annuli with at least 5000 source counts for any cluster with fewer than 100000 total source counts and 10000 source counts for any cluster with more than 100000 total source counts. We created pie shaped annuli, not extending beyond the boundary of the chips. Although this decreased our efficiency, it allows for unbiased density measurements when using the normalization of the thermal model (a full circle extending beyond the chip edge would generally cover more of the inner part of the annulus than the outer). In our subsequent studies it will also allow us to use the same spectra for deprojection models as well as helping to constrain the residual cosmic X-ray background (CXB) in the outer cluster regions ${ }^{6}$. We then created spectra and fit the spectrum to an absorbed thermal model (WABS*APEC*EDGE ${ }^{7}$ ). For the majority of observations we used the radio measured hydrogen absorption columns from the Leiden/Argentine/Bonn HI-Survey (Kalberla et al. 2005) or, when available, pointed observations from E. Murphy (private communication). In a few cases, noted in Appendix $\mathrm{C}$, the $\mathrm{X}$-ray measured value was significantly higher than the radio measured value and so we left the hydrogen absorption column as a free parameter. See Baumgartner \& Mushotzky (2006) for an explanation of the discrepancy between radio and X-ray absorption column density measurements. As noted in Table 3 and Appendix C, there were 14 cases $(\sim 22 \%)$ in which we added a second thermal component to one or more of the central annuli in order to improve the fit. In these cases, the lower of the two temperatures was used for our annular analysis. There were also a few cases in which using non-solar metalicity ratios significantly improved the fit, however since the best-fit temperature was unaffected, solar ratios were used for simplicity. See Appendix C for notes on the clusters where this was the case.

\subsection{Cluster virial temperature, radius, and mass}

The cluster virial temperature $\left(T_{\text {vir }}\right)$ is the temperature of the $\mathrm{X}$-ray emitting gas that is in hydrostatic equilibrium with the cluster potential. Although $T_{\text {vir }}$ is not an imperative quantity for core studies, it is useful for scaling clusters for comparison purposes (e.g. relative temperature drop) or estimating the cluster virial radius and mass. In this section we describe our method for determining $T_{\text {vir }}$ and how we use it to estimate the virial mass and radius.

The temperature decline in the central regions of some clusters, if included, is the largest source of bias in the determination of $T_{\text {vir }}$. The reason for this temperature drop is a current topic of debate and discussed in more detail in Sect. 4.2. Here we simply discuss the removal of this central region, so it does not bias the fit to $T_{\text {vir. }}$. In order to determine the size of the central region to be excluded, we fit our temperature profiles to a broken powerlaw. The core radius in the powerlaw was free and the index of the outer component was fixed to be zero. Additionally we removed the outer annuli where accurate subtraction of the blank sky backgrounds (BSB) becomes critical and where clusters may have a decreasing temperature profile (Markevitch et al. 1998; De Grandi \& Molendi 2002; Vikhlinin et al. 2005;

\footnotetext{
${ }^{6}$ The normalizations for the cluster emission for partial annuli of different observations can be scaled and tied when trying to determine the residual CXB.

7 The edge component is a correction to underestimation of Chandra's efficiency at $\sim 2 \mathrm{keV}$ (see Vikhlinin et al. 2005).
}
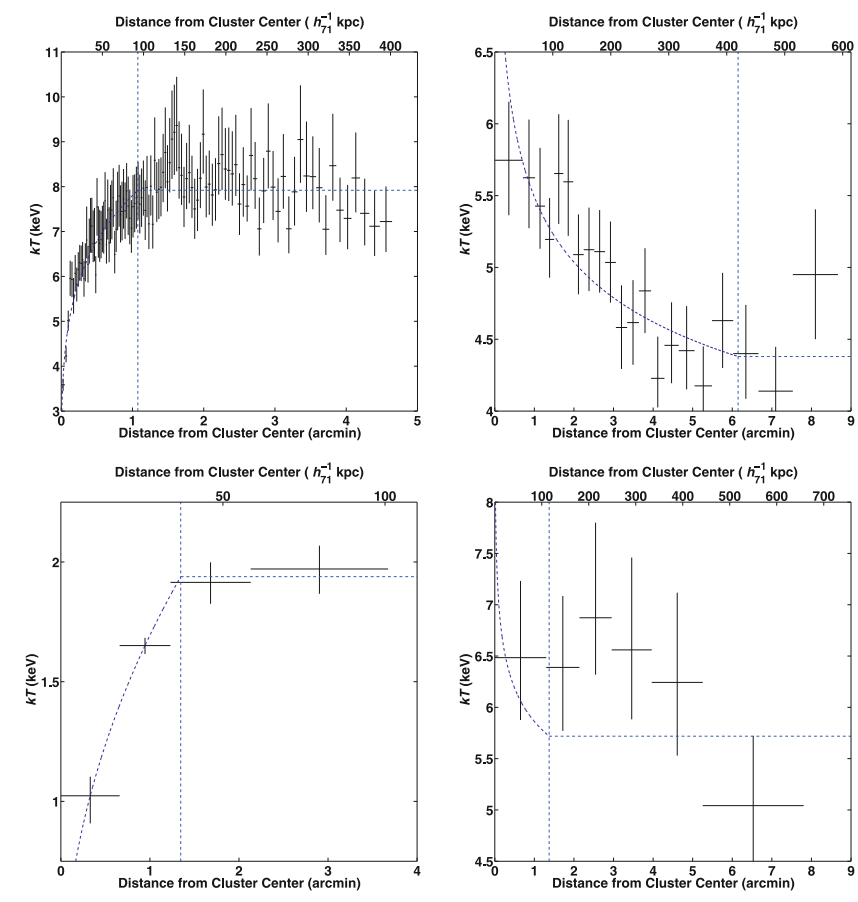

Fig. 2. These figures show the broken powerlaw fit to four typical temperature profiles representing long and short exposures of clusters with a central temperature drop and clusters without a central temperature drop. The dashed blue lines indicate the fit and the core radius. Top left: A2029 - a long exposure cluster with a central temperature drop. Top right: A3158 - a long exposure of a cluster without a central temperature drop. Bottom left: A3581 - a short exposure of a cluster with a central temperature drop. Bottom right: ZwCl1215 - a short exposure of a cluster without a central temperature drop.

Burns et al. 2008). The core radius in the powerlaw was taken to be the radius of the excluded central region. Table 2 gives this core radius as well as $k T_{\text {vir }}$ and overall cluster metalicity. Figure 2 shows examples of the broken powerlaw fit to the temperature profiles of four representative clusters: (1) a long exposure of a cluster that has a central temperature drop; (2) a long exposure of a cluster without a central temperature drop; (3) a short exposure of a cluster with a central temperature drop and (4) a short exposure of a cluster without a central temperature drop. If the core radius in the powerlaw was consistent with zero and/or the temperature gradient ( $\Gamma$ in Col. 5 of Table 3 ) was positive, no central region was removed when determining $T_{\text {vir }}$. This method allowed us to take full advantage of the largest possible signal, while being certain that $T_{\text {vir }}$ was not biased by cool central gas. Our results suggest that using a fixed fraction of the virial radius is not efficient and can be dangerous since the core radius, as defined above, does not scale with the cluster virial radius.

Since we include all data outside of the core radius when determining $T_{\text {vir }}$, there are regions where residual background becomes important. This was especially true for some of our more distant clusters (e.g. RX J1504, $z=0.2153$ ). The residual background results from three factors: (1) since the BSB is scaled to remove the particle background by matching the BSB 9.5-12 keV rate to that of the observation, if the scaling factor is much different from unity, the cosmic X-ray background $(\mathrm{CXB})$ in the BSB will be significantly increased or decreased before it is subtracted; (2) likewise, the soft CXB component varies over the sky (e.g. Kuntz 2001) so the CXB in the observation and BSB will be different and (3) in back-illuminated (BI) 
CCDs, there are sometimes residual soft flares. To compensate for these effects a second soft thermal component was included as well as, in the BI CCDs, an un-folded powerlaw (i.e. a powerlaw that is not folded through the instrument response). The temperature of the soft thermal component was not allowed to exceed $1 \mathrm{keV}$ with a frozen solar abundance and zero redshift. The normalization of the soft component was also allowed to be negative in the case of oversubtraction of the CXB (e.g. for a large scaling factor). For all CCDs of the same type (i.e. front or back illuminated) in a given observation, we tied the residual background components with the normalization scaled by area. We emphasize that for most clusters this step was taken as a precaution since the cluster emission dominated over the residual CXB. Only in the most distant clusters of our sample, where the outer regions are beyond $\sim 0.5 r_{\mathrm{vir}}$, is this correction essential. As an example the best-fit temperature for RX J1504 without this correction is 7.1-7.9 keV versus $8.4-10.9 \mathrm{keV}$ with the residual BSB correction. On the other hand for A0085 $(z=0.055)$ no significant difference in temperature is found with or without the correction.

Once we measured $T_{\text {vir }}$, we used it to determine a characteristic cluster radius and mass. In the case of radius we used the scaling relation determined by Evrard et al. (1996):

$\left(\frac{r_{j}}{h_{71}^{-1} \mathrm{Mpc}}\right)=N_{j}\left(\frac{k T_{\mathrm{vir}}}{10 \mathrm{keV}}\right)^{\frac{1}{2}}$,

where $j$ is the average overdensity, within $r_{j}$, above the critical density $\left(\rho_{\mathrm{c}} \equiv 3 H_{0}^{2} / 8 \pi G\right)$ desired and $N_{j}$ is the normalization. We use normalizations $N_{500}=2.00$ and $N_{180}=2.75$, and define $r_{\text {vir }} \equiv r_{180}$. We note that these values may be overestimates of $r_{500}$ and $r_{180}$ since they are based on early simulations (e.g. Finoguenov et al. 2001). For the purposes of core studies, however, this approximation should provide an adequate approximation of a characteristic cluster radius.

To estimate a characteristic cluster mass $\left(M_{500}\right)$, we used the formulation of Finoguenov et al. (2001) derived from the ROSAT and ASCA observations of the HIFLUGCS clusters,

$\left(\frac{M_{500}}{10^{13} h_{71}^{-1} M_{\odot}}\right)=(2.5 \pm 0.2)\left(\frac{k T_{\text {vir }}}{1 \mathrm{keV}}\right)^{1.676 \pm 0.054}$.

\subsection{Central temperature drops}

To quantify the central temperature drop we calculated two quantities shown in Cols. 4 and 5 of Table 3. One is simply the temperature of the central annulus $\left(T_{0}\right)$ divided by the virial temperature $\left(T_{\text {vir }}\right)$. The second is the slope of the powerlaw fit to the central temperature profile. For clusters with a measured central temperature drop (Sect. 2.3), we fit the slope out to the break in the powerlaw. For those clusters with no central temperature drop, we fit the three innermost annuli ${ }^{8}$. The purposes of this measurement was (1) to check for a universal central temperature profile shape and (2) differentiate between a true systematic drop and random temperature fluctuations seen in merging clusters (e.g. A0754).

\subsection{Density profiles}

As with the temperature profile, the surface brightness profile becomes uncertain at large radii due to uncertainties in the residual

\footnotetext{
8 In a few cases there were clusters with central drops that did not have three bins out to the core radius. In these cases we also fit the powerlaw to the inner three annuli.
}

background. Since we are only interested in the central regions and the surface brightness falls off rapidly at large radii, we focused on fitting the central regions only. We argue that any uncertainty in the shape of the profile in the outer regions has a negligible effect when deprojecting the central regions. Since one of the parameters we are interested in, cuspiness (see Sect. 2.9 and Eq. (1)), is defined in terms of the derivative of the log of the density profile at $0.04 r_{500}$, we decided to use a slightly larger (20\%) region for determining the density profiles. This way we would have a constraint on the derivative of the log of the density profile at $0.04 r_{500}$. Specifically, we extracted a spectrum from the projected central region $\left(0-0.048 r_{500}\right)$ and fit it with an absorbed thermal model (WABS*APEC*EDGE). We used the best-fit parameters of this model to create a spectrumfile ${ }^{9}$, which we used in turn to create a weighted exposure map ${ }^{10}$.

We then created a background subtracted, exposure corrected image, in the $0.5-7.0 \mathrm{keV}$ range, similar to the method described in Hudson et al. (2006). The difference in our method here and that described in Hudson et al. (2006) is that: (1) we only used a single weighted exposure map instead of many monochromatic exposure maps for different energy bands; (2) we created an error image $\left(\sigma_{\text {img }}\right)$ and (3) we created a background subtracted image with no exposure correction. The error image was created assuming the errors in the observation, background and readout artifact or out-of-time events (OOTs) were Poisson $(\sqrt{N})$ and could be added in quadrature.

We used the background subtracted image with no exposure correction to determine annuli with at least 500 source counts ${ }^{11}$ per annulus and extracted a surface brightness profile and errors from the background subtracted exposure corrected image and the error image respectively. The surface brightness profile was then fit to a single $\beta$ model:

$\Sigma=\Sigma_{0}\left[1+\left(\frac{x}{x_{\mathrm{c}}}\right)^{2}\right]^{-3 \beta+1 / 2}$

or a double $\beta$ model:

$\Sigma=\Sigma_{01}\left[1+\left(\frac{x}{x_{\mathrm{c}_{1}}}\right)^{2}\right]^{-3 \beta_{1}+1 / 2}+\Sigma_{02}\left[1+\left(\frac{x}{x_{\mathrm{c}_{2}}}\right)^{2}\right]^{-3 \beta_{2}+1 / 2}$

(Cavaliere \& Fusco-Femiano 1976), where $x_{\mathrm{c}}$ is the core radius, $\Sigma_{0}\left(\Sigma_{0}=\Sigma_{01}+\Sigma_{02}\right.$ for a double $\beta$ model $)$ is the central surface brightness and $\beta$ is constrained to be less than 2 .

From $\beta$ and $x_{\mathrm{c}}$ for a fit to a $\beta$ model $\left(\beta_{1}, \beta_{2}, x_{\mathrm{c}_{1}}\right.$ and $x_{\mathrm{c}_{2}}$ for a fit to a double $\beta$ model), we extracted the shape of the density profile.

$n=n_{0}\left[1+\left(\frac{r}{r_{\mathrm{c}}}\right)^{2}\right]^{-\frac{3 \beta}{2}}$

and

$n=\left(n_{01}^{2}\left[1+\left(\frac{r}{r_{\mathrm{c}_{1}}}\right)^{2}\right]^{-3 \beta_{1}}+n_{02}^{2}\left[1+\left(\frac{r}{r_{\mathrm{c}_{2}}}\right)^{2}\right]^{-3 \beta_{2}}\right)^{\frac{1}{2}}$

\footnotetext{
${ }^{9}$ See http://cxc.harvard.edu/ciao/ahelp/mkinstmap.html for the definition of a spectrumfile.

${ }^{10}$ See http://space.mit.edu/CXC/docs/expmap_intro.ps.gz for more details on this procedure.

${ }^{11}$ In a few cases, if the $\beta$ model fit was poor, this was reduced to $>100$ counts.
} 
for a single and double $\beta$ model respectively, where $n_{0}$ is the central electron density, $r_{\mathrm{c}}$ is the physical core radius associated with $x_{\mathrm{c}}$ for our cosmology and $n_{0}=\sqrt{n_{01}^{2}+n_{02}^{2}}$. In order to calculate the normalization $\left(n_{0}\right)$ of the model, we used the best-fit spectral parameters from the $0-0.048 r_{500}$ regions, as described below.

For a single $\beta$ model the central electron density, $n_{0}$ is:

$n_{0}=\left(\frac{10^{14} 4 \pi D_{\mathrm{A}} D_{\mathrm{L}} \zeta \mathcal{N}}{\mathrm{EI}}\right)^{\frac{1}{2}}$,

where $\mathcal{N}$ is the normalization of the APEC model, $D_{\mathrm{A}}$ is the angular diameter distance, $D_{\mathrm{L}}$ is the luminosity distance, $\zeta$ is the ratio of electrons to protons $(\sim 1.2)$ and EI is the emission integral divided by the central density:

$\mathrm{EI} \equiv \int\left(\frac{n}{n_{0}}\right)^{2} \mathrm{~d} V$.

Inserting Eqs. (7) into (10), we get:

$\mathrm{EI}=2 \pi \int_{-\infty}^{\infty} \int_{0}^{R} x\left(1+\frac{x^{2}+l^{2}}{x_{\mathrm{c}}^{2}}\right)^{-3 \beta} \mathrm{d} x \mathrm{~d} l$

for the $0-0.048 r_{500}$ region fitted with the APEC model (Mewe et al. 1985, 1986; Smith \& Brickhouse 2000; Smith et al. 2001) and $R=0.048 r_{500}$.

For a double $\beta$ model the central electron density, $n_{0}$ is:

$n_{0}=\left[\frac{10^{14} 4 \pi\left(\Sigma_{12} \mathrm{LI}_{2}+\mathrm{LI}_{1}\right) D_{\mathrm{A}} D_{\mathrm{L}} \zeta \mathcal{N}}{\Sigma_{12} \mathrm{LI}_{2} \mathrm{EI}_{1}+\mathrm{LI}_{1} \mathrm{EI}_{2}}\right]^{\frac{1}{2}}$,

with the same definition of variables as Eq. (9). Additionally, $\Sigma_{12}$ is the ratio of the central surface brightness of model-1 to model-2, $\mathrm{EI}_{i}$ is the emission integral for model- $i$ as defined by Eq. (11), and $\mathrm{LI}_{i}$ is the line emission measure for model- $i$, defined as,

$\mathrm{LI}_{i} \equiv \int_{-\infty}^{\infty}\left(1+\frac{l^{2}}{x_{\mathrm{c}_{\mathrm{i}}}^{2}}\right)^{-3 \beta_{i}} \mathrm{~d} l$.

See Appendix A for details on this calculation.

\subsection{Cooling times}

Once we created the density profiles, we used them, together with the cooling function of the best-fit temperature, to estimate the average cooling time of the gas using:

$t_{\text {cool }}=\frac{3}{2} \frac{\left(n_{\mathrm{e}}+n_{\mathrm{i}}\right) k T}{n_{\mathrm{e}} n_{\mathrm{H}} \Lambda(T, Z)}$,

where $n_{\mathrm{i}}$ and $n_{\mathrm{e}}$ are the number density of ions and electrons respectively. The cooling function, $\Lambda(T, Z)$, was calculated by spline interpolation on a table of values for the APEC model, for an optically thin thermal plasma, kindly provided by Smith. We define the central cooling time (CCT) as:

$t_{\text {cool }}(0)=\frac{3}{2} \zeta \frac{\left(n_{\mathrm{e} 0}+n_{\mathrm{i} 0}\right) k T_{48}}{n_{\mathrm{e} 0}^{2} \Lambda\left(T_{48}\right)}$,

where $n_{\mathrm{e} 0}$ and $n_{\mathrm{i} 0}$ are the central electron and ion densities, respectively, and $T_{48}$ is the average temperature of the $0-0.048 r_{500}$ region.
We note here that in order to remove the bias introduced by different physical resolutions due to different cluster distances, we took any parameter calculated at $r=0$, (e.g. $n_{0}$ and CCT) to be the value at $r_{0} \equiv 0.004 r_{500}$. The exception to this is $T_{0}$, which is defined as the average temperature of the central annulus, which in all cases had a radius $<0.004 r_{500}$. We define $T_{\text {ctr }}$ to be the central temperature of the cluster, either $T_{48}$ or $T_{0}$ depending on the parameter considered. In general $T_{48} \approx T_{0}$ $\left(\left\langle T_{0} / T_{48}\right\rangle=0.83\right)$.

\subsection{Entropy}

As is typically done in X-ray studies of galaxy clusters, we define entropy as $K \equiv k T n^{-2 / 3}$ and central entropy as $K_{0}=$ $k T_{0} n_{0}^{-2 / 3}$, where $n_{0}$ is the central electron density and $T_{0}$ is the temperature of the innermost annulus from the temperature profile. Additionally we define a purposely biased entropy ( $\left.K_{\mathrm{BIAS}}\right)$ that takes advantage of the inherent difference in the density distribution of CC and NCC clusters to further separate them. Specifically, taking into account the fact that $\mathrm{CC}$ clusters should have higher density cores, they will, in general, have a smaller central annulus (in the temperature profile, see Sect. 2.2) than NCC clusters (when fitting spectra). That is, the 10000 (or 5000) source count threshold for the central annulus will be reached at a smaller radius for CC clusters than NCC clusters, as the density is higher and typically the temperature is lower in this region for $\mathrm{CC}$ clusters than for NCC clusters. We then define the density as the average density for the innermost annulus,

$n_{\text {annulus }}=\left(\frac{3 \times 10^{14} D_{\mathrm{A}} D_{\mathrm{L}} \zeta \mathcal{N}}{r_{\text {annulus }}^{3}}\right)^{\frac{1}{2}}$,

where the variables are defined as before and $r_{\text {annulus }}$ is the physical radius of the central annulus. Since the central annulus for clusters with a central peak in density will be smaller, its value of $n_{\text {annulus }}$ will be closer to $n_{0}$ than for clusters without a centrally peaked density. Unfortunately, since we define $r_{\text {annulus }}$ by a constant number of source counts there are additional biases due to length of the observation and redshift of the cluster. We argue, however that central density is a stronger effect. As an example we give the comparison of A2204 and A3158. A2204 has a redshift of $z=0.1522$ with a good observation time of $\sim 18.6 \mathrm{ks}$. A3158 - a cluster without a bright core, has a redshift of $z=0.06$ and good observing time of $\sim 55.7 \mathrm{ks}$. The innermost annulus ${ }^{12}$ of A2204 has a radius of 7. . $0=18.3 h_{71}^{-1} \mathrm{kpc}$, smaller than the innermost annulus of A3158 which has a radius of $42^{\prime \prime} 9=48.8 h_{71}^{-1} \mathrm{kpc}$. Even in the extreme case of A3667 $(z=0.0566)$ that has a good observing time $(\sim 485.3 \mathrm{ks})$ more than 20 times longer than A2204, the innermost annulus has a radius $\left(r_{\text {annulus }}=15^{\prime \prime} .9=17.0 h_{71}^{-1} \mathrm{kpc}\right.$ ) comparable to A2204. Based on this intentional bias, we define $K_{\mathrm{BIAS}}=k T_{0} n_{\text {annulus }}^{-2 / 3}$, where as with $K_{0}, T_{0}$ is the temperature of the central annulus from the temperature profile.

\subsection{Mass deposition rates}

We define three mass deposition rates: the classically determined mass deposition rate $\left(\dot{M}_{\text {classical }}\right)$, the spectrally determined mass deposition rate $\left(\dot{M}_{\text {spec }}\right)$ and the modified spectrally determined mass deposition rate $\left(\dot{M}_{\text {spec } 2}\right) . \dot{M}_{\text {classical }}$ is calculated from the gas

12 For comparison purposes this is the radius for 10000 counts. However when making the temperature profile for A2204 we used 5000 count annuli. 
density and temperature assuming no energy input. Using the formulation of Fabian \& Nulsen (1979), within radius $r$,

$\dot{M}_{\text {classical }}(<r) \simeq \frac{M_{\text {gas }}(r)}{t_{\text {cool }}(r)-t_{\text {cool }}(0)}$.

Usually $\dot{M}_{\text {classical }}$ is calculated at a certain radius $r_{\text {cool }}$, where we have chosen $r_{\text {cool }}$ as the radius at which $t_{\text {cool }}=7.7 h_{71}^{-1 / 2}$ Gyr. We chose $7.7 \mathrm{Gyr}$, since it corresponds to the look back time of $z=1$, a representative period over which a cluster has time to relax and form a cooling flow (Rafferty et al. 2006). However, for uniformity in comparison with our spectral mass deposition rates, we took $r$ to be the smaller of $0.048 r_{500}$ and $r_{\text {cool }}$. Furthermore, to check the effect of this cut, we extrapolated our density profiles to calculate the classical mass deposition rates out to the cooling radius, assuming the average gas characteristics remain the same and the slope of the profile remains at our fit value. The mean cooling radius was $\sim 0.07 r_{500}$, and the mass deposition rates at the cooling radius were indistinguishable, within the errors in $\dot{M}_{\text {classical }}$, from those calculated at $0.048 r_{500}$.

$\dot{M}_{\text {spec }}$ is a direct measurement of the amount of gas that is cooling by fitting the expected line emission (e.g. Peterson et al. 2003) from the multiphase gas. In order to determine $\dot{M}_{\text {spec }}$, we fit the $0-0.048 r_{500}$ region to an absorbed thermal model with a cooling flow model (Mushotzky \& Szymkowiak 1988) (WABS*[APEC+MKCFLOW]*EDGE). The higher temperature component of the cooling flow model was tied to the thermal model and the lower temperature component was frozen at $0.08 \mathrm{keV}$. Given the moderate spectral resolution of the Chandra ACIS, we checked the reliability of the measured values. Figure 3 shows the comparison between the values obtained with the XMM RGS and the Chandra ACIS for the nine clusters in common with our sample and the 14 clusters reported by Peterson et al. (2003). Figure 3 shows that the values obtained with the Chandra ACIS are, in all but one case ${ }^{13}$, consistent with the upper limits obtained by Peterson et al. (2003). Based on this we argue that the values obtained with the Chandra ACIS are reliable to well within an order of magnitude for CC clusters. As we discuss later, this value may not be accurate for NCC clusters.

$\dot{M}_{\text {spec2 }}$ is determined similarly to $\dot{M}_{\text {spec }}$, except that the lower temperature component is left as a free parameter. $\dot{M}_{\text {spec2 }}$ represents the spectral fit to a cooling flow model that is stopped at a lower-limit temperature $T_{\text {low }}$. The interpretation of this model is discussed in more detail in Sect. 4.1.

\subsection{Cuspiness}

Vikhlinin et al. (2007) suggested cuspiness as a proxy for identifying cool-core clusters at large redshift. Equation (1) gives the definition of cuspiness. For our low redshift sample, we can test the correlation of cuspiness with the parameters used to define a cool core. Since our density profile is based on a model, we define it in terms of the model parameters, with $r=0.04 r_{500}$. For a single $\beta$ model

$\alpha=\frac{3 \beta r^{2}}{\left(r_{c}^{2}+r^{2}\right)}$.

For a double $\beta$ model

$\alpha=3 r^{2} \frac{\Sigma_{12} \mathrm{LI}_{2} \beta_{1} r_{\mathrm{c}_{1}}^{-2} b_{1}^{\prime}+L I_{1} \beta_{2} r_{\mathrm{c}_{2}}^{-2} b_{2}^{\prime}}{\Sigma_{12} \mathrm{LI}_{2} b_{1}+\mathrm{LI}_{1} b_{2}}$,

13 In the case of A2052, the Chandra ACIS value is $~ 30 \%$ higher (although only a difference of $3 M_{\odot} \mathrm{yr}^{-1}$ ) than the upper limit obtained with the XMM RGS.

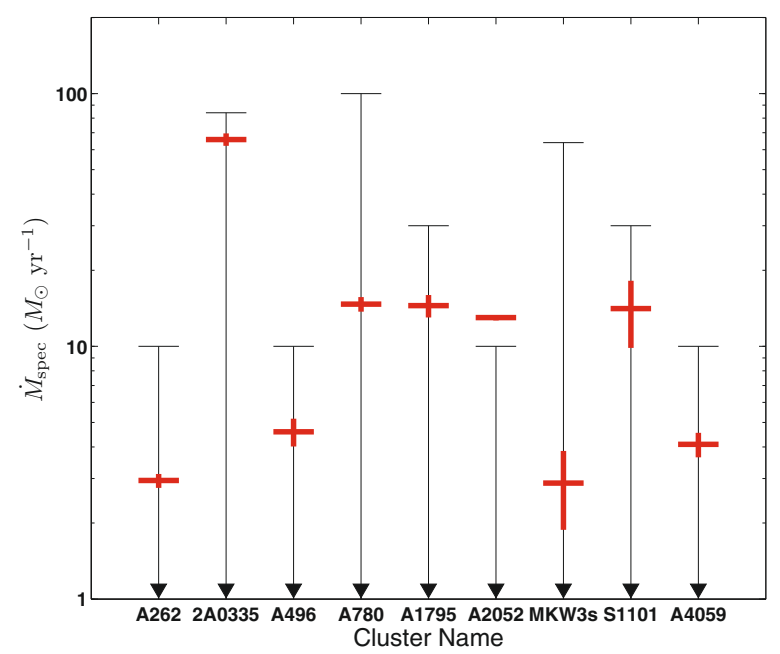

Fig. 3. XMM RGS - Chandra ACIS spectroscopic cooling rates. Here we plot the upper limits measured by Peterson et al. (2003) with the XMM RGS (black), compared to our Chandra ACIS measured values (thick red) for the nine clusters in common. For eight of the nine clusters, the Chandra ACIS gives results consistent with the XMM RGS. For the one cluster (A2052) for which they are not consistent, the value measured with the Chandra ACIS is $\sim 30 \%$ higher $\left(3 M_{\odot} \mathrm{yr}^{-1}\right)$ than the XMM RGS upper limit. This comparison corroborates the Chandra ACIS spectrally determined mass deposition rates.

where $\Sigma_{12}, \mathrm{LI}_{i}$ are defined as before and $b_{i} \equiv\left(1+\left(\frac{r}{r_{\mathrm{c}_{\mathrm{i}}}}\right)^{2}\right)^{-3 \beta_{i}}$ and $b_{i}^{\prime} \equiv\left(1+\left(\frac{r}{r_{\mathrm{c}_{\mathrm{i}}}}\right)^{2}\right)^{-3 \beta_{i}-1}$

\section{Results}

\subsection{Bimodality and histograms}

In order to determine the best parameter to separate CC clusters from NCC clusters, we tested 16 parameters for bimodality (and trimodality in some cases) using the Kaye's Mixture Model (KMM) algorithm (e.g. Ashman et al. 1994). For each parameter we used a single covariance for both (all three) subgroups when determining the significance of the rejection of the single Gaussian hypothesis. We chose to do this because analytic errors are only statistically meaningful when the same covariance is used for each subgroup. However, since there is no reason to believe the bimodality in any parameter is symmetric, we considered independent covariances for the subgroups when determining the subgroup assignments. Moreover the analytic errors when using different covariances for each subgroup can still give a good guideline on fit improvement (e.g. Ashman et al. 1994), even if their true significance is unknown.

Figure 4 shows histograms of the 16 parameters with Gaussians (created from the KMM algorithm results) overplotted. For each parameter the CC and NCC subgroups were independently determined. We constructed Gaussians using the means and covariances returned by the KMM algorithm and calculated the normalization of the Gaussians so that the integral of the Gaussian was equal to the area of the bins (sum of the number of clusters in each bin times the width of the bin) within its relevant subgroup. Bins of clusters from the CC subgroup are colored blue and bins of clusters from the NCC subgroup are colored red. In the cases with a third subgroup between $\mathrm{CC}$ and NCC clusters, the bins are colored black. 

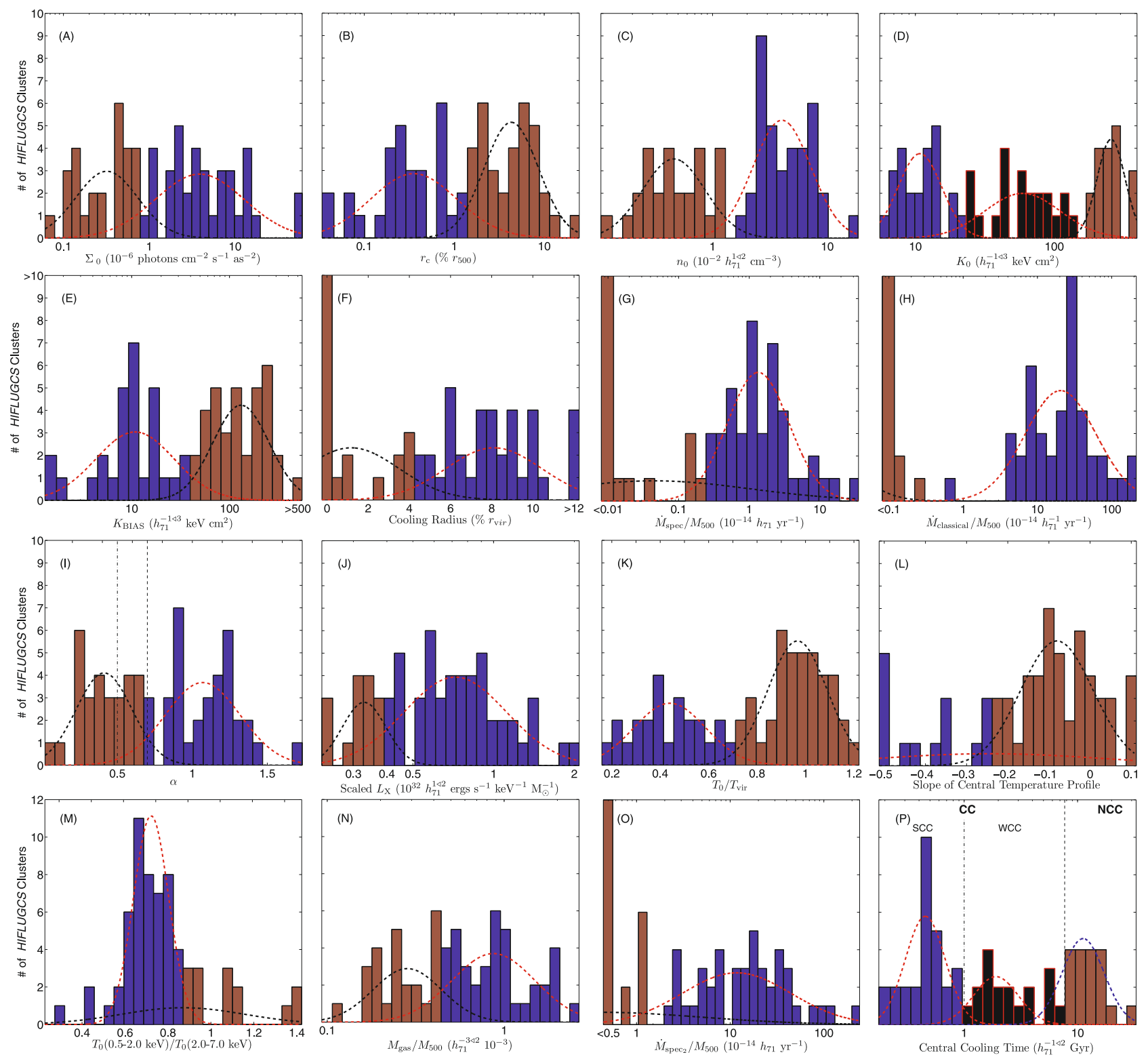

Fig. 4. Histograms of 16 parameters that may be used to distinguish between CC and NCC clusters. Blue bins represent CC clusters, red bins indicate NCC clusters and where they appear, black bins represent transitional or weak CC clusters (see text). Row-wise left to right and starting from the top row the histograms are: A) central surface brightness $\left(\Sigma_{0}\right)$; B) $\beta$ model core radius scaled by $r_{500}$; C) central electron density $\left(n_{0}\right)$; D) central entropy $\left(K_{0}\right)$; E) biased central entropy $\left(K_{\text {BIAS }}\right)$; F) cooling radius; G) spectral mass deposition rate scaled by $M_{500}\left(\dot{M}_{\text {spec }} / M_{500}\right)$; H) classical mass deposition rate scaled by $M_{500}\left(\dot{M}_{\text {classical }} / M_{500}\right)$; I) cuspiness $(\alpha)$; J) bolometric X-ray luminosity of the $0-0.048 r_{500}$ region scaled by the gas mass of the region and virial temperature $\left.\left(L_{X} /\left(M_{\mathrm{gas}} k T_{\mathrm{vir}}\right)\right) ; \mathbf{K}\right)$ central temperature drop $\left.\left(T_{0} / T_{\mathrm{vir}}\right) ; \mathbf{L}\right)$ slope of inner temperature profile; M) central temperature measured in the soft band divided by central temperature measured in the hard band; N) gas mass within $0.048 r_{500}$ scaled by $\left.M_{500} ; \mathbf{O}\right)$ modified spectral mass deposition rate scaled by $M_{500}\left(\dot{M}_{\text {spec } 2} / M_{500}\right)$ and P) central cooling time (CCT). For the cuspiness histogram, the dash-dot and dashed vertical line indicates the CC/NCC cut and the weak CC, strong CC cut, respectively, suggested by Vikhlinin et al. (2007).

\subsubsection{Central surface brightness $(A): \Sigma_{0}$}

The histogram for $\Sigma_{0}$ appears to have two peaks on either side of $\sim 10^{-6}$ photons $\mathrm{cm}^{-2} \mathrm{~s}^{-1} \operatorname{arcsec}^{-2}(0.5-7.0 \mathrm{keV})$. They are, however, not well separated and the KMM-algorithm does not reject a single Gaussian hypothesis for this data set $(<85 \%$ confidence). If divided into two Gaussians, the KMM algorithm finds a partition at $\sim 0.80 \times 10^{-6}$ photons $\mathrm{cm}^{-2} \mathrm{~s}^{-1} \mathrm{arcsec}^{-2}$, which divides that sample into $37 \mathrm{CC}$ clusters and 27 NCC clusters. The centers of the subgroups are $3.88 \times 10^{-6}$ photons $\mathrm{cm}^{-2} \mathrm{~s}^{-1} \operatorname{arcsec}^{-2}$ and $0.32 \times 10^{-6}$ photons $\mathrm{cm}^{-2} \mathrm{~s}^{-1} \operatorname{arcsec}^{-2}$ for the $\mathrm{CC}$ and $\mathrm{NCC}$ clusters respectively.

\subsection{2. $\beta$ model core radius $(\mathrm{B}): r_{\mathrm{c}}\left(\% r_{500}\right)$}

In the case of a double $\beta$ model the inner core radius was used. The histogram of $r_{\mathrm{c}}$ as a percentage of $r_{500}$ shows little evidence of bimodality, with four distinct peaks, however the KMM algorithm rejects the single Gaussian hypothesis at $\sim 99 \%$ confidence. Using the KMM algorithm we find a split at $\sim 1.3 \times 10^{-2} r_{500}$, which divides the sample into 30 CC clusters and 34 NCC clusters. The centers of the subgroups are $0.35 \times 10^{-2} r_{500}$ and $4.31 \times 10^{-2} r_{500}$ for the CC and NCC clusters respectively. 


\subsubsection{Central electron density (C): $n_{0}$}

The histogram for $n_{0}$ appears to have a single peak at $\sim 2.5 \times$ $10^{-2} h_{71}^{1 / 2} \mathrm{~cm}^{-3}$, with a lower, wider distribution below $\sim 1 \times$ $10^{-2} h_{71}^{1 / 2} \mathrm{~cm}^{-3}$. The KMM algorithm rejects the single Gaussian hypothesis at $>99 \%$ confidence. We separate the two distributions at $\sim 1.5 \times 10^{-2} h_{71}^{1 / 2} \mathrm{~cm}^{-3}$ which divides the sample into $37 \mathrm{CC}$ clusters and 27 NCC clusters. The centers of the subgroups are $4.02 \times 10^{-2} h_{71}^{1 / 2} \mathrm{~cm}^{-3}$ and $0.46 \times 10^{-2} h_{71}^{1 / 2} \mathrm{~cm}^{-3}$ for the $\mathrm{CC}$ and NCC clusters respectively.

\subsubsection{Central entropy (D): $K_{0}$}

The histogram for $K_{0}$ looks like it may have a tri-modal distribution with peaks at $\sim 10 h_{71}^{-1 / 3} \mathrm{keV} \mathrm{cm}^{2}, \sim 40 h_{71}^{-1 / 3} \mathrm{keV} \mathrm{cm}^{2}$ and $\sim 250 h_{71}^{-1 / 3} \mathrm{keV} \mathrm{cm}^{2}$. The KMM algorithm rejects the single Gaussian hypothesis at more than $99 \%$ confidence and the likelihood ratio test statistic (LRTS) (Ashman et al. 1994) suggests $K_{0}$ is one of the most significant bimodal distributions. The KMM algorithm finds a division between the two subgroups at $\sim 25 h_{71}^{-1 / 3} \mathrm{keV} \mathrm{cm}^{2}$, which divides the sample into $27 \mathrm{CC}$ and 37 NCC clusters. The division in $K_{0}$ was first reported by Hudson \& Reiprich (2007) and Reiprich \& Hudson (2007) and fits well with the results of Voit et al. (2008) and Cavagnolo et al. (2009). Adding a third subgroup, improves the LRTS further and divides the distribution at $\sim 22$ and $\sim 150 h_{71}^{-1 / 3} \mathrm{keV} \mathrm{cm}^{2}$, which gives 24 strong CC (SCC) clusters, 22 weak CC or transition (WCC) clusters and 18 NCC clusters. The centers of the subgroups are $11 h_{71}^{-1 / 3} \mathrm{keV} \mathrm{cm}^{2}, 59 h_{71}^{-1 / 3} \mathrm{keV} \mathrm{cm}^{2}$ and $257 h_{71}^{-1 / 3} \mathrm{keV} \mathrm{cm}^{2}$ for the SCC, WCC and NCC clusters respectively.

\subsubsection{Biased central entropy (E): $K_{\mathrm{BIAS}}$}

Naïvely one would expect $n_{\text {annulus }}<n_{0}$ (see Eq. (16) for the definition of $n_{\text {annulus }}$ ), however since $n_{\text {annulus }}$ is not deprojected and $n_{0}$ is deprojected, it is possible that $n_{\text {annulus }}$ will be larger than $n_{0}$, implying an appreciable amount of emission along the line of sight. This emission increases the apparent central density if it is not subtracted before determining the central density. In general, however, $n_{\text {annulus }}$ will be smaller than $n_{0}$, especially for NCC clusters which require a large region to obtain our count criterion. The effect of this intentional bias is that NCC clusters end up with a large value of $K_{\mathrm{BIAS}}$ (as can be seen, the maximum value of $K_{\mathrm{BIAS}}$ is larger than the maximum value of $K_{0}$ ). On the other hand CC clusters will have a value of $K_{\mathrm{BIAS}}$ similar to $K_{0}$, separating the distribution. The net effect of the bias seems to be to shift the 15 transition clusters in the $K_{0}$ distribution toward the NCC peak. The KMM algorithm rejects the single Gaussian hypothesis at $>99 \%$ confidence. Surprisingly the LRTS suggests the bimodal distribution for $K_{\mathrm{BIAS}}$ is less significant than for $K_{0}$. The two CC/NCC subgroups are divided at $40 h_{71}^{-1 / 3} \mathrm{keV} \mathrm{cm}^{2}$, which gives $29 \mathrm{CC}$ clusters and 35 NCC clusters. The centers of the subgroups are $11 h_{71}^{-1 / 3} \mathrm{keV} \mathrm{cm}^{2}$ and $131 h_{71}^{-1 / 3} \mathrm{keV} \mathrm{cm}^{2}$ for the $\mathrm{CC}$ and NCC clusters respectively.

\subsubsection{Cooling radius $\left(\% r_{\text {vir }}\right)(\mathrm{F})$}

We made two assumptions when calculating the cooling radius: (1) $t_{\text {cool }}\left(r_{\text {cool }}\right)=7.7 h_{71}^{-1 / 2}$ Gyr (see Sect. 2.8) and (2) the gas at the cooling radius has the density extrapolated from the $0-0.048 r_{500}$ density profile. Therefore, any cluster with a CCT longer than $7.7 h_{71}^{-1 / 2} \mathrm{Gyr}$, has a cooling radius of zero. Beyond that, there is a broad distribution above about $0.02 r_{500}$. The KMM algorithm rejects the single Gaussian hypothesis at $98 \%$ confidence, however the algorithm does not converge if different covariances are used for each subgroup (possibly because of the large peak at zero). Using a common covariance for the two subgroups, the KMM algorithm partitions the two subgroups at $0.043 r_{500}$ which divides the sample into $37 \mathrm{CC}$ clusters and $27 \mathrm{NCC}$ clusters. The centers of the subgroups are $0.081 r_{500}$ and $0.012 r_{500}$ for the $\mathrm{CC}$ and $\mathrm{NCC}$ clusters respectively. We note that since a cluster with $t_{\text {cool }}(0)>7.7 h_{71}^{-1 / 2}$ will, by definition, have cooling radius of zero, it makes a poor parameter for comparison studies.

\subsubsection{Scaled spectral mass deposition rate (G): $\dot{M}_{\text {spec }} / M_{500}$}

The histogram for $\dot{M}_{\text {spec }} / M_{500}$ shows strong evidence of a bimodal distribution. There are 13 clusters with a best-fit value of $\dot{M}_{\text {spec }}=0$ and there is a second peak at $\sim 10^{-14} h_{71} \mathrm{yr}^{-1}$. Since the KMM algorithm was fitted to the log value of the data, the clusters with $\dot{M}_{\text {spec }}=0$, were assigned to $\log \left[\dot{M}_{\text {spec }} /\left(M_{500} 10^{-14} h_{71} \mathrm{yr}^{-1}\right)\right]=-5$. The KMM algorithm rejects the single Gaussian hypothesis at $>99 \%$ confidence. The LRTS confirms that the bimodality is one of the most significant of the 16 parameters. The KMM algorithm identifies 23 clusters with the first (NCC) subgroup. However, due to the large variance in the first subgroup, the two clusters with the largest value of $\dot{M}_{\text {spec }} / M_{500}$ are assigned to the first subgroup (see Fig. 4G). Since this is physically unreasonable, we take the partition value to be $\sim 0.23 \times 10^{-14} h_{71} \mathrm{yr}^{-1}$, which divides the sample into 43 CC clusters and 21 NCC clusters. The centers of the subgroups are $1.3 \times 10^{-14} h_{71} \mathrm{yr}^{-1}$ and $0.045 \times 10^{-14} h_{71} \mathrm{yr}^{-1}$ for the $\mathrm{CC}$ and NCC clusters respectively. As with cooling radius, the existence of zero values makes $\dot{M}_{\text {spec }} / M_{500}$ problematic as a parameter for comparison studies.

\subsubsection{Scaled classical mass deposition rate $(H)$ : $\dot{M}_{\text {classical }} / M_{500}$}

As with cooling radius, the 18 clusters with CCT $>7.7 h_{71}^{-1 / 2} \mathrm{Gyr}$, have $\dot{M}_{\text {classical }}=0$. Similar to $\dot{M}_{\text {spec }} / M_{500}$, those clusters were assigned $\log \left[\dot{M}_{\text {classical }} /\left(M_{500} 10^{-14} h_{71} \mathrm{yr}^{-1}\right)\right]=-5$ when applying the KMM algorithm to the log of the data. Besides the peak at zero, there seems to be a second Gaussian distribution at $\sim 20 \times 10^{-14} h_{71} \mathrm{yr}^{-1}$. The KMM algorithm rejects the single Gaussian hypothesis at more than $99 \%$ confidence. The LRTS identifies $\dot{M}_{\text {classical }} / M_{500}$ as having the most significant bimodality. The partition between the two subgroups is $\sim 0.5 \times 10^{-14} h_{71} \mathrm{yr}^{-1}$, which divides the sample into $43 \mathrm{CC}$ clusters and 21 NCC clusters. The centers of the subgroups are $20.5 \times 10^{-14} h_{71} \mathrm{yr}^{-1}$ and $0.0106 \times 10^{-14} h_{71} \mathrm{yr}^{-1}$ for the CC and NCC clusters respectively. We note, as with cooling radius, that since a cluster with $t_{\text {cool }}(0)>7.7 h_{71}^{-1 / 2}$ will, by definition, have $\dot{M}_{\text {classical }}=0$, it makes a poor parameter for comparison studies.

\subsubsection{Cuspiness (I): $\alpha$}

The histogram for cuspiness (defined in Eq. (1)) appears to have a Gaussian distribution around a mean at $\sim 1.2$. There seems to also be a broad distribution at $\sim 0.4$ and a sharp peak at $\sim 0.9$. The single Gaussian hypothesis is rejected by the KMM algorithm at $\sim 99 \%$ confidence. The LRTS suggests that the bimodal distribution is not as significant as for other parameters (e.g. 
$\left.\dot{M}_{\text {classical }} / M_{500}\right)$. Using a trimodal distribution is a significant improvement, but produces an unphysical distribution. Basically four of the CC clusters centered around $\sim 1.2$ are separated out of the CC subgroup (i.e. there are CC clusters on both sides of the four clusters, as opposed to them being between SCC and NCC clusters). For the bimodal distribution, the partition of the subgroups is $\sim 0.7$ which divides the sample into $35 \mathrm{CC}$ clusters and 29 NCC clusters. The centers of the subgroups are 1.07 and 0.412 for the CC and NCC clusters respectively. Vikhlinin et al. (2007) suggested a break of $\alpha=0.5$ for CC versus NCC clusters and $\alpha>0.7$ for SCC clusters. Using these cuts there are $45 \mathrm{CC}$ clusters (10 of which are weak CC clusters) and 19 NCC clusters.

We note here that for many of the clusters that we have in common with Vikhlinin et al. (2007), we generally find larger values of $\alpha$ than they do (based in Fig. 2 in Vikhlinin et al. 2007). There are several possible explanations for this discrepancy. The most obvious is the different way in which we calculated $r_{500}$. We calculated $r_{500}$ from $T_{\text {vir }}$ using the formula of Evrard et al. (1996), which, as we noted earlier, may overestimate $r_{500}$. On the other hand Vikhlinin et al. (2007) estimate $r_{500}$ from their mass model. If our value of $r_{500}$ is larger than the value used by Vikhlinin et al. (2007), it makes sense that our values of $\alpha$ will be larger, since the profile should steepen around $0.04 r_{500}$ (see Fig. 1 in Vikhlinin et al. 2007). The second major difference is that our values of $\alpha$ are derived from surface brightness profile models, whereas Vikhlinin et al. (2007) use direct deprojection and derive $\alpha$ from density profile models. Finally there are minor points that can contribute to the discrepancies, such as the center used to create the profile (we both use the X-ray peak, but for some NCC clusters this is not well-defined), the energy band for the surface brightness profile and the techniques used to create the profiles. In the end, we argue that these differences lead to an intrinsic scatter in the values of $\alpha$ obtained which are dependent on the method used to determine it.

\subsubsection{Scaled core luminosity (J): $L_{x} /\left[M_{\text {gas }} k T_{\text {vir }}\right]$}

We define a scaled version of central luminosity $L_{X}$. Scaled $L_{X}$ is taken from the projected spectral fit to the $0-0.048 r_{500}$ region. This luminosity is then scaled by $k T_{\text {vir }}$ and the gas mass of the region, calculated from the gas density profile $\left(L_{\mathrm{X}} /\left[M_{\text {gas }} k T_{\mathrm{vir}}\right]\right)$. The histogram of the scaled $L_{\mathrm{X}}$, appears to be a single distribution with a peak at $\sim 0.6 \times 10^{30} h_{71}^{1 / 2} \mathrm{erg} \mathrm{s}^{-1} \mathrm{keV}^{-1} M_{\odot}^{-1}$. In fact, the $\mathrm{KMM}$ algorithm does not reject the single Gaussian hypothesis ( $<40 \%$ confidence). If split into a bimodal distribution the subgroups are partitioned at $0.4 \times 10^{30} h_{71}^{1 / 2} \mathrm{erg} \mathrm{s}^{-1} \mathrm{keV}^{-1} M_{\odot}^{-1}$ which divides the sample into $49 \mathrm{CC}$ clusters and 15 NCC clusters. The centers of the subgroups are $0.72 \times 10^{30} h_{71}^{1 / 2} \mathrm{erg} \mathrm{s}^{-1} \mathrm{keV}^{-1} M_{\odot}^{-1}$ and $0.33 \times 10^{30} h_{71}^{1 / 2} \mathrm{erg} \mathrm{s}^{-1} \mathrm{keV}^{-1} M_{\odot}^{-1}$ for the CC and NCC clusters respectively.

\subsubsection{Central temperature drop (K): $T_{0} / T_{\text {vir }}$}

The central temperature drop $\left(T_{0} / T_{\text {vir }}\right)$ is often used as an identifier for CC clusters (e.g. Sanderson et al. 2006a; Burns et al. 2008). In the histogram there seems to be a large peak centered at $\sim 1$ and a broad range of temperature drops that peaks around $\sim 0.4$. The KMM algorithm rejects the single Gaussian hypothesis at $>99 \%$ confidence and the LRTS suggests that $T_{0} / T_{\text {vir }}$ has one of the most significant bimodal distributions. The partition between CC and NCC clusters is $\sim 0.7$, which divides the sample into $24 \mathrm{CC}$ clusters and 40 NCC clusters. The centers of the subgroups are 0.44 and 0.97 for the CC and NCC clusters respectively. For their sample of simulated clusters, Burns et al. (2008) made a cut at $T_{0} / T_{\text {vir }}=0.8$, which also seems to be a plausible cut for our sample.

\subsubsection{Slope of the inner temperature profile (L)}

The histogram for the slopes of the inner temperature profiles does not seem to show any bimodality, suggesting that there is no universal central temperature profile. The fact that $T_{0} / T_{\text {vir }}$ shows some evidence of bimodality hints that the lowest temperature of the gas in the center of the clusters never cools much below $\sim 0.4 T_{\text {vir }}$. However since the slopes show no evidence of bimodality, the gradient of this temperature drop from $T_{\text {vir }}$ to $0.4 T_{\text {vir }}$ is not universal. This would imply that the size of the region where the temperature drops below $T_{\text {vir }}$ is not universal (see Sect. 4.2 for a more detailed discussion). Usually, NCC clusters are thought to have no temperature drop, but the distribution of $\log \left(T_{0} / T_{\text {vir }}\right)$ is continuous from 0 to $\sim-0.2$, making it difficult to define a clean break. We also do not find any peak or distribution around the universal value of -0.4 found by Sanderson et al. (2006a). The KMM algorithm rejects the single Gaussian hypothesis at $>99 \%$ confidence, but the LRTS suggests the bimodality is not as significant as in other parameters (e.g. $T_{0} / T_{\text {vir }}$ ). The CC subgroup has a very large variance, suggesting a broad distribution in the inner $T$-profile slopes for CC clusters. In fact because of the large variance in the CC subgroup, the KMM algorithm identifies the three clusters with the steepest centrally increasing temperature profiles as belonging to the CC subgroup (see Fig. 4L). Since this is unphysical, we take the partition value to be -0.23 , which divides the sample into $15 \mathrm{CC}$ clusters and $49 \mathrm{NCC}$ clusters. The centers of the subgroups are -0.24 and -0.08 for the CC and NCC clusters respectively.

\subsubsection{Ratio of central temperatures in the soft band to the hard band (M): [ $\left.T_{0}(0.5-2.0 \mathrm{keV})\right] /\left[T_{0}(2.0-7.0 \mathrm{keV})\right]$}

We also considered fitting the same region to different energy bands in order to identify cool cores. The idea is that if there are many temperatures in the central region the soft band will be more sensitive to the cool gas, whereas the harder band will be more sensitive to the hotter gas. We took our central annulus from the $T$-profile and fit it in the $0.5-2.0 \mathrm{keV}$ band and then in the $2.0-7.0 \mathrm{keV}$ band and found the ratio. The distribution of values does not look bimodal at all and appears to be a Gaussian centered on $\sim 0.7$. However, the KMM algorithm rejects the single Gaussian hypothesis at $>99 \%$ confidence. On the other hand, the LRTS suggests the bimodality is not as significant as in other parameters (e.g. $T_{0} / T_{\text {vir }}$ ). As with $\dot{M}_{\text {spec }} / M_{500}$ and the slopes of the central temperature profiles, the large variance of one of the subgroups (the NCC subgroup in this case) causes some unphysical assignments (see Fig. 4M). The four cases with the smallest ratio of $\left[T_{0}(0.5-2.0 \mathrm{keV})\right] /\left[T_{0}(2.0-7.0 \mathrm{keV})\right]$ are assigned to the NCC subgroup. Using only the clusters with large values of $\left[T_{0}(0.5-2.0 \mathrm{keV})\right] /\left[T_{0}(2.0-7.0 \mathrm{keV})\right]$ that the KMM algorithm assigns to the NCC subgroup, the CC/NCC clusters are partitioned at $\sim 0.86$. This divides the sample into $50 \mathrm{CC}$ clusters and 14 NCC clusters. The centers of the subgroups are 0.72 and 0.87 for the CC and NCC clusters respectively. 


\subsubsection{Scaled gas mass within $0.048 r_{500}(\mathrm{~N}): M_{\text {gas }} / M_{500}$}

The high density in the centers of CC clusters suggests that there should be relatively more gas in the center compared with NCC clusters. The distribution, however, does not look particularly bimodal, suggesting that the size of the core does not scale with mass. There seems to be a peak at $\sim 0.4 \times 10^{-3}$, with several smaller peaks on either side. The KMM algorithm does not reject the single Gaussian hypothesis ( $<76 \%$ confidence). If split into two distributions they are partitioned at $\sim 0.45 h^{-3 / 2} \times 10^{-3}$, which divides the sample into $38 \mathrm{CC}$ clusters and $26 \mathrm{NCC}$ clusters. The centers of the subgroups are $0.87 h^{-3 / 2} \times 10^{-3}$ and $0.28 h^{-3 / 2} \times$ $10^{-3}$ for the CC and NCC clusters respectively.

\subsubsection{Scaled modified spectral mass deposition rate $(\mathrm{O})$ : $\dot{M}_{\text {spec } 2} / M_{500}$}

The histogram for $\dot{M}_{\text {spec } 2} / M_{500}$ is similar to the other $\dot{M}$ histograms although the upper peak is broader and not as pronounced. Interestingly, the scale of $\dot{M}_{\text {spec } 2} / M_{500}$ is similar to that of $\dot{M}_{\text {classical }} / M_{500}$ about an order of magnitude above $\dot{M}_{\text {spec }} / M_{500}$ (see also Figs. 10 and 15). As with the other $\dot{M}$ histograms, the clusters with $\dot{M}_{\text {spec2 }}=0$, were assigned to $\log \left[\dot{M}_{\text {spec } 2} /\left(M_{500} / 10^{-14} h_{71} \mathrm{yr}^{-1}\right)\right]=-5$ when applying the KMM algorithm to the log of data. The KMM algorithm only marginally rejects the single Gaussian hypothesis ( $\sim 90 \%$ confidence). The two subgroups are partitioned at $1.5 \times 10^{-14} h_{71} \mathrm{yr}^{-1}$, which divides the sample into $44 \mathrm{CC}$ clusters and 20 NCC clusters. The centers of the subgroups are $11.7 \times 10^{-14} h_{71} \mathrm{yr}^{-1}$ and $0.39 \times 10^{-14} h_{71} \mathrm{yr}^{-1}$ for the CC and NCC clusters respectively.

\subsubsection{Central cooling time $(P)$}

The histogram for CCT looks similar to the histogram of $K_{0}$, which is not surprising since they are calculated from similar quantities: $T_{\text {ctr }}{ }^{14}$ and $n_{0}$. Like $K_{0}$, the histogram of CCT has two peaks with a smaller peak between the two. The KMM algorithm rejects the single Gaussian hypothesis at $>99 \%$ confidence and the LRTS suggests the bimodality of CCT is the second most significant (after $\dot{M}_{\text {classical }} / M_{500}$ ) among the 16 parameters. As with $K_{0}$, adding a third subgroup increases the LRTS. For a bimodal distribution the partition is $\sim 5 h_{71}^{-1 / 2} \mathrm{Gyr}$, which divides the sample into $42 \mathrm{CC}$ clusters and $22 \mathrm{NCC}$ clusters. For the tri-modal distribution the CC/NCC partition remains at $\sim 5 h_{71}^{-1 / 2}$ Gyr and the SCC/WCC partition is $\sim 1 h_{71}^{-1 / 2}$ Gyr. There are four clusters with CCT between $5 h_{71}^{-1 / 2}$ Gyr and $7.7 h_{71}^{-1 / 2} \mathrm{Gyr}$, so that the difference between a partition at $5 h_{71}^{-1 / 2} \mathrm{Gyr}$ and $7.7 h_{71}^{-1 / 2} \mathrm{Gyr}$ is a matter of low number statistics. Since $7.7 h_{71}^{-1 / 2}$ Gyr corresponds to a look back time for $z \sim 1$, about the time most clusters would have time to relax and form a cool core, we decided to take the partition as $7.7 h_{71}^{-1 / 2}$ Gyr rather than $5 h_{71}^{-1 / 2}$ Gyr. Moreover we used $7.7 h_{71}^{-1 / 2}$ Gyr to determine $\dot{M}_{\text {classical }}$ and cooling radius. Using 1 and $7.7 h_{71}^{-1 / 2} \mathrm{Gyr}$ as cuts we divide the sample into 28 SCC clusters, 18 WCC clusters and 18 NCC clusters. The centers of the subgroups are $0.45 h_{71}^{-1 / 2} \mathrm{Gyr}, 1.91 h_{71}^{-1 / 2} \mathrm{Gyr}$ and $11.2 h_{71}^{-1 / 2}$ Gyr for the CC, WCC and NCC clusters respectively.

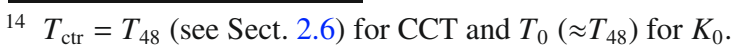

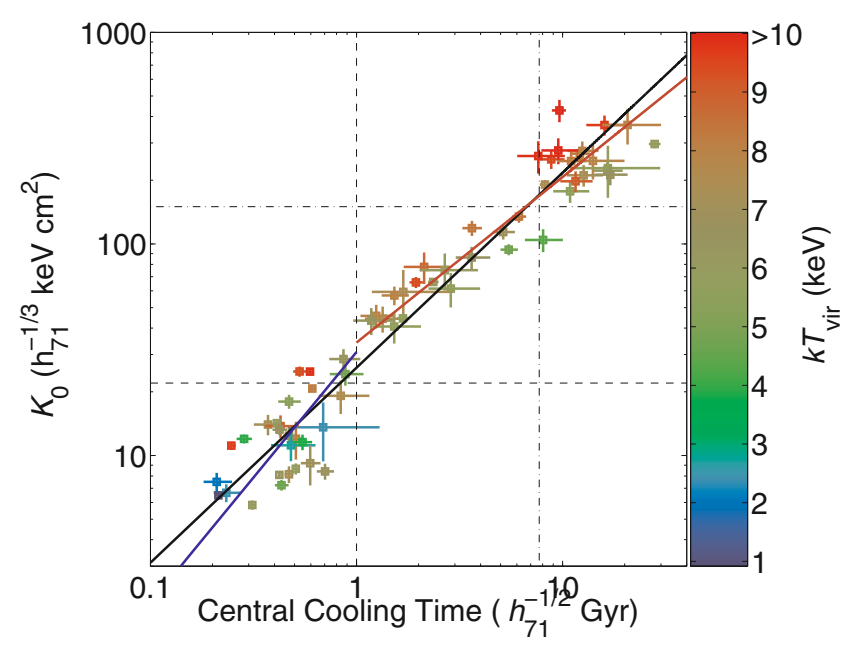

Fig. 5. This plot shows central entropy $\left(K_{0}\right)$ versus CCT. Since both quantities are derived from $T_{\text {ctr }}\left(T_{48} \approx T_{0}\right)$ and $n_{0}$, they are, not surprisingly, tightly correlated. The dashed lines show the division between $\mathrm{CC}$ and WCC clusters. The dash-dot lines show the division between WCC and NCC clusters. The black line shows the best fit for all clusters, the blue line shows the best-fit line for the SCC clusters and the red line shows the best fit to the combined NCC and WCCs. The data points are color coded by virial temperature (see scale at right).

\subsection{The defining parameter}

The likelihood ratio test identifies $\dot{M}_{\text {classical }} / M_{500}$ as having the most significant bimodality, however we did not choose it as the best method to separate NCC and CC clusters. There are two reasons for rejecting it as the best method. (1) Clusters which have a CCT $>7.7 h_{71}^{-1 / 2}$ Gyr have $\dot{M}_{\text {classical }}=0$, making it difficult to compare $\dot{M}_{\text {classical }} / M_{500}$ to other parameters. (2) The errors in $\dot{M}_{\text {classical }} / M_{500}$ are quite large (see Fig. 6G). In fact the average uncertainty in $\dot{M}_{\text {classical }} / M_{500}$ is $\sim 60 \%$ versus $15 \%$ for CCT, the parameter with the next most significant bimodality. This uncertainty is not accounted for in either the histogram or the KMM algorithm and therefore the significance of the bimodality and the cluster assignments are also quite uncertain.

The parameters with the next highest significance of bimodality are CCT and $K_{0}$. As noted earlier, $K_{0}$ and CCT are both calculated from $n_{0}$ and $T_{\text {ctr }}$ (in general $T_{48} \approx T_{0}$ ) and so it is not surprising that they have similar distributions. Figure 5 shows the tight correlation between $K_{0}$ and CCT. The dashed lines show the cut between CC and WCC clusters for each parameter and the dot-dashed lines show the cut between WCC and NCC clusters. There are four clusters which are classified as WCC clusters when using $K_{0}$ and are classified as SCC clusters when using CCT. It is interesting to note that these four clusters have the lowest value of $K_{0}$ for any of the WCC clusters (as classified by $K_{0}$ ) and there is also a clear break (at $\sim 30 h_{71}^{-1 / 3} \mathrm{keV} \mathrm{cm}^{2}$ ) between these clusters and the other WCC clusters (see Fig. 5). As noted earlier this break has also been reported by Hudson \& Reiprich (2007), Reiprich \& Hudson (2007), Voit et al. (2008), and Cavagnolo et al. (2009). Additionally one borderline WCC cluster is classified as an NCC cluster when using $K_{0}$ and one borderline NCC cluster is assigned to the WCC subpopulation when classified with $K_{0}$. Other than these six borderline cases, all the clusters are assigned to the same subpopluations whether CCT or $K_{0}$ is used to classify them.

Based in Fig. 5, there is not much difference between sorting the sample using $K_{0}$ or CCT. Assuming bremsstrahlung emission 
Table 4. Summary of the KMM algorithm results for the 16 parameters.

\begin{tabular}{|c|c|c|c|c|c|}
\hline Parameter & $\begin{array}{l}\text { Break } \\
\text { Value }^{\dagger} \\
\text { (2) } \\
\end{array}$ & $\begin{array}{c}\mu_{1} \\
\mathrm{CC} \\
\text { Centroid }^{\ddagger} \\
(3) \\
\end{array}$ & $\begin{array}{c}\mu_{2} \\
\text { NCC } \\
\text { Centroid } \\
(4) \\
\end{array}$ & $\begin{array}{l}\text { \# of CC } \\
\text { clusters }^{\alpha}\end{array}$ & $\begin{array}{c}\text { \# of NCC } \\
\text { clusters }\end{array}$ \\
\hline$\Sigma_{0}\left(10^{-6}\right.$ photons $\left.\mathrm{cm}^{-2} \mathrm{~s}^{-1} \operatorname{arcsec}^{-2}\right)$ & 0.80 & 3.88 & 0.32 & 37 & 27 \\
\hline$r_{\mathrm{c}} / r_{500}\left(10^{-2}\right)$ & 1.3 & 0.35 & 4.31 & 30 & 34 \\
\hline$n_{0}\left(10^{-2} h_{71}^{1 / 2} \mathrm{~cm}^{-3}\right)$ & 1.5 & 4.02 & 0.46 & 37 & 27 \\
\hline$K_{0}\left(h_{71}^{-1 / 3} \mathrm{keV} \mathrm{cm}^{2}\right)$ & $150(22)$ & $11(59)$ & 257 & $46(22)$ & 18 \\
\hline$K_{\text {BIAS }}\left(h_{71}^{-1 / 3} \mathrm{keV} \mathrm{cm}^{2}\right)$ & 40 & 11 & 131 & 29 & 35 \\
\hline Cooling Radius $\left(\% r_{500}\right)$ & 4.3 & 8.1 & 1.2 & 37 & 27 \\
\hline$\dot{M}_{\text {spec }} / M_{500}\left(h_{71} 10^{-14} \mathrm{yr}^{-1}\right)$ & 0.23 & 1.3 & 0.045 & 43 & 21 \\
\hline$\dot{M}_{\text {classical }} / M_{500}\left(h_{71}^{-1} 10^{-14} \mathrm{yr}^{-1}\right)$ & 0.5 & 20.5 & 0.0106 & 43 & 21 \\
\hline$\alpha$ & $0.7^{\beta}$ & 1.07 & 0.41 & 35 & 29 \\
\hline$L_{\mathrm{X}} /\left[M_{\text {gas }} k T_{\text {vir }}\right]\left(10^{30} h_{71}^{1 / 2} \mathrm{erg} \mathrm{s}^{-1} \mathrm{keV}^{-1} M_{\odot}^{-1}\right)$ & 0.4 & 0.72 & 0.33 & 49 & 15 \\
\hline$T_{0} / T_{\mathrm{vir}}$ & 0.7 & 0.97 & 0.44 & 24 & 40 \\
\hline Slope of Central Temperature Profile & -0.23 & -0.24 & -0.08 & 15 & 49 \\
\hline$\left[T_{0}(0.5-2.0 \mathrm{keV})\right] /\left[T_{0}(2.0-7.0 \mathrm{keV})\right]$ & 0.86 & 0.72 & 0.87 & 50 & 14 \\
\hline$M_{\text {gas }} / M_{500}\left(h_{71}^{-3 / 2} 10^{-3}\right)$ & 0.45 & 0.87 & 0.28 & 38 & 26 \\
\hline$\dot{M}_{\mathrm{spec} 2} / M_{500}\left(h_{71} 10^{-14} \mathrm{yr}^{-1}\right)$ & 1.5 & 11.7 & 0.39 & 44 & 20 \\
\hline Central Cooling Time $\left(h_{71}^{-1 / 2} \mathrm{Gyr}\right)$ & $7.7(1)$ & $0.45(1.91)$ & 11.2 & $46(18)$ & 18 \\
\hline
\end{tabular}

Notes. Columns: (1) the parameter, (2) the value of the parameter that separates CC and NCC clusters, (3) the KMM determined centroid value for the CC clusters, (4) the KMM determined centroid value for the NCC clusters, (5) the number of CC clusters determined by the KMM algorithm and (6) the number of NCC clusters determined by the KMM algorithm.

$\dagger$ The number in parenthesis, if any, is the cut between SCC and WCC (or transition) clusters.

$\$$ The number in parenthesis, if any, is the centroid value for the WCC clusters. In these cases the other number is the centroid value of the SCC clusters.

${ }^{\alpha}$ This number includes SCC and WCC clusters where appropriate. The number in parenthesis, if any, is the number of WCC (or transition) clusters.

${ }^{\beta}$ Using the cuts suggested by Vikhlinin et al. (2007) of $\alpha=1$ (WCC and SCC) and $\alpha=0.5$ (CC and NCC), gives 35 SCC clusters, 10 WCC clusters and 19 NCC clusters.

(i.e. $\Lambda(T) \propto T^{1 / 2}$ ) and $n_{i} \approx n_{\mathrm{e}} \equiv n$, the dependency of the cooling time on the temperature and density is given by $t_{\text {cool }} \propto T^{1 / 2} n^{-1}$. Therefore CCT is more dependent on $n$ than on $T$, while $K_{0}$ is more affected by $T$. Since the determination of $T$ is more resolution dependent (i.e. it requires $\sim 100 \times$ more photons to make a spectrum than one bin in a surface brightness profile), we argue that $t_{\text {cool }}$ is a better parameter to use. Furthermore it is also a more traditional metric and short cooling times are the physical basis of the cooling flow problem.

\subsubsection{CC fractions in redshift and temperature}

We further investigated the subpopulations in the CCT by applying the Wilcoxon rank-sum test (Wilcoxon 1945; Mann \& Whitney 1947) to the redshift and $T_{\text {vir }}$ distributions of the CC (as well as the SCC and WCC subsamples) and NCC clusters. The Wilcoxon rank-sum test is a non-parametric test that determines whether the values of a parameter of two subpopulations derive from the same parent distribution. For instance, it can be used to determine if the redshifts of the CC and NCC clusters come from the same parent distribution of redshifts. The results are presented in Table 5. We find that within $1 \sigma$, the CC and NCC redshifts derive from the same redshift population. That is, there is no evolution of the $\mathrm{CC} / \mathrm{NCC}$ fraction within our redshift range. Likewise, when comparing the redshift distributions of WCC and NCC clusters and SCC and WCC clusters, the redshifts, within $1 \sigma$, derive from the same population. However, for SCC and NCC clusters there is a marginally significant difference $(\sim 1.1 \sigma)$ in the redshift distributions, with NCC clusters having generally higher redshifts. Naïvely, this seems to indicate that the fraction of SCC to NCC clusters is higher at lower redshift. However, when examining the $T_{\text {vir }}$ populations, it is clear that CC (and SCC) clusters come from a lower temperature population than NCC clusters, with $\sim 2 \sigma$ significance ( $\sim 2.4 \sigma$ for SCC clusters). There is even a significant difference $(\sim 1.6 \sigma)$ between $T_{\text {vir }}$ for SCC and WCC clusters. Only WCC and NCC clusters seem to derive from the same population of $T_{\text {vir }}$. Since our sample is flux-limited, low temperature clusters will only appear at low redshifts. Since SCC clusters come from a significantly cooler population than NCC clusters, it makes sense that they would also have a lower redshift distribution in a flux-limited sample. Therefore we conclude that within our redshift range, there is no evolution in the $\mathrm{CC}$ fraction. We also find low temperature clusters/groups are more likely to be CC clusters than high temperature clusters, consistent with the results of Burns et al. (2008) and Chen et al. (2007).

\subsubsection{Bias on CC fractions due to flux-limited nature of the sample}

Flux-limited samples suffer from the well-known Malmquist bias, namely that brighter objects have a higher detection rate than fainter objects. In this section, we address how this bias may affect the observed fractions of SCC, WCC and NCC clusters.

Strong cool-core clusters, owing to their high central densities have enhanced central X-ray emission. This may result in a higher chance of their detection and serve as an explanation for observing a higher fraction of SCC clusters in the HIFLUGCS and other flux- or luminosity-limited samples. Since we have a complete sample, this calculation can be done. We simulated samples of clusters which follow the X-ray temperature function given by $\mathrm{d} N / \mathrm{d} V \sim T^{-3.2}$ (Ikebe et al. 2002), in the temperature 
Table 5. Results to the Wilcoxon rank-sum test applied to redshift and $T_{\text {vir }}$ for the CC, SCC, WCC and NCC subpopulations.

\begin{tabular}{|c|c|c|c|}
\hline $\begin{array}{l}\text { Subsamples } \\
\text { being } \\
\text { compared } \\
\text { (1) }\end{array}$ & $\begin{array}{l}\text { Parameter } \\
\text { being } \\
\text { used } \\
(2)\end{array}$ & $\begin{array}{c}\text { Significance } \\
(\sigma) \\
(3)\end{array}$ & $\begin{array}{l}\text { Which subsample } \\
\text { has a larger avg. } \\
\text { param. value? } \\
\text { (4) }\end{array}$ \\
\hline CC-NCC & redshift & 0.82 & $\mathrm{NCC}$ \\
\hline SCC-NCC & redshift & 1.12 & NCC \\
\hline SCC-WCC & redshift & 0.96 & WCC \\
\hline WCC-NCC & redshift & 0.16 & NCC \\
\hline CC-NCC & $T_{\text {vir }}$ & 1.98 & $\mathrm{NCC}$ \\
\hline $\mathrm{SCC}-\mathrm{NCC}$ & $T_{\mathrm{vir}}$ & 2.41 & $\mathrm{NCC}$ \\
\hline SCC-WCC & $T_{\mathrm{vir}}$ & 1.62 & WCC \\
\hline WCC-NCC & $T_{\mathrm{vir}}$ & 0.81 & NCC \\
\hline
\end{tabular}

Notes. Columns: (1) subsamples being compared, (2) the parameter (redshift or $T_{\text {vir }}$ ) being used for the comparison, (3) the significance that the two distributions are not consistent with the null hypothesis (they are from the same parent distribution), (4) the subpopulation that comes from the larger redshift or higher temperature distribution.

Table 6. Results of simulations done to investigate the impact of selection effects on the observed fractions of SCCs, WCCs, and NCCs.

\begin{tabular}{cccc}
\hline \hline Case & Category & $\begin{array}{c}\text { Input } \\
\text { fractions }\end{array}$ & $\begin{array}{c}\text { Output } \\
\text { fractions }\end{array}$ \\
\hline Same slope & SCC & 0.34 & $0.437 \pm 0.043$ \\
& WCC & 0.36 & $0.281 \pm 0.041$ \\
& NCC & 0.30 & $0.281 \pm 0.044$ \\
\hline Different slopes & SCC & 0.310 & $0.445 \pm 0.041$ \\
& WCC & 0.335 & $0.275 \pm 0.038$ \\
& NCC & 0.355 & $0.280 \pm 0.041$ \\
\hline
\end{tabular}

range $(0.001-15) \mathrm{keV}$ and redshift range $0.00-0.25$. From the above it is clear that SCC, WCC and NCC clusters come from the same parent redshift distribution within $1-\sigma$ standard deviation. Hence, we assigned to the clusters random redshifts conforming to the $N \propto D_{\mathrm{L}}^{3}$ law, where $D_{\mathrm{L}}$ is the luminosity distance. We calculated the luminosities using three different $L_{X}-T_{\text {vir }}$ relations as determined for each of the three categories, the SCC, WCC, and NCC clusters, individually (Mittal \& Reiprich, in preparation).

In order to estimate the effect of imposing a flux-limit to a mixed sample of SCCs, WCCs and NCCs on their resulting fractions, we applied the HIFLUGCS flux-limit, $f_{\mathrm{X}}(0.1-2.4) \mathrm{keV} \geq$ $2 \times 10^{-11} \mathrm{erg} \mathrm{s}^{-1} \mathrm{~cm}^{-2}$, to the simulated sample. We tried two different input sets. In the first (simplest) case, we assumed the SCCs, WCCs and NCCs to have the same $L_{X}-T_{\text {vir }}$ slope (3.33). The normalizations were fixed to those found from the fits to the data. In the second case, we fixed the slopes for SCCs, WCCs and NCCs to the fitted values (Mittal \& Reiprich, in preparation). We find that in both the cases the output fractions are indeed biased. In particular, the output SCC fraction is higher than the input value. Thus we conclude that in reality the SCCs, WCCs and NCCs may occur with similar fractions and due to the increased $\mathrm{X}$-ray luminosity in SCCs, their observed fraction is higher in the present sample.

\subsection{Cooling time compared to other parameters}

In order to study underlying physical relations and correlate simple observables with those requiring high quality data, we compare 14 parameters ${ }^{15}$ from Fig. 4 to the CCT. The relations are

\footnotetext{
15 We already compared $K_{0}$ to the CCT in Fig. 3.2, so it is not repeated here.
}

plotted in Fig. 6. The points are color coded by virial temperature with the color scale cropped at $10 \mathrm{keV}$. In order to quantitatively compare the parameters to CCT, we fit the relations to powerlaws ${ }^{16}$. Since there were errors on both the parameters and CCT, we used the bisector linear regression routine, BCES (Akritas \& Bershady 1996), to fit the data. Although some of the parameters (e.g. $K_{0}$ ) had correlated errors with CCT, for simplicity we assumed them to be independent. Additionally, since the relation between the CCT and the other parameters seemed to behave differently for the SCC clusters, we separately fit the SCC clusters and the non-SCC (WCC and NCC) clusters. The black line gives the best fit for all clusters, the blue line gives the best-fit line for the SCC clusters and the red gives best-fit line for the non-SCC clusters. Table 7 gives the fit values for all 14 parameters (along with $K_{0}$ ) for all three lines.

\subsection{1. $\Sigma_{0}$}

It has been suggested that the central surface brightness, $\Sigma_{0}$, is an indicator of CC strength (e.g. O'Hara et al. 2006). We find a surprising large amount of scatter in the plot of $\Sigma_{0}$ versus CCT, especially at short cooling times. Since $n_{0}$ is derived from $\Sigma_{0}$ and CCT is derived from $n_{0}$, one would naïvely expect a tighter correlation. Since $\Sigma \propto n^{2}$ any scatter in the $n_{0}$ versus CCT relation will be amplified in the $\Sigma_{0}$ versus CCT relation. Not surprisingly, the outliers at the low end are the lower temperature objects. Since CCT depends both on $n_{0}$ and $T_{0}$, cooler gas will have a shorter cooling time at the same density. Since cooler clusters are more likely to be CC clusters than hotter clusters (see Sect. 3.2), it is not surprising that there is more scatter for the SCC clusters than for WCC and NCC clusters. When using $\Sigma_{0}$ to identify CC clusters, the scatter will lead to misidentification; however, adding information about $T_{\text {vir }}$ may be used to help reduce the scatter.

If $\Sigma_{0}$ is used to separate the CC and NCC clusters, the WCC clusters are split into CC and NCC clusters, but not clearly on the basis of CCT. The WCC clusters with the longest CCTs are classified as NCC clusters, but the WCC clusters with intermediate CCTs $\left(\sim 4 h_{71}^{-1 / 2} \mathrm{Gyr}\right)$ and short CCTs $\left(\sim 1-2 h_{71}^{-1 / 2} \mathrm{Gyr}\right)$ are sometimes categorized as CC clusters and sometimes as NCC clusters. Figure 7 shows how the correlation becomes tighter if the central surface brightness is scaled by $k T_{\text {vir }}$. Cutting $\Sigma_{0} / k T_{\text {vir }}$ at $1.5 \times 10^{-7}$ photons cm $\mathrm{cm}^{-2} \mathrm{~s}^{-1} \mathrm{keV}^{-1}$ successfully separates the SCC and NCC clusters, with five misclassified WCC clusters, all but one (A2634) with relatively long CCTs $\left(>3 h_{71}^{-1 / 2}\right.$ Gyr).

\subsection{2. $r_{\mathrm{c}}\left(\% r_{500}\right)$}

We checked to see if there was any correlation between CCT and $r_{\mathrm{c}}$ as a percentage of $r_{500}$. We expected a correlation since $\mathrm{CC}$ clusters usually require a double $\beta$ model to fit their surface brightness profile, whereas NCC clusters do not. Therefore we expected $r_{\mathrm{c}} / r_{500}$ to be much smaller for CC clusters than NCC clusters. Surprisingly the correlation appears to be tighter than for $\Sigma_{0}$, although there is still a lot of scatter. The KMM determined cut would classify four SCC clusters as well as most of the WCC clusters as NCC clusters. If we put the cut at $0.03 r_{500}$ (just after the third peak in the histogram counting from the left), however, we would more successfully separate the CC clusters and NCC clusters. On the other hand, this parameter does a poor

\footnotetext{
${ }^{16}$ Some parameters are more nearly linear in the log of the cooling time, in which case the parameter was exponentiated before fitting.
} 

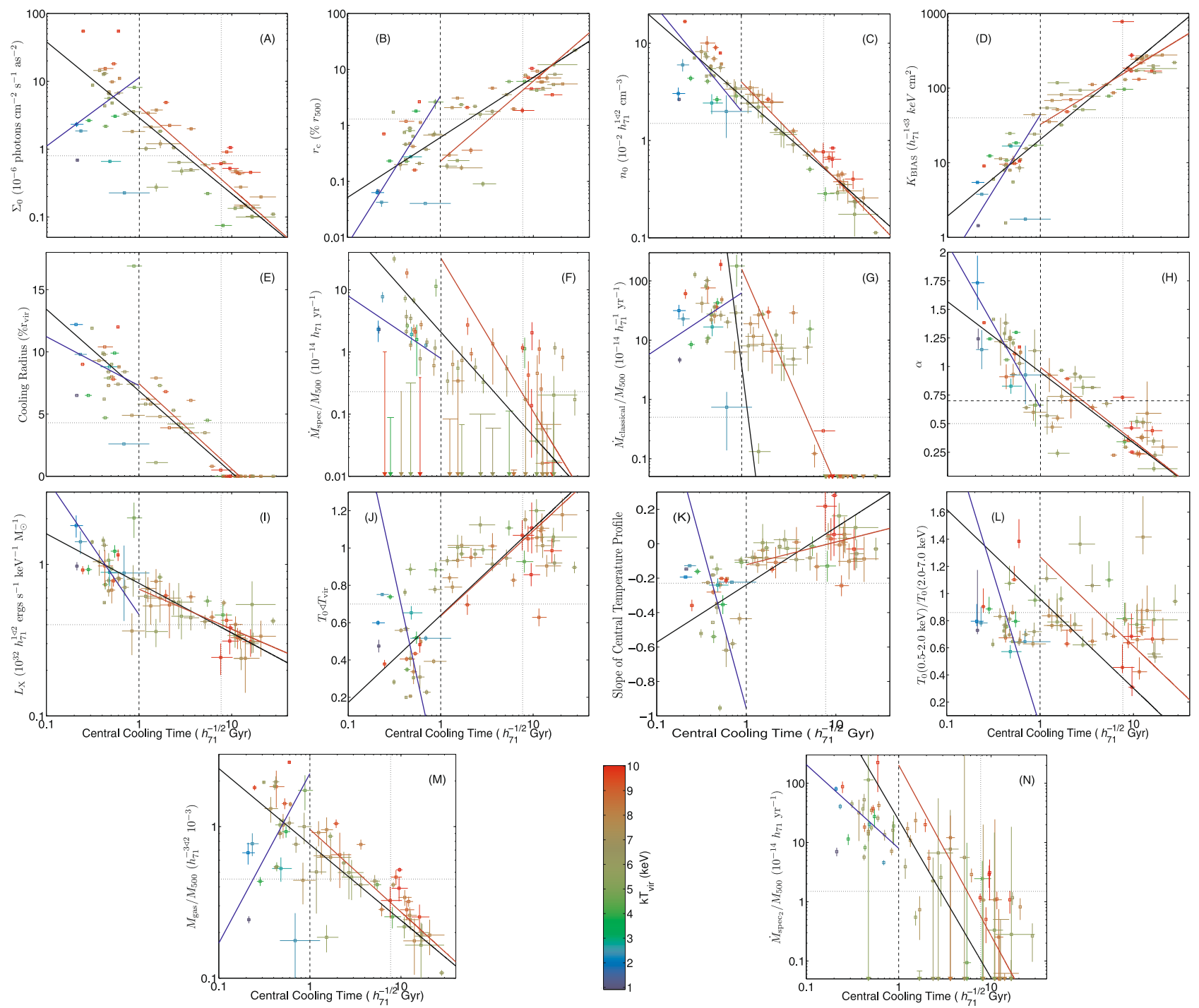

Fig. 6. A comparison of CCT with fourteen of the parameters from Fig. 4. Row-wise left to right and starting from the top row the plots are: A) central surface brightness $\left.\Sigma_{0} ; \mathbf{B}\right) \beta$ model core radius $r_{\mathrm{c}}$ as a percentage of $r_{500}$; C) central density $n_{0}$; D) biased central entropy $K_{\mathrm{BIAS}} ; \mathbf{E}$ ) cooling radius; F) spectral mass deposition rate $\dot{M}_{\text {spec }}$ scaled by $M_{500} ; \mathbf{G}$ ) classical mass deposition rate $M_{\text {classical }}$ scaled by $\left.M_{500} ; \mathbf{H}\right)$ cuspiness $\alpha ;$ I) scaled central, bolometric, X-ray luminosity $\left.L_{\mathrm{X}} /\left(M_{\mathrm{gas}} k T_{\mathrm{vir}}\right) ; \mathbf{J}\right)$ central temperature drop $\left.T_{0} / T_{\mathrm{vir}} \mathbf{K}\right)$ slope of the central temperature profile; $\left.\mathbf{L}\right)$ central soft band determined temperature divided by central hard band determined temperature $\left[T_{0}(0.5-2.0 \mathrm{keV})\right] /\left[T_{0}(2.0-7.0 \mathrm{keV})\right]$, (M) central gas mass $M_{\text {gas }}$ scaled by $M_{500}$ and (N) modified spectral mass deposition rate $\dot{M}_{\text {spec } 2}$ scaled by $M_{500}$. The clusters are color coded by virial temperature with the colorscale shown in the bottom center. The dotted lines represent the division between CC clusters and NCC clusters as determined by the KMM algorithm for that particular parameter. The dashed lines represent the division between SCC clusters and WCC clusters. The solid black line is the best fit to all the data, the blue line is the fit only to the SCC clusters (as determined by CCT) and the red line is the fit to the WCC and NCC clusters (as determined by CCT). As noted in the text, the Fornax cluster is often a strong outlier. See notes on individual parameters and Sect. C.10 for specifics.

job of separating SCC and WCC clusters. An interesting note is that the coolest clusters (groups) appear to have smaller values of $r_{\mathrm{c}} / r_{500}$ compared to hotter clusters.

\subsection{3. $n_{0}$}

There is a tight correlation between $n_{0}$ and CCT. This is not surprising since, as noted earlier, $n_{0}$ is the strongest factor in determining CCT. All the SCC clusters are CC clusters when determined with $n_{0}$ and, similarly, both methods agree on the NCC clusters as well. The WCC clusters are split at CCT $\lesssim$ $2 h_{71}^{-1 / 2}$ Gyr into nine $\mathrm{CC}$ and nine being NCC when determined by $n_{0}$. It is impossible to separate SCC and WCC clusters using $n_{0}$. This suggests that the difference between SCC and
WCC clusters with similar values of $n_{0}$ is that SCC clusters have a lower central temperature. This may be due to the central temperature drop seen in SCC clusters or simply that these SCC clusters are cooler than their WCC counterparts. Considering NCC and WCC clusters with similar values of $n_{0}$, the WCC clusters must be cooler than their NCC counterparts.

\subsection{4. $K_{\mathrm{BIAS}}$}

As with central entropy, $K_{\mathrm{BIAS}}$ seems to form a tight correlation with CCT. The KMM break separates the SCC clusters from the other clusters (with one exception). Perhaps the most interesting aspect of $K_{\mathrm{BIAS}}$ is that the slope seems to break between the CC and NCC clusters. Unlike many of the other parameters 


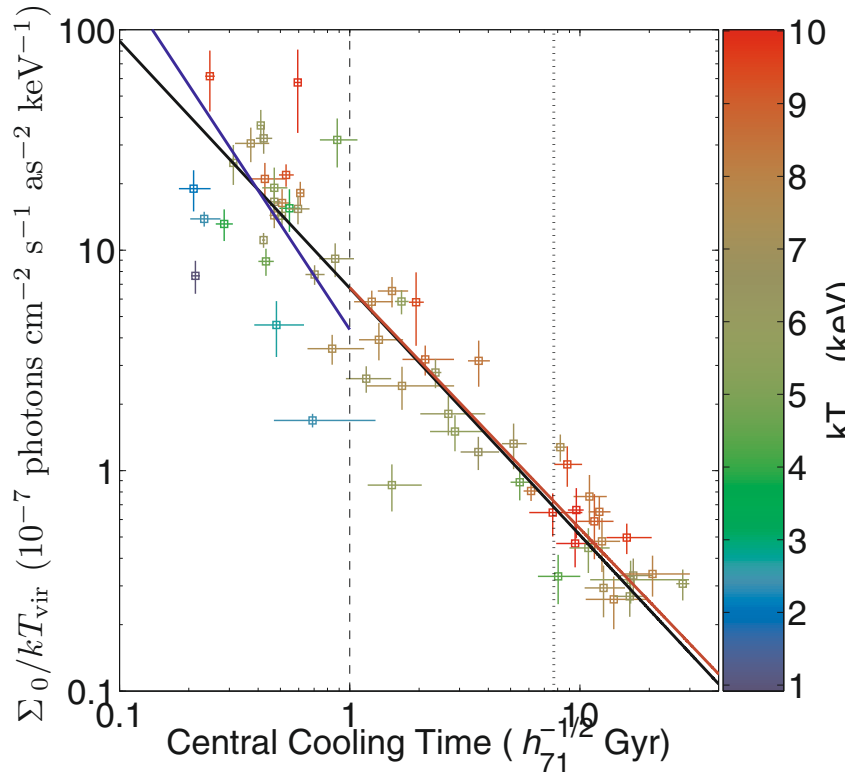

Fig. 7. The scaled central surface brightness $\Sigma_{0} / k T_{\text {vir }}$ versus CCT. The $\Sigma_{0}$ versus CCT correlation tightens when the $\Sigma_{0}$ is scaled by $k T_{\text {vir }}$.

this looks truly like a broken powerlaw, as fits to both the SCC and WCC/NCC clusters have similar values at the transition between them. This appears to be a good parameter for determining CC and NCC clusters, although ideally the redshift and observation length biases would need to be removed. The merging cluster A3266 is the outlier with CCT $\sim 7.7 h_{71}^{-1 / 2}$ and $K_{\text {BIAS }} \sim 900 h_{71}^{-1 / 3} \mathrm{keV} \mathrm{cm}^{2}$.

\subsubsection{Cooling radius $\left(\% r_{\mathrm{vir}}\right)$}

Since the cooling radius is defined at $t_{\text {cool }}\left(r_{\text {cool }}\right)=7.7 h_{71}^{-1 / 2} \mathrm{Gyr}$, the NCC clusters have no cooling radius by definition. These clusters were excluded when fitting the relation between cooling radius and CCT. There seems to be a trend of increasing cooling radius with decreasing cooling time for the WCC clusters. The SCC clusters, however, do not seem to have any trend between cooling time and size of the cooling region (as a fraction of the virial radius). Therefore we can conclude that the density gradients for SCC clusters vary greatly. The large outliers are: $\mathrm{S} 1101$ at the high end with a cooling radius of $>0.15 r_{500}$ and the Fornax cluster at the low end.

\subsubsection{Scaled spectral mass deposition rate}

This plot explicitly demonstrates the danger of using $\dot{M}_{\text {spec }}$ to divide the distribution. The error bars that are not visible in the histogram and not considered in the KMM-algorithm, show how much overlap there is within errors. There seems to be only a very weak trend with CCT, especially if the upper limits are included. The clusters with the highest values of $\dot{M}_{\text {spec }} / M_{500}$, however, are SCC clusters. As noted earlier and discussed in more detail later, several of the NCC clusters have non-negligible spectral mass deposition rates. This fact along with the spread in values for the SCC clusters makes it very difficult to use $\dot{M}_{\text {spec }}$ to identify CC and NCC clusters, at least with the spectral resolution of the Chandra ACIS.

\subsubsection{Scaled classical mass deposition rate}

As with cooling radius, $\dot{M}_{\text {classical }}$ is defined as zero for the NCC clusters (clusters with CCT $>7.7 h_{71}^{-1 / 2} \mathrm{Gyr}$ ), so these clusters were omitted when fitting the comparison of $\dot{M}_{\text {classical }} / M_{500}$ to CCT. There appears to be a weak trend of increasing $\dot{M}_{\text {classical }} / M_{500}$ with decreasing CCT, albeit with a great deal of scatter. We attribute this scatter to the differences in the gradients of the density profiles. That is, clusters with a very steep density gradient will have a very short CCT but not a large value of $\dot{M}_{\text {classical }}$, whereas clusters with relatively dense cores but flatter density gradients will have a longer CCT but a large $\dot{M}_{\text {classical }}$. Based on the KMM determined cut of $0.5 \times 10^{-14} h_{71}^{-1} \mathrm{yr}^{-1}$ for $\dot{M}_{\text {classical }} / M_{500}$, all the SCC clusters are classified as CC clusters and all but three of the WCC clusters are classified as CC clusters. Two of these WCC clusters are borderline cases.

There are six WCC clusters (IIIZw54, A1650, A1651, A2142, A2244 and A4038) that have $\dot{M}_{\text {classical }} / M_{500}>15 \times$ $10^{-14} h_{71}^{-1} \mathrm{yr}^{-1}$, more consistent with SCC clusters, although toward the lower end of the SCC clusters. There are five SCC clusters (A0262, MKW4, NGC 4636, A3526 and A1644) that have $\dot{M}_{\text {classical }} / M_{500}<15 \times 10^{-14} h_{71}^{-1} \mathrm{yr}^{-1}$, more consistent with WCC clusters. There are four (one SCC and three WCC clusters) outliers that have very low values of $\dot{M}_{\text {classical }} / M_{500}$ (i.e. $\left.<4 \times 10^{-14} h_{71}^{-1} \mathrm{yr}^{-1}\right)$. They are: NGC 1399, A3266, A3667 and A2634. It is interesting to note that all but two of the low $\dot{M}_{\text {classical }} / M_{500}$ SCC clusters are low temperature $(k T<$ $2.5 \mathrm{keV}$ ) systems, in fact they are four of the nine coolest systems. For the ten coolest systems, $\dot{M}_{\text {classical }} / M_{500}$ is less than $45 \times 10^{-14} h_{71}^{-1} \mathrm{yr}^{-1}$, which is below the average for the SCC clusters. The WCC outliers are all merging systems suggesting current cool-core survival, but with some disruption of the cool core.

\subsubsection{Cuspiness}

Cuspiness shows a lot of scatter when compared to CCT. It seems to do a good job in identifying NCC clusters; only three NCC clusters have $\alpha>0.5$. All the SCC clusters have $\alpha>0.5$, but not all of them have $\alpha>0.75$ as suggested by Vikhlinin et al. (2007). Using the KMM determined cut of $\alpha=0.7$, several of the SCC and all of the WCC would be classified as NCC clusters. Likewise, using the cuts of Vikhlinin et al. (2007), seven of the WCC clusters would be SCC clusters, three of the SCC clusters would be WCC clusters and four of the WCC clusters would be classified as NCC clusters. Looking at the fit to the data, there appears to be a shift in normalization and slope for the SCC clusters. That is, cuspiness increases with shorter CCTs. However, below CCT $\sim 1 h_{71}^{-1 / 2}$, the cuspiness drops suddenly (or at least there is a large range of values). Below a CCT of $\sim 1 h_{71}^{-1 / 2} \mathrm{Gyr}$, the cuspiness increases more rapidly as the CCTs get shorter.

\subsubsection{Scaled central luminosity}

Scaled central luminosity $\left(L_{\mathrm{X}} /\left[M_{\mathrm{gas}} k T_{\mathrm{vir}}\right]\right)$ shows a rather flat but clear relation with CCT, with large scatter and larger uncertainties. Using the KMM determined cut, however, only one SCC cluster is misclassified as an NCC cluster. Some of the WCC clusters are classified as CC clusters and some as NCC clusters when using the KMM determined cut in scaled central luminosity. If the cut is raised to $0.5 \times 10^{32} h_{71}^{1 / 2} \mathrm{erg} \mathrm{s}^{-1} \mathrm{keV}^{-1} M_{\odot}^{-1}$, still only one SCC cluster is misclassified, and the number of misclassified NCC clusters is 
reduced to two. Unlike, e.g. $n_{0}$, there seems to be no trend with the CCT of the WCC and whether it is classified as CC or NCC when using scaled central luminosity. As with many parameters the relation seems to get steeper for the SCC clusters.

\subsubsection{0. $T_{0} / T_{\text {vir }}$}

The most interesting feature in this plot is the break in the distribution for the SCC clusters. Although there is a lot of scatter, there is a clear drop in central temperature for SCC clusters. However, the value of the drop seems to be independent of CCT. There are a few NCC clusters with central temperature drops, most notably A2256. This may be due to a recent merger in which a core has not yet been completely destroyed (although a possible dense core has been disrupted).

Sanderson et al. (2006a) suggested a similar parameter for dividing CC and NCC clusters. Their parameter is roughly the inverse of $T_{0} / T_{\text {vir }}\left(T\right.$-ratio $\left.\equiv T_{\text {vir }} / T_{\text {core }}\right)$. The major difference being that $T_{\text {core }}$ is defined as the temperature within $0.1 r_{500}$, in general much larger than the region we used to measure $T_{0}$. They then define CC significance, which is the difference (in units of $\sigma$ ) between $T$-ratio and unity (i.e. $\frac{T \text {-ratio-1 }}{\sigma_{T \text {-ratio }}}$ ). They define a $\mathrm{CC}$ cluster as any cluster with $\mathrm{CC}$ significance $>3$ (i.e. a significant drop in temperature). Since they use a bright subsample of HIFLUGCS, all 20 of their clusters are in our sample. We find that all their CC clusters are SCC clusters in our sample. Their NCC clusters are either WCC or NCC clusters. Likewise, employing their method, using $T_{\mathrm{vir}} / T_{0}$, we find good agreement with their classification. On the other hand we argue the values of CC significance are very sensitive to the uncertainties in $T_{0}$ and $T_{\text {vir. }}$. That is if $T$-ratio $\lesssim 1$, then the longer the observing time the larger CC significance becomes and the more likely the cluster will be classified as a CC cluster. However, we concur with their result that in general SCC clusters have a central temperature drop and that WCC and NCC clusters have a shallow or no central temperature drop.

\subsubsection{Slope of the temperature profile}

Like $T_{0} / T_{\text {vir }}$, this plot shows a break in the distribution at the SCC clusters. It is clearer in this plot than in the $T_{0} / T_{\text {vir }}$ plot. This is most likely because the slope takes into account a systematic decrease in temperature as opposed to random temperature fluctuations. That is, for an NCC cluster with some cool gas that has not been completely disrupted, there will be less of a gradient than for a cluster that has a systematic decrease in temperature in a dense, well-defined core. For the SCC clusters there seem to be many slopes independent of CCT, suggesting no universal central temperature profile. This along with $T_{0} / T_{\text {vir }}$ demonstrates that studies that define CC clusters based on a temperature drop in the core are defining SCC clusters as CC clusters and WCC and NCC clusters as NCC clusters. The temperature drop and profiles are discussed in more detail in Sect. 4.2.

\subsubsection{2. $\left[T_{0}(0.5-2.0 \mathrm{keV})\right] /\left[T_{0}(2.0-7.0 \mathrm{keV})\right]$}

$\left[T_{0}(0.5-2.0 \mathrm{keV})\right] /\left[T_{0}(2.0-7.0 \mathrm{keV})\right]$ appears to be a very poor parameter for identifying CC clusters. As with $\dot{M}_{\text {spec }}$, it is possible that multiple temperatures along the line of sight (e.g. from a merging system) can produce results that make an NCC cluster appear to be a CC cluster. In fact the best fit (albeit a very poor fit) shows a positive trend rather than the expected negative

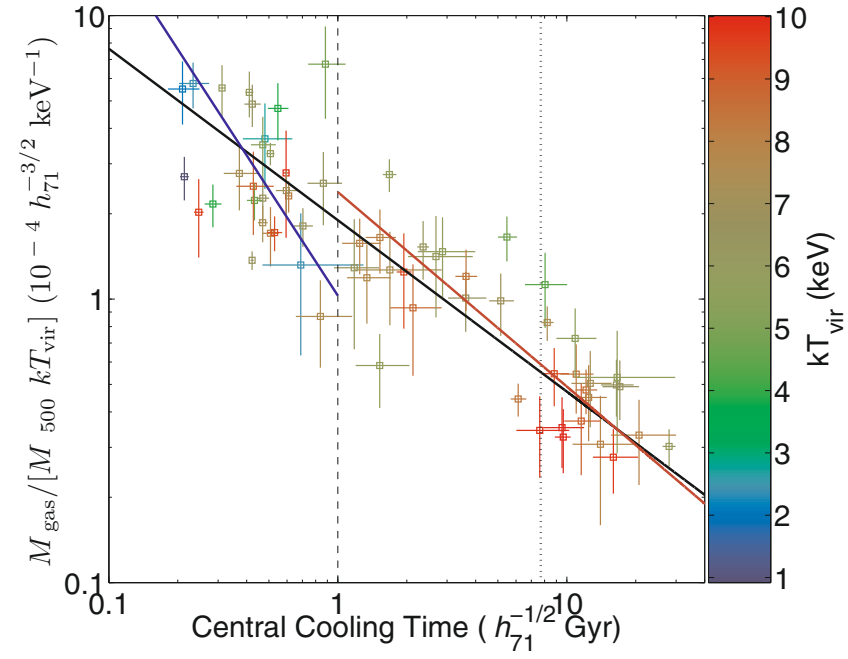

Fig. 8. This figure shows that that $M_{\mathrm{gas}} / M_{500}$ versus CCT correlation tightens for SCC clusters if scaled by $k T_{\text {vir }}$ This suggests that cool clusters cause the scatter in this relationship. Unfortunately, but not surprisingly, the tight correlation between $M_{\mathrm{gas}} / M_{500}$ and CCT for WCC and NCC clusters becomes worse.

trend. Additionally, an NCC cluster (A1656) shows the smallest fractional value.

\subsubsection{3. $M_{\text {gas }} / M_{500}$}

$M_{\text {gas }} / M_{500}$ shows a very promising trend for the WCC and NCC clusters; however, at CCT $\lesssim 1 h_{71}^{-1 / 2}$ Gyr there appears to be no relation. In fact, the best fit to the SCC clusters predicts a decrease in $M_{\text {gas }} / M_{500}$ with decreasing CCT. The outliers appear to be cool clusters and groups, which are less dense in the core (short cooling time comes partially from low temperature) and possibly have steeper profiles (leading to less gas mass within $0.048 r_{500}$ than for rich clusters). Figure 8 shows how dividing by $k T_{\text {vir }}$ lessens the scatter for the SCC clusters, explicitly showing that the large scatter is caused by cool clusters. Of course, since the correlation is already tight for NCC and WCC clusters, dividing by $k T_{\text {vir }}$ results in more scatter for those clusters.

\subsubsection{Scaled modified spectral mass deposition rate}

As with $\dot{M}_{\text {spec }} / M_{500}$, the large uncertainty in $\dot{M}_{\text {spec2 }} / M_{500}$ shows the limitations of the histogram. However, $\dot{M}_{\text {spec } 2} / M_{500}$ does show a trend with cooling time, albeit with a large scatter. The fact that it shows a trend is interesting since CCT and $\dot{M}_{\text {spec2 }}$ are independently determined. See Sect. 4.1 for a further discussion on $\dot{M}_{\text {spec2 }}$.

\subsection{What is the best diagnostic of CC clusters?}

One large problem in studying the evolution of CC cluster fraction is that resolving the cores of clusters is difficult for distant clusters. In Sect. 3.3 we compared various parameters to CCT. Not surprisingly $n_{0}$ and $K_{0}$ provide the tightest correlation to CCT. In distant clusters $n_{0}$ is slightly easier to determine than CCT and $K_{0}$ since it requires no spectral information from the central region. On the other hand it is still dependent on one being able to resolve the very center of the cluster, so it is not the ideal parameter to determine whether a distant cluster is a $\mathrm{CC}$ or NCC cluster. 
Table 7. The best-fit parameters for the 15 investigated CCT versus parameter. The parameters were fit to $\mathrm{CCT}=\mathrm{CCT}_{0}\left(\mathrm{parameter}^{\Gamma}\right.$.

\begin{tabular}{|c|c|c|c|c|c|c|}
\hline Parameter & $\begin{array}{c}\Gamma \\
\text { All }\end{array}$ & $\begin{array}{c}\mathrm{CCT}_{0} \\
\text { All } \\
h_{71}^{-1 / 2} \mathrm{Gyr} \\
\text { (3) }\end{array}$ & $\begin{array}{c}\Gamma \\
\text { SCC }\end{array}$ & $\begin{array}{c}\mathrm{CCT}_{0} \\
\mathrm{SCC} \\
h_{71}^{-1 / 2} \mathrm{Gyr} \\
(5)\end{array}$ & $\begin{array}{c}\Gamma \\
\mathrm{WCC}+\mathrm{NCC} \\
(6)\end{array}$ & $\begin{array}{c}\mathrm{CCT}_{0} \\
\mathrm{WCC}+\mathrm{NCC} \\
h_{71}^{-1 / 2} \mathrm{Gyr} \\
(7)\end{array}$ \\
\hline$K_{0} /\left(100 h_{71}^{-1 / 3} \mathrm{keV} \mathrm{cm}^{2}\right)$ & $1.085 \pm 0.036$ & $4.3 \pm 0.6$ & $0.843 \pm 0.109$ & $2.7 \pm 0.8$ & $1.278 \pm 0.073$ & $3.9 \pm 1.3$ \\
\hline$\Sigma_{0} /\left(10^{-6}\right.$ photons $\left.\mathrm{cm}^{-2} \mathrm{~s}^{-1} \operatorname{arcsec}^{-2}\right)$ & $-0.894 \pm 0.061$ & $2.6 \pm 0.3$ & $0.991 \pm 0.737$ & $0.090_{-0.063}^{+0.110}$ & $-0.830 \pm 0.073$ & $3.3 \pm 0.4$ \\
\hline$r_{\mathrm{c}} /\left(r_{500} 10^{-2}\right)$ & $0.932 \pm 0.060$ & $1.6 \pm 0.2$ & $0.378 \pm 0.068$ & $0.63 \pm 0.06$ & $0.698 \pm 0.085$ & $2.8 \pm 0.5$ \\
\hline$n_{0} /\left(10^{-2} h_{71}^{1 / 2} \mathrm{~cm}^{-3}\right)$ & $-1.194 \pm 0.051$ & $3.5 \pm 0.2$ & $-0.860 \pm 0.151$ & $1.8 \pm 0.4$ & $-1.016 \pm 0.046$ & $4.1 \pm 0.2$ \\
\hline$K_{\mathrm{BIAS}} /\left(100 h_{71}^{-1 / 3} \mathrm{keV} \mathrm{cm}^{2}\right)$ & $0.975 \pm 0.062$ & $4.7 \pm 1.2$ & $0.501 \pm 0.081$ & $1.5 \pm 0.3$ & $1.314 \pm 0.166$ & $4.4 \pm 3.3$ \\
\hline $\exp \left[\text { Cooling Radius } /\left(10^{-2} r_{\mathrm{vir}}\right)\right]^{\dagger}$ & $-0.350 \pm 0.025$ & $11.1 \pm 1.1$ & $-0.584 \pm 1.055$ & $70.46_{-70.45}^{+653.06}$ & $-0.336 \pm 0.026$ & $12.2 \pm 1.0$ \\
\hline$\left(\dot{M}_{\text {Spec }} / M_{500}\right) /\left(10^{-14} h_{71} \mathrm{yr}^{-1}\right)$ & $-0.598 \pm 0.121$ & $1.5 \pm 0.4$ & $-0.996 \pm 1.313$ & $0.78 \pm 0.33$ & $-0.404 \pm 0.163$ & $4.0 \pm 0.9$ \\
\hline$\left(\dot{M}_{\text {Classical }} / M_{500}\right) /\left(10^{-14} h_{71}^{-1} \mathrm{yr}^{-1}\right)$ & $-0.816 \pm 0.742$ & $1.1_{-10}^{+2.3}$ & $0.966 \pm 0.675$ & $0.018_{-0.016}^{+0.040}$ & $-0.280 \pm 0.163$ & $4.1 \pm 1.3$ \\
\hline $\exp [\alpha]^{\dagger}$ & $-3.726 \pm 0.198$ & $34 \pm 6$ & $-1.610 \pm 0.268$ & $2.8 \pm 0.9$ & $-3.589 \pm 0.345$ & $35 \pm 7$ \\
\hline$L_{\mathrm{X}} /\left(10^{32} h_{71}^{1 / 2} \mathrm{ergs} \mathrm{s}^{-1} \mathrm{keV}^{-1} M_{\odot}^{-1}\right)$ & $-3.057 \pm 0.250$ & $0.41 \pm 0.07$ & $-1.114 \pm 0.167$ & $0.43 \pm 0.04$ & $-3.795 \pm 0.699$ & $0.24 \pm 0.14$ \\
\hline $\exp \left[T_{0} / T_{\mathrm{vir}}\right]^{\dagger}$ & $4.931 \pm 0.365$ & $0.043 \pm 0.013$ & $-0.102 \pm 0.290$ & $0.76 \pm 0.10$ & $5.047 \pm 2.421$ & $0.041_{-0.037}^{+0.099}$ \\
\hline $\exp [\text { Slope of } T \text {-profile }]^{\dagger}$ & $6.908 \pm 1.209$ & $5.2 \pm 1.0$ & $-1.208 \pm 0.271$ & $0.32 \pm 0.04$ & $17.48 \pm 16.60$ & $8.4 \pm 4.2$ \\
\hline $\exp \left[T_{0}(0.5-2.0 \mathrm{keV}) / T_{0}(2.0-7.0 \mathrm{keV})\right]^{\dagger}$ & $-3.469 \pm 3.389$ & $28_{-26}^{+75}$ & $-1.027 \pm 0.586$ & $1.0 \pm 0.5$ & $-3.957 \pm 2.316$ & $125_{-64}^{+215}$ \\
\hline$M_{\mathrm{gas}} /\left(10^{-3} h_{71}^{-3 / 2} M_{500}\right)$ & $-1.997 \pm 0.136$ & $0.58 \pm 0.10$ & $0.894 \pm 0.229$ & $0.49 \pm 0.06$ & $-1.832 \pm 0.171$ & $0.93 \pm 0.20$ \\
\hline$\left(\dot{M}_{\mathrm{Spec} 2} / M_{500}\right) /\left(10^{-14} h_{71} \mathrm{yr}^{-1}\right)$ & $-0.375 \pm 0.028$ & $3.3 \pm 0.4$ & $-0.708 \pm 0.248$ & $4.3 \pm 3.5$ & $-0.347 \pm 0.058$ & $6.3 \pm 0.8$ \\
\hline$\Sigma_{0} /\left(\mathrm{kT} 0^{-7}\right.$ photons $\left.\mathrm{cm}^{-2} \mathrm{~s}^{-1} \mathrm{keV}^{-1}\right)$ & $-0.892 \pm 0.039$ & $5.5 \pm 0.4$ & $-0.630 \pm 0.221$ & $2.5 \pm 1.6$ & $-0.914 \pm 0.063$ & $5.7 \pm 0.4$ \\
\hline$M_{\mathrm{gas}} /\left(10^{-4} k T M_{500} \mathrm{keV}^{-1}\right)$ & $-1.654 \pm 0.089$ & $2.9 \pm 0.2$ & $-0.805 \pm 0.138$ & $1.02 \pm 0.17$ & $-1.458 \pm 0.127$ & $3.6 \pm 0.4$ \\
\hline
\end{tabular}

Notes. Columns: (1) the parameter name; (2) the fitted slope $(\Gamma)$ including all clusters; $(3)$ the fitted normalization $\left(\mathrm{CCT}_{0}\right)$ including all clusters; (4) $\Gamma$ for the SCC clusters; (5) $\mathrm{CCT}_{0}$ for the SCC clusters; (6) $\Gamma$ for WCC and NCC clusters and (7) $\mathrm{CCT}_{0}$ for the WCC and NCC clusters. ${ }^{\dagger}$ For this parameter the fitting function was $\mathrm{CCT}=\mathrm{CCT}_{0}(\exp [\text { parameter }])^{\Gamma}$.

Unfortunately, when compared to $n_{0}$ and $K_{0}$, most of the other parameters show a lot of scatter in their relation with CCT. Of those other parameters $K_{\text {BIAS }}$ appears to show the tightest correlation. Unlike $K_{0}, K_{\mathrm{BIAS}}$ is not dependent on $n_{0}$, but on the average density in a somewhat larger region. It is therefore not limited by the need to resolve the central region. There are, however, two problems with using $K_{\mathrm{BIAS}}$ as a proxy: (1) it requires a large enough region to fit a spectrum (which can be physically quite large for distant clusters) and (2) the size of the region from which $K_{\text {BIAS }}$ is determined differs between observations (i.e. it is dependent mainly on observing time and redshift). As a solution to (1) we suggest that the normalization of the thermal model is not as difficult to constrain as the temperature or metalicity (not as many counts are needed). In extreme cases the temperature and metalicity could be frozen at their overall cluster values so that only the central normalization would need to be constrained. As a solution to (2) we suggest that the region size be defined such that it is only dependent on the density profile and is independent of redshift, temperature and observing time. Specifically if two clusters have identical density profiles, then $K_{\text {BIAS }}$ should be derived from physical regions of the same size.

We suggest using a constant value of the projected emission measure in a cylinder,

$I(R) \equiv 2 \pi \int_{-\infty}^{\infty} \int_{0}^{R} n_{\mathrm{e}} n_{\mathrm{H}} r \mathrm{~d} r \mathrm{~d} l$

to determine region sizes that depend only on the density profile. Here we define $n_{\mathrm{e}}$ as the electron density and $n_{\mathrm{H}}$ as the proton density. One can simply use the density profile or model for a cluster to directly determine $R$ for a predetermined value of $I$. Alternately, for given spectral parameters and distance, the ratio of the count rate to normalization (as defined in XSPEC for example) is determined, so that the total observed count, $C$, can be determined and used to define $R$. That is, the count threshold for a region of size $R$ that has a given value of $I$ is:

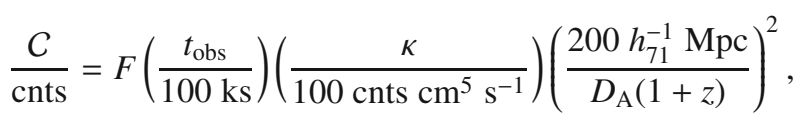

where $t_{\mathrm{obs}}$ is length of the observation, $\kappa$ is the ratio of the count rate to normalization and $F$ is a constant that depends on the desired value of $I$,

$F \equiv \frac{10^{-11}}{16 \pi}\left(\frac{I}{h_{71}^{-2} \mathrm{Mpc}^{-2} \mathrm{~cm}^{-1}}\right)$

A value of $I=8.6 \times 10^{65} h_{71}^{-2} \mathrm{~cm}^{-3}$, corresponding to $F=17910$, produces a 10000 count region (in the $0.5-7.0 \mathrm{keV}$ band) for a metalicity of $Z=0.25$, a photoelectric absorption column density of $N_{H}=2 \times 10^{20} \mathrm{~cm}^{-2}$ and our median virial temperature (4.3 keV), redshift (0.047) and observing time time (44 ks). See Appendix B for more details on these calculations.

Although this method makes the $K_{\text {BIAS }}$ region size consistent among clusters, there is an additional problem that a reasonable value of $I$ at one redshift will often be unreasonable for clusters at a significantly different redshift. For instance, for our proposed value of $I=8.6 \times 10^{65} h_{71}^{-2} \mathrm{~cm}^{-3}$, which leads to a 10000 count region for our median cluster parameters, would produce a 58 count region for a cluster with identical parameters but at a redshift of $z=0.5$. However, if the region size is not constant for all studies (say the chosen value of $I$ varies depending on the study), it makes comparisons between studies difficult.

After $K_{\text {BIAS }}$, the next best parameter appears to be the scaled central X-ray luminosity ( scaled $L_{X}$ ). The region from which we extracted scaled $L_{X}$ is well-defined $\left(0-0.048 r_{500}\right)$, but suffers from two other problems. The scaling depends on $M_{\text {gas }}$, which in turn depends on $n_{0}$. In addition, as with $K_{\text {BIAS }}$, a spectrum is needed in order to determine the luminosity. Of course, as with $K_{\text {BIAS }}$, in extreme cases the overall cluster temperature and metalicity can be used when getting an estimate of the core 
luminosity. The major advantage in using scaled $L_{\mathrm{X}}$ over $K_{\mathrm{BIAS}}$ is that it is better defined for this study.

For distant clusters in which only a few thousand counts are available, it is necessary to use $\Sigma_{0}$ or $\alpha . \Sigma_{0}$ has the advantage that surface brightness profiles only need $\sim 50$ counts per bin, as opposed to spectra that require thousands of counts. Unfortunately there is the problem of resolution. For very distant clusters, it may not be possible to resolve $\Sigma_{0}$ in the very central regions. Also, generally if $\Sigma_{0}$ is measurable then $n_{0}$ is also measurable and is a much better proxy. However, if $\Sigma_{0}$ is the only parameter available, it should be divided by $k T_{\text {vir }}$ before being used as a proxy (Fig. 7). Cutting $\Sigma_{0} / k T_{\text {vir }}$ at $\sim 1.5 \times 10^{-7}$ counts $\mathrm{cm}^{-2} \mathrm{~s}^{-1} \mathrm{keV}^{-1}$ does a good job of separating NCC and CC clusters.

This leaves cuspiness as the only viable candidate for distant clusters with few counts. Unfortunately, there is a great deal of scatter in the $\alpha$-CCT plot. Moreover, there seems to be a drop in $\alpha$ at CCT $\sim 1 h_{71}^{-1 / 2} \mathrm{Gyr}$, so that SCC clusters with $\mathrm{CCT} \sim 1 h_{71}^{-1 / 2}$ Gyr have the same value of $\alpha$ as the WCC clusters. Unlike $\Sigma_{0}$ there does not seem to be any trend with $T_{\text {vir }}$ and we have not found any parameter which can be used to tighten this correlation. Cutting $\alpha$ at 0.75 ensures for the most part that the sample above the cut will be CC clusters, although not necessarily SCC clusters as suggested by Vikhlinin et al. (2007). Cutting $\alpha$ at 0.5 seems to capture most of the CC clusters above the cut, with a slight contamination from the steepest NCC clusters. Finally cutting at $\alpha=1$ ensures that the clusters above the cut are SCC clusters, although there are still several SCC clusters below the cut. In short, it is impossible to separate out a sample, but it is safe to say a cluster with $\alpha>1$ is an SCC cluster and a cluster with $\alpha>0.5$ is most likely a CC cluster.

Recently, Santos et al. (2008) suggested a concentration parameter $\left(C_{\mathrm{SB}}\right)$ as a proxy for determining whether a distant cluster is a CC or not (see Eq. (2)). Although this proxy is beyond the scope of our analysis (the residual background must be carefully handled to find the surface brightness at $400 \mathrm{kpc}$ ), we used our $\beta$ model fits to estimate $C_{\mathrm{SB}}$. Figure 9 shows the results of the fit, which seems very promising. The one strong outlier is NGC 1399. As discussed in Appendix C.10, the surface brightness profile of the Fornax cluster shows a flattening at $\sim 0.04-0.05 r_{500}$, probably due to NGC 1404. This flattening will cause a severe overestimate of $\Sigma(r<400 \mathrm{kpc})$ when extrapolating the profile out to $400 \mathrm{kpc}$. To do a true comparison, we would need to measure the integrated surface brightness, rather than use models extrapolated from $\sim 0.05 r_{500}$.

\section{Discussion}

\subsection{The cooling flow problem}

The cooling flow problem is the discrepancy between the spectrally determined mass deposition rate and the classically determined mass deposition rate. Figure 10 clearly demonstrates the cooling flow discrepancy. In all but three of the 46 CC clusters, the spectral mass deposition rate is less than the classical mass deposition rate, usually by at least one order of magnitude (dashdot line).

In two of these three cases, NGC 1399 and A3266, the mass deposition rates are consistent within errors. Only in the case of A2634 is $\dot{M}_{\text {spec }}>\dot{M}_{\text {classical }}$. As noted in Sect. 3.3.7, all three of these clusters (A2634, NGC 1399 and A3266) have anomalously low values of $\dot{M}_{\text {classical }}$. The WCC clusters A2634 and A3266 appear to be involved in mergers (although as discussed later, probably not major mergers), the low values of $\dot{M}_{\text {classical }}$ being then

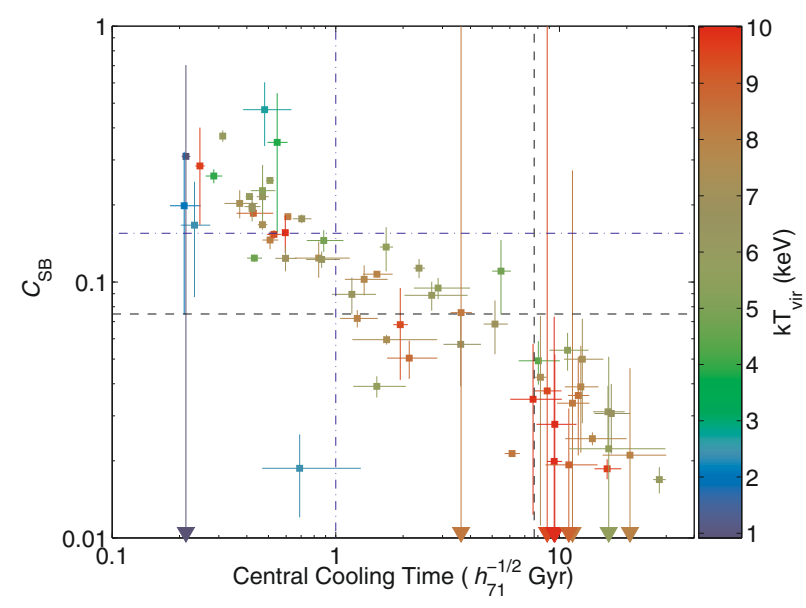

Fig. 9. The concentration parameter $C_{\mathrm{SB}}$ suggested by Santos et al. (2008) versus CCT. These preliminary results seem very promising. As discussed in the text, the outlier (Fornax cluster) is probably due to a problem in extrapolating the surface brightness profile. The horizontal blue dash-dot line and black dashed line show the suggested cuts of Santos et al. (2008) between SCC and WCC clusters (0.155) and WCC and NCC clusters (0.075), respectively.

consistent with a disrupted core. Also as noted in Sect. 3.2, $\dot{M}_{\text {spec }}$ can be over-estimated in merging clusters. Moreover in the case of A3266, it is on the line between NCC and WCC clusters with CCT $\sim 7.7 h_{71}^{-1 / 2}$ Gyr. As seen in Table 3, several of the NCC clusters have nonzero values of $\dot{M}_{\text {spec }}$, so it is not so surprising that A3266 would have $\dot{M}_{\text {spec }} \sim \dot{M}_{\text {classical }}$. The case of NGC 1399 is not as clear. In many of the plots NGC 1399 is an outlier. As discussed in Appendix C.10, this may be due to a problem with extrapolating the surface brightness profile. Even if this is the case, $\dot{M}_{\text {classical }}$ is small $\left(<1 h_{71}^{-2} M_{\odot} \mathrm{yr}^{-1}\right)$ and $\dot{M}_{\text {classical }} \approx \dot{M}_{\text {spec }}$ (see Appendix C.10 for details). The anomalously low $\dot{M}_{\text {classical }}$ indicates that the density drops off quickly. In fact the cooling radius is only $0.026 r_{500}$, which is among the four smallest. The other three (in order from smallest to largest) are A3266, A3667, A2634, all WCC merging clusters, two of which are also outliers in this plot. The SCC cluster with the next smallest cooling radius is A0262 that has a cooling radius of almost twice that of NGC 1399. Although NGC 1399 shows some evidence of merging (e.g. Drinkwater et al. 2001), it appears to be just starting to merge so only the outer regions have been affected. It is also possible that the galaxy NGC 1404 has disturbed the core as it fell in. A more interesting scenario is that since NGC 1399 is dynamically young (Y. Schuberth, private communication), the cooling flow has just formed so no energy has been injected yet and $\dot{M}_{\text {spec }} \approx \dot{M}_{\text {classical }}$.

With the exception of these three discrepancies, in the other 43 CC clusters, $\dot{M}_{\text {spec }} \ll \dot{M}_{\text {classical }}$. Assuming no energy is input to the $\mathrm{CF}$ and the $\mathrm{CF}$ formed $\sim 7.7 h_{71}^{-1 / 2} \mathrm{Gyr}$ ago, these two quantities should be equal. If the latter assumption is incorrect, we can find the cooling radius such that $\dot{M}_{\text {classical }}=\dot{M}_{\text {spec }}$. We define the cooling time at this radius to be the $\mathrm{CC}$ formation time (CCFT). We note that the CCFT is an upper limit to the actual time since formation because, firstly, as the gas flows to the center, its density increases causing it to cool more rapidly. Therefore the actual cooling time of the gas is shorter than $t_{\text {cool }}$, as defined in Eq. (14). Secondly, the spectroscopic mass deposition model, MKCFLOW, used to fit the line emission from a multiphase gas often provides a better fit than a single-temperature thermal model. In other words, it may find 


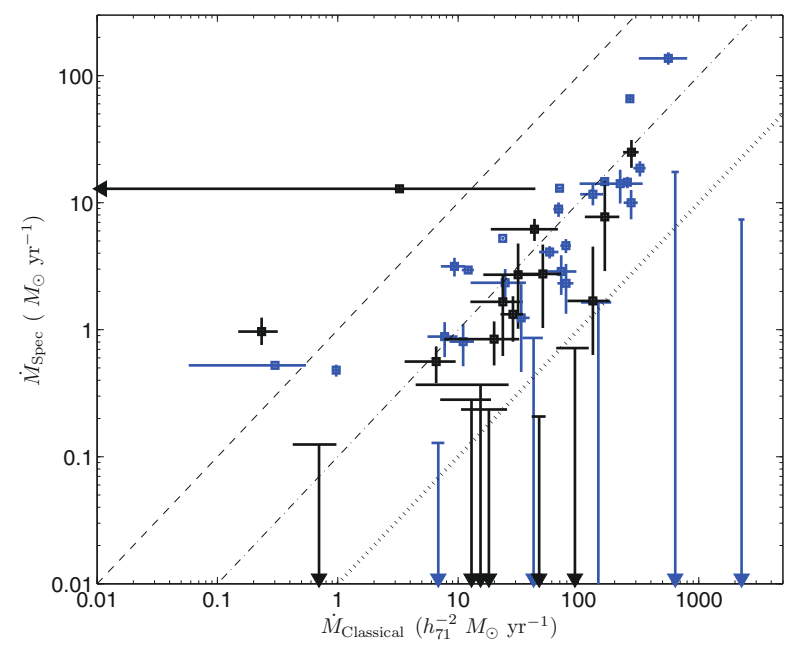

Fig. 10. Spectroscopic cooling rate $\dot{M}_{\text {spec }}$ versus classical cooling rate $\dot{M}_{\text {classical }}$ for the $46 \mathrm{CC}$ clusters. The dashed-line shows parity between the two. The dash-dot line and the dotted line show one and two orders of magnitude difference respectively. In all but three cases, $\dot{M}_{\text {spec }} \ll$ $\dot{M}_{\text {classical }}$.

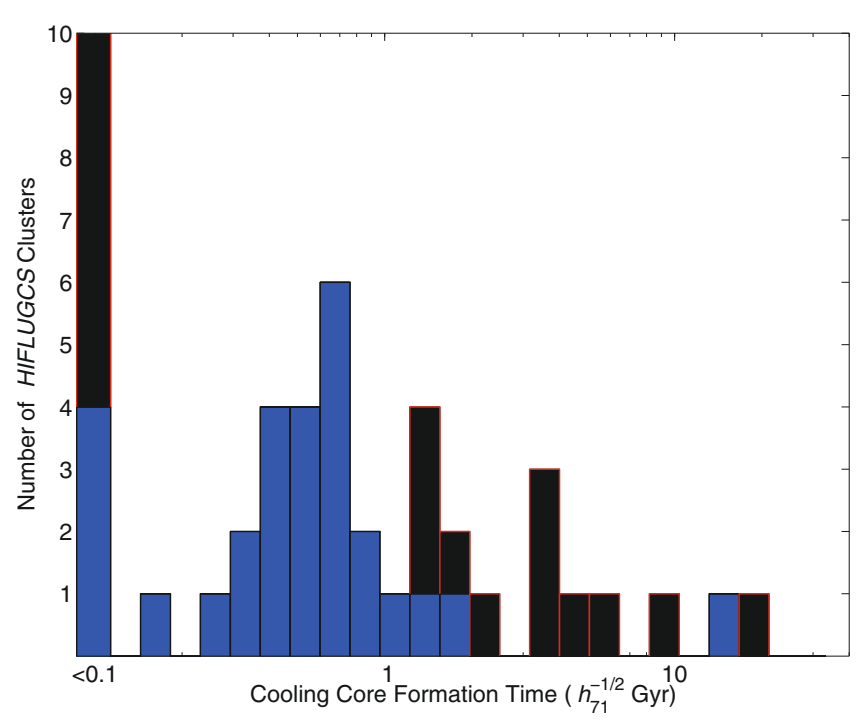

Fig. 11. This figure shows the histogram of cool core formation time (CCFT) for the $46 \mathrm{CC}$ clusters. Ten of the clusters only have upper limits for $\dot{M}_{\text {spec }}$. For there to be no discrepancy between $\dot{M}_{\text {classical }}$ and $\dot{M}_{\text {spec }}$, cooling flows would have to have formed very recently.

a cool gas component even when there is none, yielding $\dot{M}_{\text {spec }}$ to be greater than its actual value. Figure 11 shows the histogram of CCFT for the 46 CC clusters. Ten of the clusters have only upper limits for $\dot{M}_{\text {spec }}$, for the others the distribution peaks at around $0.5 \mathrm{Gyr}$, about five to ten times shorter than Allen et al. (2001a) found using ASCA and ROSAT data and assuming intrinsic absorption. This means in order for the observations to match, in the absence of heating, the CFs would have had to form very recently.

In order to check the plausibility that the cooling flow discrepancy is due to the assumption that cooling flows have formed very recently, we devised a rough test to estimate this likelihood. This test is based on the hypothesis that low- and high- $z$ clusters are both drawn from the same underlying population of clusters. Hence, the high- $z$ clusters in our sample, when evolved uninterrupted to allow their cool cores to grow, should have mass

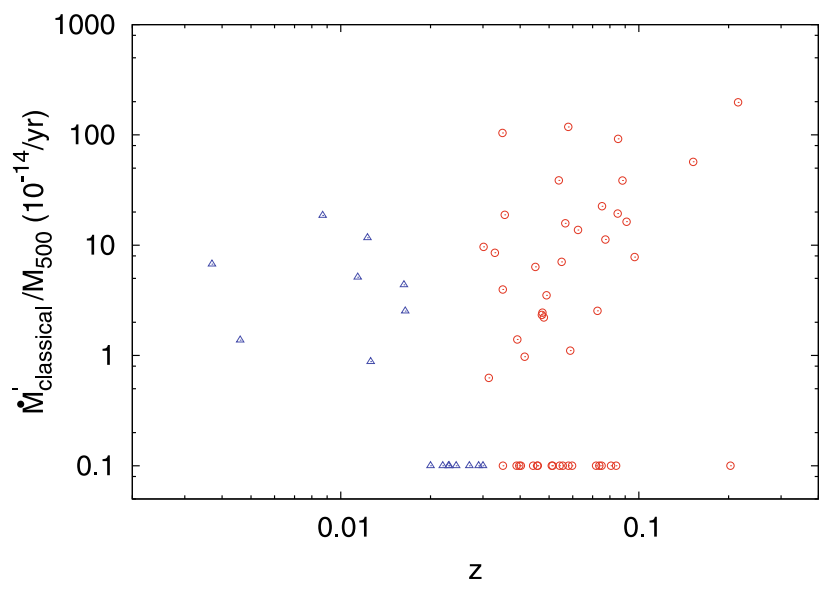

Fig. 12. This figure shows the forward-evolved $\dot{M}_{\text {classical }}$ for high- $z$ clusters in redshift bins 2, 3 and 4 (red open circles) and original $\dot{M}_{\text {classical }}$ for low- $z$ clusters in redshift bin 1 (blue open triangles).

deposition rates comparable to the low- $z$ clusters in our sample. We show below quantitatively, for the first time, that the likelihood of this hypothesis is very small.

There are two major limitations to this very simple test. Firstly, our sample is flux-limited and hence due to selection effects we are likely to pick clusters of increasing mass with increasing redshift. Secondly, even though our sample spans a limited redshift range, the cluster population changes with redshift. This is because, in a hierarchical Universe, the more distant clusters (high-mass) will actually never evolve into the mostly low-mass clusters we observe locally. These two reasons together make a comparison of properties between low- $z$ and high$z$ clusters difficult. As an approximate solution to the first problem, we scaled the mass deposition rates by $M_{500}$, even though this causes a bias against the high- $z$ clusters. This is due to the fact that at a fixed gas density, the cooling time is an increasing function of the temperature, CCT $\propto T^{1 / 2} \propto M^{1 / 3}$. Thus, if clusters are self-similar, we should expect the cooling rate to scale with mass more slowly than $\propto M$. Consequently, under the assumption that the cooling flows are recent phenomena, we should not expect the high mass clusters evolved to the lookback time of low- $z$ clusters to show higher $\dot{M}_{\text {classical }} / M_{500}$ than the lower mass ones.

We divided our sample into four redshift bins, each with 16 clusters. Although we have a local sample (median redshift for the 16 most distant clusters is $z \sim 0.08$ ), most of our clusters span a lookback time, $t_{\text {lookback }} \sim 1 h_{71}^{-1 / 2} \mathrm{Gyr}^{17}>\langle C C F T\rangle$. We forward evolved each cluster in redshift bins 2,3 and 4 by adding to its CC formation time, $C C F T$, the difference between its lookback time, $L B T$, and the mean lookback time of clusters in the first bin, $\langle L B T\rangle_{1}$. Hence,

$$
C C F T^{\prime}=C C F T+L B T-\langle L B T\rangle_{1},
$$

where $C C F T^{\prime}$ is the forward-evolved CCFT of that cluster. In order to calculate the cooling rate in these forward-evolved systems, we determined the radius, $R^{\prime}$, at which the cooling time of the gas equals $C C F T^{\prime}$ and calculated $\dot{M}_{\text {classical }}$ at this radius, $\dot{M}_{\text {classical }}^{\prime}$. Shown in Fig. 12 is $\dot{M}_{\text {classical }}^{\prime}$ scaled by $M_{500}$ versus redshift. Since the low- $z$ clusters belonging to redshift bin 1 (blue open triangles) are not evolved forward in time, these have $\dot{M}_{\text {classical }}^{\prime}=\dot{M}_{\text {classical }}$. Despite the fact, as noted above, that scaling $\dot{M}_{\text {classical }}^{\prime}$ by $M_{500}$ very likely overcompensates the

17 This is the lookback time from $z=0$ to $z \sim 0.08$. 
tailing-off of clusters due to selection effects, there seems to be a marked difference in the parent low $-z$ and high- $z$ (red open circles) samples.

Of the 10 clusters in the highest redshift bin with $\dot{M}_{\text {classical }}^{\prime}$ greater than zero, 8 have $\dot{M}_{\text {classical }}^{\prime} / M_{500}>10^{-13} \mathrm{yr}^{-1}$, while of the 8 clusters in the lowest redshift bin with $\dot{M}_{\text {classical }}^{\prime}$ greater than zero, only 2 have $\dot{M}_{\text {classical }}^{\prime} / M_{500}>10^{-13} \mathrm{yr}^{-1}$. If the two subsamples are drawn from the same distribution, the probability, $p$, of any one cluster having $\dot{M}_{\text {classical }}^{\prime} / M_{500}>10^{-13} \mathrm{yr}^{-1}$ should be the same for both. The joint probability, $P(p)=P\left(p ; m_{1} \leq\right.$ 2) $P\left(p ; m_{2} \geq 8\right)$, of drawing one sample of 8 with no more than 2 clusters in this category and a second sample of 10 with at least 8 , is maximized for $p=p_{\max }=0.5584$, giving $P\left(p_{\max }\right)=$ $P_{\max } \leq 0.00883$. Repeating this calculation for all possible pairs of draws (always using the smaller value for the first of the pair), if the true probability of $\dot{M}_{\text {classical }}^{\prime} / M_{500}>10^{-13} \mathrm{yr}^{-1}$ is $q$, the probabilities of each pair for which $P_{\max } \leq 0.00883$, can be summed to compute the likelihood of this outcome. This is maximized for $q=0.5$, giving 0.029 for the likelihood. Thus, the chance that both samples are drawn from the same population is no more than $2.9 \%$.

We also used the Kolmogorov-Smirnov (K-S) test to distinguish between the two samples. Even though it is well-known that the K-S method exhibits poor sensitivity to the deviations that occur in the tails of any given two distributions, we find the probability of the null hypothesis, i.e. the probability that the low $-z$ and the high- $z$ clusters are drawn from the same distribution, is only $\sim 2.4 \%$. From this we can conclude that the high- $z$ clusters, after having been evolved to match the physical state of low- $z$ clusters, have systematically higher $\dot{M}_{\text {classical }}$ than the low- $z$ clusters. Thus, it is very unlikely that the discrepancy between classical and spectral mass deposition rates in cool-core galaxy clusters can be explained away by invoking the recent cool-core formation hypothesis.

We note there are 27 clusters with zero $\dot{M}_{\text {classical }}^{\prime}$. Of these, 18 constitute the entire NCC cluster subsample, 6 are WCC clusters and 3 are SCC clusters. That the NCC clusters show no mass deposition rate even after evolving them forward is not surprising. Out of the $9 \mathrm{WCC}+\mathrm{SCC}$ clusters, the five high- $z$ clusters are ones with extremely short or zero $C C F T$ and evolving them forward still results in $C C F T^{\prime}$ to be shorter than the cooling time at $R=0$.

Another interesting exercise is to check the emission measure distribution of the cool gas. In terms of the power radiated per unit temperature, Peterson et al. (2003) parametrized this as:

$$
\frac{\mathrm{d} L}{\mathrm{~d} T}=\frac{5}{2} \frac{\dot{M} k}{\mu m_{p}}(\delta+1)\left(\frac{T}{T_{0}}\right)^{\delta} .
$$

For a steady cooling flow at constant pressure, we would have $\delta=0$, but if cooling is retarded then the amount of gas at lower temperatures is reduced $(\delta>0)$. With our limited spectral resolution we approximate the fit in the following way. We assume that $\dot{M}_{\text {spec2 }}$ should be compared to the differential luminosity $\left(\frac{\mathrm{d} L}{\mathrm{~d} T}\right)$ between the two fitted temperatures $k T_{\text {high }}\left(T_{0} \equiv T_{\text {high }}\right)$ and $k T_{\text {low }}$. We assume that $\dot{M}_{\text {spec }}$ should be compared to $\frac{\mathrm{d} L}{\mathrm{~d} T}$ between $k T_{\text {low }}$ and zero. Our argument for using $k T_{\text {low }}$ and not $k T_{\text {high }}$ is that the small values of $\dot{M}_{\text {spec }}$ (compared to $\dot{M}_{\text {classical }}$ and especially $\dot{M}_{\text {spec2 }}$ ) are due to the lack of lines for the coolest gas and therefore it gives a better estimation of the true mass deposition between $k T_{\text {low }}$ and zero than between $k T_{\text {high }}$ and zero. The fraction of observed gas is $\dot{M}_{\text {spec } 2} / \dot{M}_{\text {classical }}$ and $\dot{M}_{\text {spec }} / \dot{M}_{\text {classical }}$ respectively. Figure 13 is similar to Fig. 7 in Peterson et al. (2003).

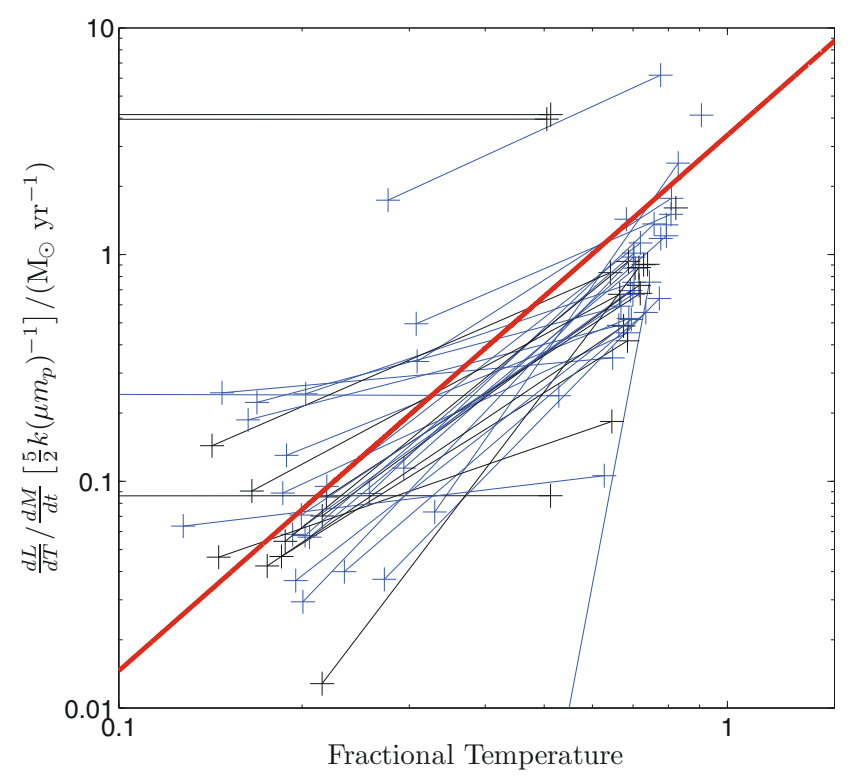

Fig. 13. The fraction of differential luminosity vs. $T_{0} / T_{\text {vir }}$, similar to Fig. 7 in Peterson et al. (2003). The weighted average value of $\delta$ (see text) for the clusters with measurements at both data points is $\sim 2.0$ and is shown as the thick red-line. The blue crosses and lines are SCC clusters and the black are WCC clusters.

For each cluster with measurements at both points (i.e. neither was an upper-limit) we did a fit to Eq. (24). Our rough estimation of $\delta$, based on the weighted average value of the fits, is $\sim 2.0$ (with a very large variance). This value is on the steep end of the values found by Peterson et al. (2003). One may think this slight difference is due to the fact that we are working with an unbiased sample and their sample was picked to be SCCs (in general), so that $\dot{M}_{\text {spec }}$ is not far from $\dot{M}_{\text {classical }}$. However, there does not seem to be a separation of the SCC clusters (blue) and the WCC clusters (black). The three outliers in the upper left are: NGC 1399, A3266 and A2634, which as mentioned earlier have $\dot{M}_{\text {spec }} \gtrsim \dot{M}_{\text {classical }}$. This model requires further study with higher spectral resolution instruments before any definite conclusion can be drawn, but it is reassuring that we find consistent results using a complete, unbiased sample of clusters that is more than four times larger.

\subsection{Temperature profiles}

Perhaps two of the most striking plots from Fig. 6 are subplots $J$ and $K$, which show a sudden break at $\sim 1 h_{71}^{-1 / 2}$ Gyr. In fact, we find that for the most part only SCC clusters have a central temperature drop. All 28 SCC clusters have a central temperature drop and of the eight non-SCC clusters with a central temperature drop (see Table 3), four (A1650, A2065, A2142 and A2589) have CCT $<2 h_{71}^{-1 / 2}$ Gyr, putting them on the border between SCC and WCC clusters. Additionally two (A2142 and A3667) have well-known cold fronts. The other three appear to be merging clusters in which a cool core has survived: A0400 (Hudson et al. 2006), A0576 (Dupke et al. 2007b) and A2256 (Sun et al. 2002). It is also clear from Fig. 6K that the inner temperature profiles of these eight non-SCC clusters are, for the most part, flatter than those of the SCC clusters (slope $\gtrsim-0.2$ ). We argue that based on this fact, studies such as those of Burns et al. (2008) and Sanderson et al. (2006a), which define CC clusters as clusters with a central temperature drop, are basically 


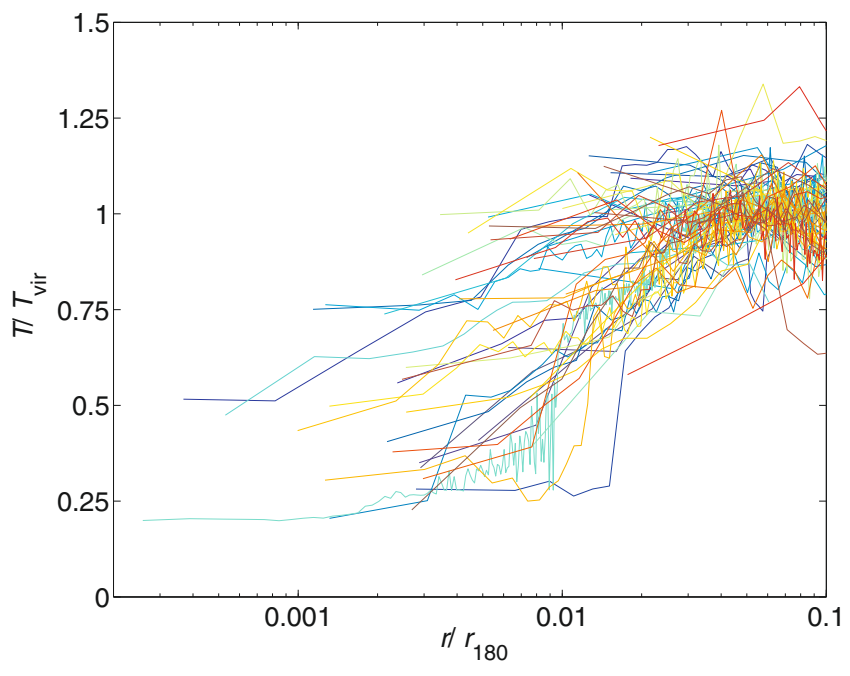

Fig. 14. The inner temperature profiles of all 64 HIFLUGCS clusters scaled by virial temperature and radius. The error bars on the measurements have been omitted for clarity. There appears to be a range of slopes rather than a bimodal distribution.

defining CC clusters as clusters with CCT $<1 h_{71}^{-1 / 2} \mathrm{Gyr}$ (SCC clusters in our sample).

Some authors claim the existence of a universal inner temperature profile for relaxed clusters (e.g. Allen et al. 2001b; Sanderson et al. 2006b), while others find no such universality (e.g. Vikhlinin et al. 2005). A universal inner temperature profile suggests that clusters either have a flat central temperature profile or a drop that scales with $T_{\text {vir }}$ and $r_{\text {vir }}$. It is clear from Fig. 4L that we do not see such a universal inner temperature profile. Figure 14 shows all 64 of our temperature profiles out to $0.1 r_{\text {vir }}$, scaled by $r_{\text {vir }}$ and $T_{\text {vir. }}$. It is clear from this figure why we do not see a bimodal distribution in Fig. 4L. There is a continuous range of slopes from a $20 \%$ increase above $T_{\text {vir }}$ (MKW8) to a decrease down to $0.2 T_{\text {vir }}$ (A3526 - Centaurus Cluster). We note that some authors (e.g. Sanderson et al. 2006a) only use single thermal models at all radii when constructing temperature profiles. We, however, used double thermal models for the inner annuli in 14 of our SCC clusters since the addition of the second thermal component improved the fit significantly ( $>99 \%$ confidence according to the $F$ test). In these cases the lower temperature was used, causing a steeper temperature profile than if a single thermal model had been used.

It is interesting to note that in Fig. $4 \mathrm{~K}$, there does seem to be a bimodal distribution in $T_{0} / T_{\text {vir }}$ (albeit with a large dispersion). This suggests that while the radius of the gas with $T<T_{\text {vir }}$ does not scale with cluster size, the depth of the drop does. SCC clusters have a central temperature of $\sim 0.4 T_{\text {vir }}$, whereas NCC and WCC clusters have $T_{0} \sim T_{\text {vir }}$. As we discuss in the next section, it is unclear whether this cool gas is associated with the CF or the central galaxy. In the case of the former this indicates the coolest major component of gas allowed by the feedback mechanism. That is, significant quantities of gas do not cool below $\sim 0.4 T_{\text {vir. }}$. In the case of the latter, it is consistent with observing that the mass of the brightest cluster galaxy (BCG) scales with the cluster mass, so that $T_{0} / T_{\text {vir }}$ is constant. This model would support the simulations of Burns et al. (2008) who find that the $\mathrm{CC}$ grows with the cluster.

\subsubsection{What causes the central temperature drop?}

An old but still ongoing controversy is whether the temperature drop in CC clusters is related to the CF or is simply a reflection of the potential well around the BCG. In the case of the former, the central temperature should be reflected by a modified cooling flow model and should simply depend on the energy factors (energy radiated away, along with input energy from conduction and any feedback mechanism). In the latter case the temperature should reflect the mass of the central galaxy and should be independent of any cooling flow model.

One check for possible multitemperature components is the need for a double thermal model in the central annuli. In the central annulus (annuli) for 14 of 64 HIFLUGCS clusters (all SCC clusters), a double thermal fit was a significantly better fit than a single thermal model. It is possible, since these are projected temperature profiles, this is simply due to many temperature components along the line of sight. Even in this case, the projected temperature components that dominate are the ones near the cluster center where the emission is peaked. Whether it is the projected spectra of outer annuli or truly the need for a double temperature fit to the central spectra, we take this as evidence of many temperature components in the region of the emission peak for these clusters. Thus the change in temperature is rapid in these regions. To explicitly demonstrate that a double thermal model is consistent with more than two temperature components we ran simulations of spectra with four and eight thermal models with temperatures equally spaced between 1 and $2 \mathrm{keV}$. Fitting these spectra indicates that a double thermal model can, at Chandra's energy resolution, fully describe the plasma emission of the underlying four- or eight- temperature components and provides the statistically best fit to the spectrum $\left(\chi^{2} / \text { d.o.f. } \sim 1\right)^{18}$. For the eight temperature plasma the returned temperatures were: $1.80 \pm 0.08 \mathrm{keV}$ and $1.26 \pm 0.03 \mathrm{keV}$. Therefore we interpret annuli that require two thermal models to contain, at minimum two thermal components, with a low temperature component $\lesssim T_{0}$.

Figure 15 shows the modified spectral mass deposition rate, $\dot{M}_{\text {spec2 }}$ versus $\dot{M}_{\text {classical }}$. The striking thing about this plot is that the two values are very often consistent with each other, especially for the SCC clusters. Based on this result, one may naïvely believe that the gas cools to $T_{\text {low }}$ (the lowest temperature for the modified cooling flow model - see Sect. 2.8) at the rate predicted by the classical cooling flow model, but does not cool at a significant rate below this temperature. If this is the case there are two possibilities: (1) the gas is in thermal equilibrium throughout this range of temperatures or (2) the gas is rapidly heated from $T_{\text {low }}$ to $T_{\text {high }}$, when it reaches $T_{\text {low }}$. An alternate explanation is that with the spectral resolution of the Chandra ACIS, we are unable to distinguish between gas cooling from $T_{\text {high }}$ to $T_{\text {low }}$ and a rapid drop of temperature at the center. That is, the observation that $\dot{M}_{\text {spec2 }} \approx \dot{M}_{\text {classical }}$ could simply be a coincidence.

Investigating further, we plotted $k T_{\text {low }}$ versus $k T_{0}$ (Fig. 16) for the CC clusters. The blue points are SCC clusters and the black points are WCC clusters. If the gas cools from $T_{\text {high }}$ to $T_{\text {low }}$ at a rate consistent with a classical cooling flow (and is somehow stopped at $T_{\text {low }}$ ), then we would expect $T_{\text {low }} \lesssim T_{0}$. Fig. 16 shows this to be the case for SCC clusters, however no

\footnotetext{
${ }_{18}$ For example, for eight thermal components, the average reduced $\chi^{2}$ for a single thermal fit was $1.46,0.976$ for two thermal components, and 0.984 for three thermal components. The simulations were done for a $100 \mathrm{ks}$ observation with the background and thermal normalization taken from A1795's central annulus. All thermal components were given the same input normalization.
} 


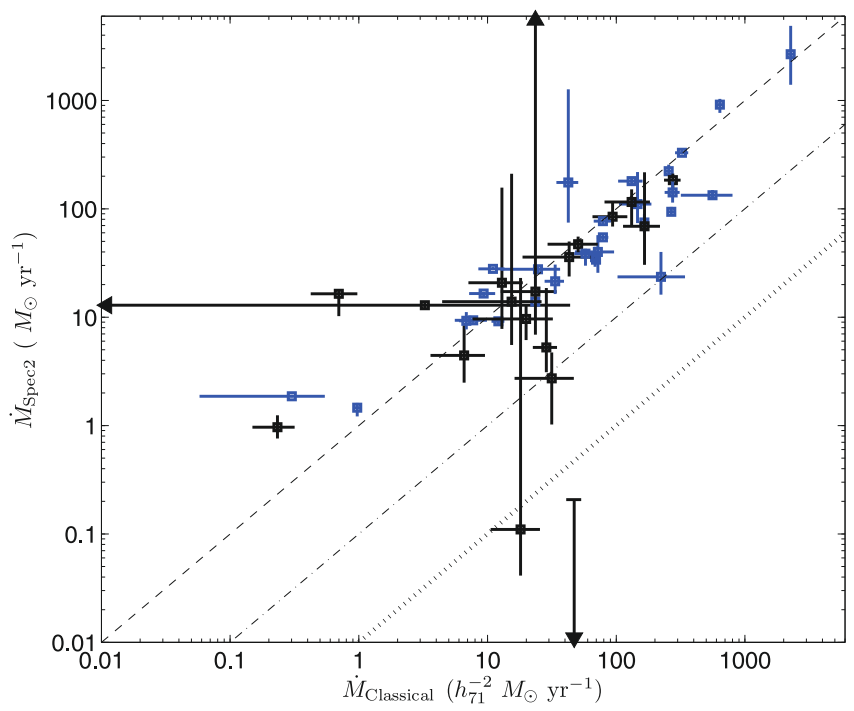

Fig. 15. This plot is similar to Fig. 10. $\dot{M}_{\text {spec2 } 2}$ is the spectrally determined mass deposition rate between $T_{\text {high }}$ and $T_{\text {low }}$, where the lower temperature is left free (unlike for $\dot{M}_{\text {spec }}$ ). The lines are the same as for Fig. 10. The mass deposition rates measured by both methods generally yield consistent results.

correlation is found for WCC clusters. As noted earlier, WCC clusters show little or no temperature drop at the cluster center. However, when fitted with a modified spectral mass deposition model, there appear to be significant quantities of gas down to $\sim 1 / 3 T_{\text {vir }}$. The simplest explanation is that $\dot{M}_{\text {spec2 }}$ is not the correct model and at the energy resolution of the Chandra ACIS, it is difficult to distinguish between multitemperature components along the line-of-sight and a cooling flow model down to $\sim 1 / 3 T_{\text {vir. }}$. With the spectral resolution of the Chandra ACIS we cannot draw any strong conclusions, however the results for the SCC clusters argue for further investigation with higher spectral resolution instruments to see if this trend of $T_{\text {low }} \lesssim T_{0}$ persists.

In order to check for the correlation of the central cluster gas with the central galaxy, we compared the position of the brightest cluster galaxy (BCG) to the position of the emission peak. We identified the BCGs from visual inspection of the $R$ band images of the Second-Epoch Digitized Sky survey (DSS2, see e.g. http: //archive.stsci .edu/dss) and then extracted magnitudes and redshifts from NED, HyperLeda ${ }^{19}$ (Paturel et al. 2003) or the compilation by Andernach \& Tago (see Andernach et al. 2005, for a description). We measured the position of the BCG by fitting a bidimensional Gaussian on its image, using the NRAO program FITSview ${ }^{20}$. The typical uncertainty was $\sim 0$ '.5. Visual inspection of these images allowed the identification of usually one, sometimes two or three BCG candidates. In the cases in which there was more than one BCG candidate, we used the candidate closest to the X-ray peak. Figure 17 shows the histogram of distance between the X-ray peak and BCG. The histogram is color coded: blue for SCC clusters, black for WCC clusters and red for NCC clusters. The fact that all SCC clusters have a BCG at the center seems to support the idea that the central temperature drop is related to the presence of the galaxy. On the other hand, $78 \%$ of all HIFLUGCS clusters have a BCG

19 http://leda.univ-lyon $1 . \mathrm{fr} /$

${ }^{20}$ http://www.nrao.edu/software/fitsview; the (USA) National Radio Astronomy Observatory (NRAO) is operated by Associated Universities, Inc. and is a Facility of the (USA) National Science Foundation.

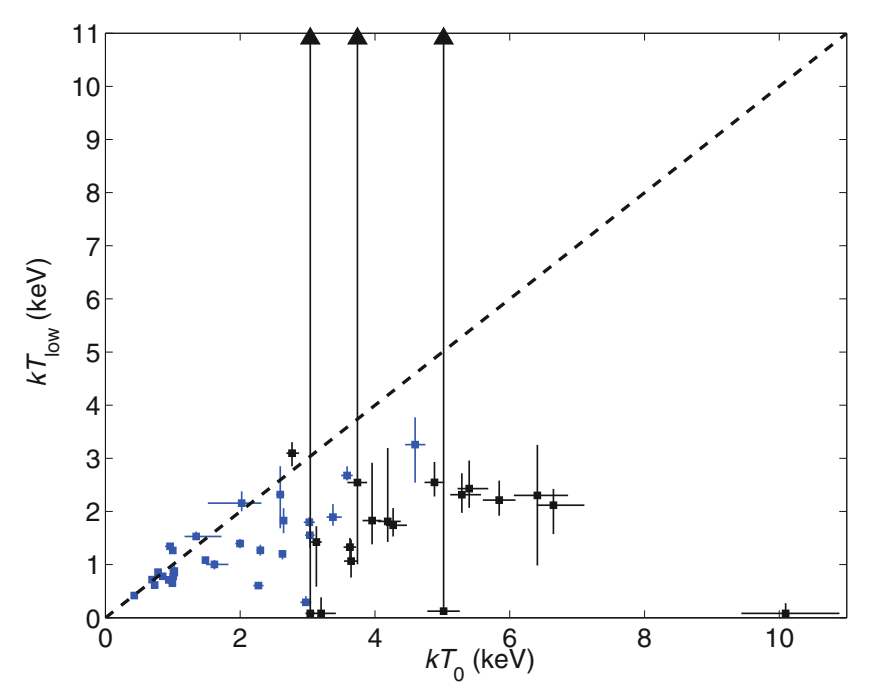

Fig. 16. The lower temperature in the modified cooling flow model $\left(k T_{\text {low }}\right)$ versus the cluster central temperature $\left(k T_{0}\right)$. Blue points are SCC clusters, while black points are WCC clusters. In general $T_{\text {low }}<T_{0}$, which is expected if there are multitemperature components between $T_{\text {high }}$ and $T_{\text {low }}$. The fact that the WCC clusters show a greater discrepancy between $T_{\text {low }}$ and $T_{0}$ suggests that at the energy resolution of Chandra's ACIS it is difficult to distinguish between the modified cooling flow model and multitemperature components along the line of sight. The three cases where $T_{\text {low }}$ is unconstrained, $\dot{M}_{\text {spec2 }}$ is consistent with zero.

within $12 h_{71}^{-1} \mathrm{kpc}$ of their X-ray peak (including $61 \%$ and $50 \%$ of WCC and NCC clusters respectively) and $~ 88 \%$ have a BCG within $50 h_{71}^{-1} \mathrm{kpc}$. There is a clear discrepancy between the number of WCC clusters $(92 \%)$ and NCC clusters $(61 \%)$ that have a BCG at the X-ray peak $\left(<50 h_{71}^{-1} \mathrm{kpc}\right)$ and those that have cool gas at their center: $33 \%$ and $11 \%$ respectively. Also as noted earlier the temperature drop in these clusters is generally smaller than it is for SCC clusters.

Here, we do not present this as evidence that temperature drops are or are not related to the potential of the BCG. On the one hand, all but one cluster (A2256) with a central temperature drop have a BCG cospatial with the X-ray peak $\left(<12 h_{71}^{-1} \mathrm{kpc}\right)$, suggesting that the temperature drop is related to the BCG. On the other hand, there are many clusters with a BCG at the center and no central temperature drop. It appears that regardless of the mechanism that causes the temperature of the gas in the central region to be below $T_{\text {vir }}$, it is easier to heat the gas (to $\sim T_{\text {vir }}$ ) in the central region than to permanently separate it from the dark matter potential well (i.e. the BCG). Simulations suggest that cluster mergers can destroy cooling flows (e.g. Burns et al. 2008, and references therein). It is not clear though that such heating need be done by something as energetic as a major merger. For instance, A1650 has an almost flat temperature profile and yet no evidence for a recent major merger (Donahue et al. 2005). Other examples of such clusters are WCC clusters A2244 (Donahue et al. 2005), A1651 and A1060.

To explore this further we compared the central stellar velocity dispersion of the BCG to the central temperature. The BCG central stellar velocity dispersions were collected from HyperLeda, using mean reported values and standard deviation of the values for the uncertainty. In a few cases, if only one measurement was reported, we used the reported error on the measurement. 16 of our 64 HIFLUGCS clusters (including 8 SCC clusters) did not have any data available. Figure 18 shows BCG central stellar velocity dispersion versus $k T_{0}$. The points 


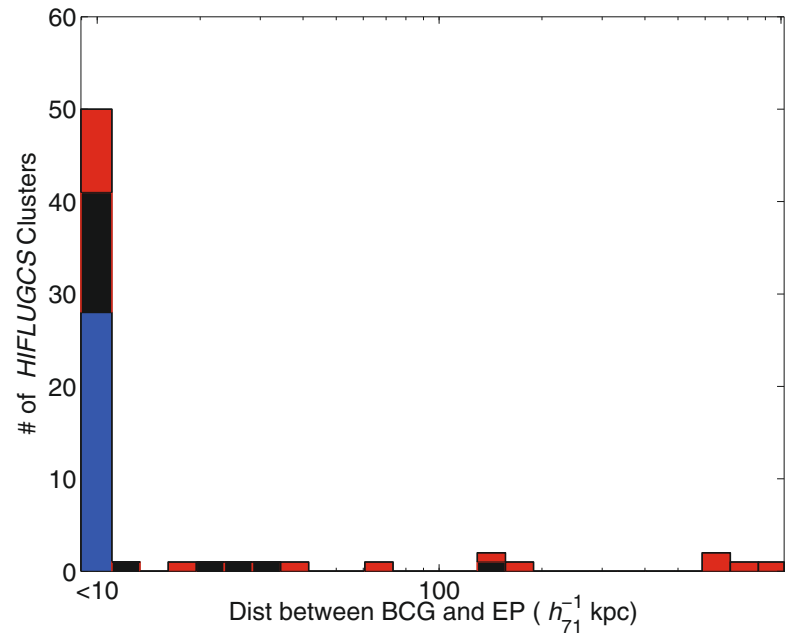

Fig. 17. Histogram of projected separation of the BCG and X-ray emission peak. The colors represent: blue - SCC clusters, black - WCC clusters and red - NCC clusters. Most of the clusters ( 78\%) have a BCG within $12 h_{71}^{-1} \mathrm{kpc}$ of the X-ray peak. $100 \%$ of the SCC clusters, $61 \%$ of the WCC clusters and $50 \%$ of the NCC clusters have a BCG within $12 h_{71}^{-1} \mathrm{kpc}$ of the X-ray peak. The uncertainty in the X-ray peaks is generally $4^{\prime \prime}$, which corresponds to $\sim 4 h_{71}^{-1} \mathrm{kpc}$ for our median redshift.

are color coded: SCC-blue, WCC-black and NCC-red. Clusters with a central temperature decline are marked with a circle and clusters without a central temperature drop are marked with a square. The 16 clusters with no data available are omitted, but have the same general distribution in $k T_{0}$ as the 48 plotted clusters. Even when looking at just the SCC clusters, there seems to be only a weak correlation between the BCG's central velocity dispersion and $k T_{0}$.

Assuming that our above conjecture is correct, that the gas is relatively easy to heat without removing it from the center of the potential, then it appears that the temperature of the central gas is not generally determined by purely gravitational processes in the local potential. That is, some heating mechanism heats the gas either slightly above the local virial temperature in SCC clusters or up to $\sim T_{\text {vir }}$ in WCC and NCC clusters. We emphasize that the mechanisms do not have to be the same in all cases (e.g. possible AGN outbursts or sloshing for SCC and WCC borderline cases and mergers for NCC clusters). This would explain the large scatter in Fig. 18. It would also predict that the central gas in clusters such as A1650 will eventually return to the local virial temperature in the center. In such a case, in order to maintain pressure equilibrium with the surrounding gas, the density would increase dramatically, shortening the CCT and returning the cluster to the SCC subsample. It is not clear in this model which clusters would be capable of returning to their SCC status and which ones not. Perhaps the early major mergers of NCC clusters, as proposed by Burns et al. (2008), prevent the formation of the large central galaxy needed to reform the CC. Perhaps once the entropy is increased enough, the gas cannot cool to the local virial temperature in the time available. Even when suppressed by magnetic fields, thermal conduction is still likely to increase rapidly with temperature. Given that its effect is already argued to be significant (e.g. Zakamska \& Narayan 2003), it may well play a role in preventing cool cores from being re-established after disruption by mergers in hot clusters. We suggest that simulations of the formation of CCs considering different central potentials could help understand which ones can

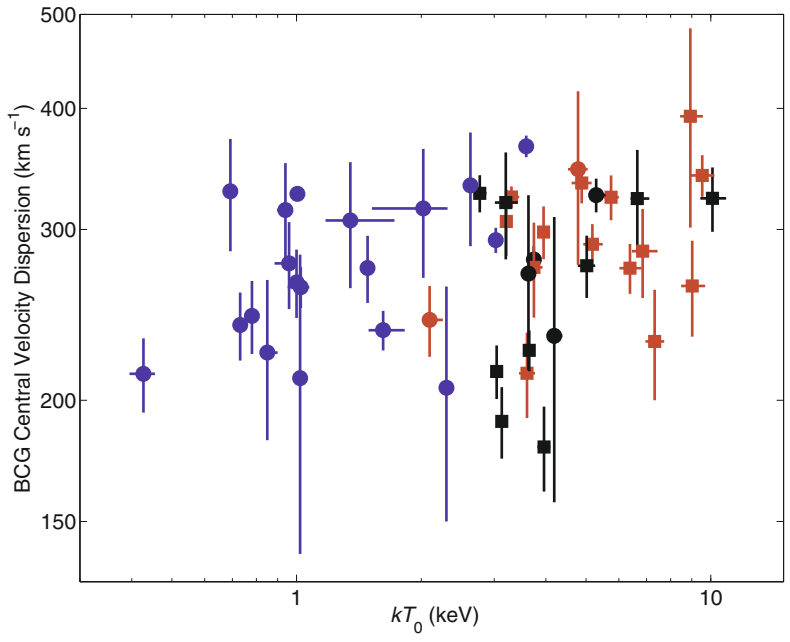

Fig. 18. BCG's central velocity dispersion versus $k T_{0}$. Blue points are SCC clusters, black points are WCC clusters and red points are NCC clusters. Circles signify clusters with a central temperature drop and squares signify clusters without a central temperature drop. The 16 clusters with no BCG velocity dispersion data available span the same range. There appears to be no correlation between the BCG's central velocity dispersion and $k T_{0}$.

re-form and which cannot and what physical effects are important in determining the fate of the central gas.

\subsection{BCG separation and mergers}

Several works show a special relationship between the cooling activity in cluster cores and the brightest cluster galaxies located within a certain projected distance from the X-ray peak, typically $50 h_{71}^{-1} \mathrm{kpc}$, to the X-ray peak (see Mittal et al. 2009 , for more details). Given that only eight of our 64 clusters have a significant $\left(>50 h_{71}^{-1} \mathrm{kpc}\right)$ projected separation between the X-ray peak and BCG suggests that separating the BCG from the gas is more difficult than simply heating the gas and/or disrupting the cooling flow. Looking more deeply, the eight clusters with BCG-X-ray peak separations $>50 h_{71}^{-1} \mathrm{kpc}$ ordered from largest to smallest are: (1) A3376 - 939 $h_{71}^{-1} \mathrm{kpc}$, (2) $\mathrm{A} 0754-\sim 714 h_{71}^{-1} \mathrm{kpc}$, (3) A1367 - 666 $h_{71}^{-1} \mathrm{kpc}$, (4) A1736 - $\sim 642 h_{71}^{-1} \mathrm{kpc}$, (5) A3667 - 155 $h_{71}^{-1} \mathrm{kpc}$, (6) A2163 $\sim 128 h_{71}^{-1} \mathrm{kpc}$, (7) A2256 - 110 $h_{71}^{-1} \mathrm{kpc}$ and (8) A2255 $\sim 72 h_{71}^{-1} \mathrm{kpc}^{21}$. All eight of these clusters have been identified as merging clusters, A3667 is the only CC cluster (and it is a borderline CC/NCC cluster) and all except A1736 have been identified as having a radio halo and/or relic(s) (see Appendix $\mathrm{C}$ for individual references). The appearance of such diffuse, Mpc scale non-thermal emission is thought to be powered by major mergers. Of the other 56 clusters, only eight (15\%) have been identified as possibly containing diffuse non-thermal emission on large scales: A1656, A3562, A85, A133, A401, A2152, A4038 and MKW8. Of these eight, for two (A401 and A2142) the detections seem unlikely (Giovannini \& Feretti 2000), three (A85, A133 and A4038) are small scale (a few tens of kpc) relics associated with nearby radio galaxies (e.g. Slee et al. 2001) and one (MKW8) as identified as a possible relic as seen in the VLA Low-Frequency Sky Survey (VLSS) at $74 \mathrm{MHz}$ (Cohen et al. 2007). Kempner et al. (2004) identified three classes of

\footnotetext{
21 Additionally the peculiar velocity of A2255's BCG is much larger than that of any other cluster (greater than A2255's velocity dispersion).
} 
radio relics that were fundamentally different. We argue that in these four cases, the relics are not the large scale Gischt associated with mergers, but $A G N$-relics. This leaves only two (4\%) unambiguous detections of large scale diffuse radio emission: the Coma cluster and A3562. Based on the large discrepancy between the number of clusters with large scale $(\sim \mathrm{Mpc})$ radio structure that have a large BCG-EP separation and those that do not, we argue that a large separation of the EP and BCG is a very good indication a major merger and therefore could be applied as a useful method for discovering radio halos and radio relics-Gischt.

\subsection{WCC clusters}

The WCC clusters are an interesting set because they seem to occupy a transition between NCC and SCC clusters. They are defined as having short to moderate CCTs (1.0-7.7 $h_{71}^{-1 / 2} \mathrm{Gyr}$ ), and generally have flat or shallow central temperature drops and a central entropy that is enhanced compared to their SCC cousins. Due to their flat or shallow temperature profile, they are mostly classified as NCC clusters in studies that determine CC clusters by a central temperature drop. Recently Burns et al. (2008) suggested that $\mathrm{CC}$ clusters were not necessarily more relaxed than NCC clusters. Since Burns et al. (2008) define CC/NCC classification based on temperature drop, they would classify the WCC clusters as NCC clusters. Could these WCC clusters be the relaxed NCCs? Donahue et al. (2005) studied two radio quiet, relaxed WCC clusters - A1650 and A2244. They conclude that these objects are CC clusters that have had a major AGN outburst, raising the central entropy and temporarily disrupting the $\mathrm{CF}$.

In an effort to distinguish between these two possibilities we examined the X-ray morphology of the WCC and NCC clusters. We divided these 36 clusters into four types: (I) relaxed with short CCT $\left(<3 h_{71}^{-1 / 2} \mathrm{Gyr}\right.$ ), (II) relaxed with moderate/long CCT ( $>3 h_{71}^{-1 / 2} \mathrm{Gyr}$ ), (III) disturbed with short CCT $\left(<3 h_{71}^{-1 / 2} \mathrm{Gyr}\right)$, (IV) disturbed with moderate/long CCT ( $\left.>3 h_{71}^{-1 / 2} \mathrm{Gyr}\right)$. We classify relaxed versus disturbed by visual inspection. We define a relaxed cluster as having: (1) round or elliptical isophotes; (2) the EP at the center of the isophotes and (3) little or no offset between the centers of the different levels of isophotes (little or no sloshing). We note, based on our classification scheme, that all our NCC clusters will be of type II or IV. Our physical interpretation of the four types are: (I) CCs that have been disrupted, but will re-form - their CCTs are too short to be relaxed NCCs, (II) clusters that have not had a recent merger but have had their CC severely disrupted (or destroyed) by a previous major merger (III) clusters that are merging with the core surviving (although it may be destroyed in the future) and (IV) merging clusters in which there was no cool core or the core has been destroyed.

In order to check our categorization based on visual inspection, we plotted the distance between the EP and the X-ray emission weighted center (EWC) (see Hudson et al. 2006, for details on determining the EWC). This method gives a simple way to classify clusters, since disturbed clusters will generally have a larger distance between their EP and EWC than a relaxed clusters. Figure 19 shows the results for the $36 \mathrm{WCC}$ and NCC clusters. Black points indicate the clusters identified as being relaxed and the red points indicate the disturbed clusters. Figure 19 confirms that, in general, the disturbed clusters have a larger separation between the EP and EWC than the relaxed clusters.

We find seven clusters of type I, six clusters of type II, five clusters of type III and 18 clusters of type IV. By the definition

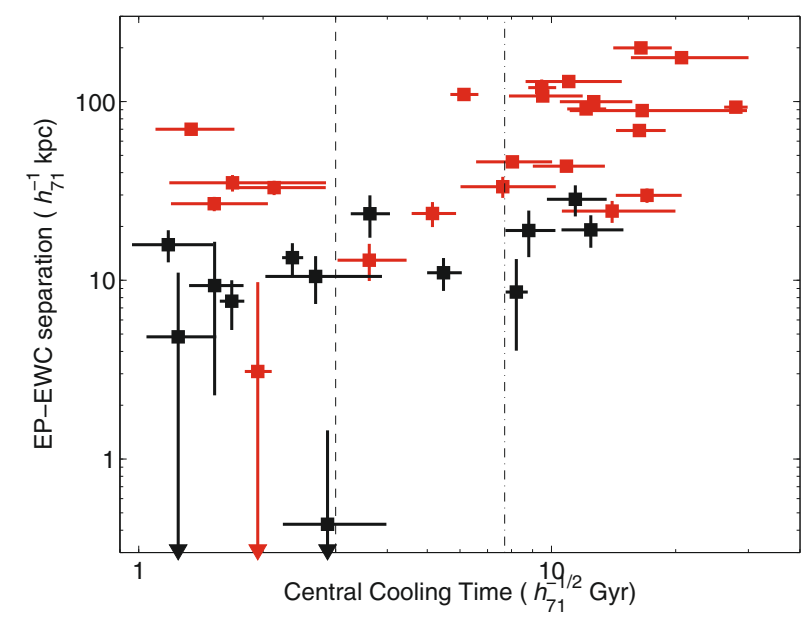

Fig. 19. Distance between the X-ray emission peak and emission weighted center for the $36 \mathrm{WCC}$ and NCC clusters. The black points are clusters that appear relaxed and the red points are clusters that appear disturbed. The vertical dashed line separates clusters with short CCT and long CCT. The dash-dot line divides the clusters between WCC and NCC. It is clear that in general the separation of the EP and EWC is larger for the apparently disturbed clusters. The error on the separation is $4^{\prime \prime}$, which is the typical size of the smoothing kernel used when determining the EP.

of Burns et al. (2008), these 36 clusters are clusters that will never form a CC. We argue, however, given the short cooling time of the clusters of type I, it is unlikely that their CCT will never drop below $1 h_{71}^{-1 / 2} \mathrm{Gyr}$, which in turn implies they will also have a central temperature drop. $39 \%$ of the WCC clusters are of this type and are perhaps a special type of SCC cluster that experienced an anomalous event that has temporarily disrupted the strong cool core (e.g. raised the central temperature, entropy and cooing time).

We acknowledge that a more sophisticated method should be employed when determining whether a cluster is relaxed or not. However based on preliminary results, unless WCC clusters (especially those with short CCT) are fundamentally different from SCC clusters, some of them will become SCC clusters. That is, unless there is a process that keeps their CCT $>1 h_{71}^{-1 / 2} \mathrm{Gyr}$ and their temperature-profile flat, the gas in the core will cool below $T_{\text {vir }}$ and the CCT will drop below unity.

\section{Conclusions}

We provide the most detailed systematic view into X-ray cores of galaxy clusters to date. We find that the best method to determine whether a cluster is a cool-core (CC) cluster is with the central cooling time (CCT). We divide clusters into three types: strong cool-core (SCC), weak cool-core (WCC or transition) and non-cool-core clusters. SCC are defined as having very short CCT $\left(<1 h_{71}^{-1 / 2} \mathrm{Gyr}\right)$ and are characterized by low central entropy $\left(\$ 30 h_{71}^{-1 / 3} \mathrm{keV} \mathrm{cm}^{2}\right)$, systematic central temperature drops (with $T_{0} / T_{\text {vir }} \sim 0.4$ ) and a brightest cluster galaxy (BCG) at the X-ray peak. WCC or transition clusters are defined as having moderate CCT ( CCT between $1-7.7 h_{71}^{-1 / 2} \mathrm{Gyr}$ ) and are characterized as having an elevated entropy ( $\left.\gtrsim 30 h_{71}^{-1 / 3} \mathrm{keV} \mathrm{cm}^{2}\right)$, flat or slightly decreasing central temperature profiles and having a BCG at or near $\left(<50 h_{71}^{-1} \mathrm{kpc}\right)$ the X-ray peak. NCC clusters are defined as having long CCT ( $\left.>7.7 h_{71}^{-1 / 2} \mathrm{Gyr}\right)$, and are characterized by 
large central entropies $\left(K_{0}>110 h_{71}^{-1 / 3} \mathrm{keV} \mathrm{cm}^{2}\right)$ and temperature profiles that generally are flat or rise towards the center.

Based on the above classification, our main conclusions are:

1. In our flux-limited statistically complete sample, we find $72 \%$ of the clusters are CC clusters with $44 \%$ of the clusters being SCC clusters and $28 \%$ being WCC clusters.

2. For intermediate redshift clusters (where radii as small as $0.4 \% r_{500}$ cannot be resolved), we find that $K_{\mathrm{BIAS}}$ and scaled $L_{\mathrm{X}}$ are the best proxies for CCT. In general $K_{\mathrm{BIAS}}$ is better, but needs to be standardized before it is used. We suggest using $I(R) \equiv 2 \pi \int_{-\infty}^{\infty} \int_{0}^{R} n_{\mathrm{e}} n_{\mathrm{H}} r \mathrm{~d} r \mathrm{~d} l$ with a possible value of $I=8.6 \times 10^{65} h_{71}^{-2} \mathrm{~cm}^{-3}$

3. For high redshift clusters (clusters with very few counts), we find that cuspiness is the most useful proxy. However, it suffers from a large scatter in the relation to CCT, so precise categorizations can be difficult. Preliminary results suggest that a concentration parameter $(\Sigma(<40 \mathrm{kpc}) / \Sigma(<400 \mathrm{kpc}))$, as suggested by Santos et al. (2008), may serve as the best proxy for distant clusters.

4. Dividing our representative sample into four redshift bins, we find it unlikely that the discrepancy between spectral mass deposition rate $\left(\dot{M}_{\text {spec }}\right)$ and classical mass deposition rate $\left(\dot{M}_{\text {classical }}\right)$ is due to the fact that CCs formed very recently. The probability that the lowest- and the highestredshift clusters with non-zero forward-evolved mass deposition rates come from the same population is extremely low $(<3 \%)$. This requires the discrepancy to be explained by some heating method.

5. There is no evidence found in our work for a universal central temperature profile as claimed previously for smaller samples. This suggests that the radius of the cool gas is not universal. As seen in Table 2, the radius within which the temperature falls below $T_{\text {vir }}$ differs from cluster to cluster.

6. We find the majority ( 78\%) of HIFLUGCS clusters, including $100 \%$ of the SCC clusters, $61 \%$ of the WCC clusters and $50 \%$ of the NCC clusters, have a BCG within $12 h_{71}^{-1} \mathrm{kpc}$ of the X-ray emission peak. This number increases to $88 \%$ for a distance of $50 h_{71}^{-1} \mathrm{kpc}$. We find that seven out of eight clusters with the BCG-EP separation $>50 h_{71}^{-1} \mathrm{kpc}$ have Mpc scale radio emission (halo or relic-Gischt) versus only two of the remaining 56 with a BCG very close to the EP.

7. There is a weak correlation between the SCC central temperature and the cooling of the gas as predicted by the cooling flow model. We also find no correlation between the SCC central temperature and the central velocity dispersion of the BCG. We interpret this, along with the result that so many clusters have a BCG at their peak, as indicating that the central temperature of the gas is influenced by heating, which occurs differently in different clusters. Therefore, the central temperature is not simply reflective of the central potential well or the expected cooling rate from $T_{\text {high }}$ to $T_{\text {low }}$, but requires more complex physics.

8. We find that $<39 \%$ of the WCC clusters which are relaxed also have relatively short cooling times $\left(t_{\text {cool }}<3 h_{71}^{-1 / 2} \mathrm{Gyr}\right)$. We argue that these clusters are similar to SCC clusters but have had an event that temporarily disrupted the cool core, raising the core temperature, entropy and cooling time.

Results obtained here on cluster cool-core properties and their correlations, using a complete well-controlled sample with simple selection criteria, can be taken as benchmark for next generation cosmological hydrodynamical simulations aiming at describing detailed properties of cluster cores.
Acknowledgements. The authors wish to thank D. A. Buote, T. E. Clarke, M. Markevitch and A. Vikhlinin for providing proprietary data before it was publicly available. We would like to thank E. Murphy for providing pointed radio measurements of $N_{\mathrm{H}}$ for several clusters. We thank E. Blanton, H. Böhringer, Y. Ikebe, E. Pierpaoli, S. Randall, P. Schuecker, and G. Sivakoff for help in the early stages of this work. We would like to thank R. Smith for providing the data for the APEC model. We thank the referee for a beneficial feedback and James Wicker for useful correspondence. T. H. R. and D. S. H. acknowledge support from the Deutsche Forschungsgemeinschaft through Emmy Noether research grant RE 1462/2. R. M. acknowledges support from the Deutsche Forschungsgemeinschaft through the Schwerpunkt Program 1177 (RE 1462/4). H. A. acknowledges financial support from CONACyT under grants 50921-F and 81356, and partial support from the Transregional Collaborative Research Center TRR33 "The Dark Universe". P. E. J. N. acknowledges support from NASA grant NAS8-03060. C. L. S. was supported in part by NASA Chandra grants GO7-8129X and AR7-8012X and NASA XMM-Newton grant NNX06AE76G. This research has made use of the NASA/IPAC Extragalactic Database (NED) which is operated by the Jet Propulsion Laboratory, California Institute of Technology, under contract with the National Aeronautics and Space Administration. We acknowledge the usage of the HyperLeda database (http://leda. univ-lyon 1.fr).

\section{References}

Akritas, M. G., \& Bershady, M. A. 1996, ApJ, 470, 706

Allen, S. W., Fabian, A. C., Johnstone, R. M., Arnaud, K. A., \& Nulsen, P. E. J. 2001a, MNRAS, 322, 589

Allen, S. W., Schmidt, R. W., \& Fabian, A. C. 2001b, MNRAS, 328, L37

Andernach, H., Tago, E., Einasto, M., Einasto, J., \& Jaaniste, J. 2005, in Nearby Large-Scale Structures and the Zone of Avoidance, ed. A. P. Fairall, \& P. A. Woudt, ASP Conf. Ser., 329, 283

Ashman, K. M., Bird, C. M., \& Zepf, S. E. 1994, AJ, 108, 2348

Bagchi, J., Durret, F., Neto, G. B. L., \& Paul, S. 2006, Science, 314, 791

Bauer, F. E., Fabian, A. C., Sanders, J. S., Allen, S. W., \& Johnstone, R. M. 2005, MNRAS, 359, 1481

Baumgartner, W. H., \& Mushotzky, R. F. 2006, ApJ, 639, 929

Belsole, E., Birkinshaw, M., \& Worrall, D. M. 2005, MNRAS, 358, 120

Bîrzan, L., McNamara, B. R., Nulsen, P. E. J., Carilli, C. L., \& Wise, M. W. 2008, ApJ, 686, 859

Bîrzan, L., Rafferty, D. A., McNamara, B. R., Wise, M. W., \& Nulsen, P. E. J. 2004, ApJ, 607, 800

Blanton, E. L., Sarazin, C. L., McNamara, B. R., \& Wise, M. W. 2001, ApJ, 558, L15

Böhringer, H., Burwitz, V., Zhang, Y.-Y., Schuecker, P., \& Nowak, N. 2005, ApJ, 633,148

Bonamente, M., Nevalainen, J., \& Lieu, R. 2007, ApJ, 668, 796

Bridle, A. H., \& Fomalont, E. B. 1976, A\&A, 52, 107

Buote, D. A., \& Lewis, A. D. 2004, ApJ, 604, 116

Burns, J. O., Hallman, E. J., Gantner, B., Motl, P. M., \& Norman, M. L. 2008, ApJ, 675, 1125

Cavagnolo, K. W., Donahue, M., Voit, G. M., \& Sun, M. 2009, ApJS, 182, 12

Cavaliere, A., \& Fusco-Femiano, R. 1976, A\&A, 49, 137

Chatzikos, M., Sarazin, C. L., \& Kempner, J. C. 2006, ApJ, 643, 751

Chen, Y., Reiprich, T. H., Böhringer, H., Ikebe, Y., \& Zhang, Y.-Y. 2007, A\&A, 466,805

Clarke, T. E., \& Ensslin, T. A. 2006, AJ, 131, 2900

Clarke, T. E., Blanton, E. L., \& Sarazin, C. L. 2004, ApJ, 616, 178

Cohen, A. S., Lane, W. M., Cotton, W. D., et al. 2007, AJ, 134, 1245

Condon, J. J., Cotton, W. D., Greisen, E. W., et al. 1998, AJ, 115, 1693

Cortese, L., Gavazzi, G., Boselli, A., Iglesias-Paramo, J., \& Carrasco, L. 2004, A\&A, 425, 429

Cortese, L., Gavazzi, G., Boselli, A., et al. 2006, A\&A, 453, 847

Cowie, L. L., \& Binney, J. 1977, ApJ, 215, 723

Crawford, C. S., Sanders, J. S., \& Fabian, A. C. 2005, MNRAS, 361, 17

De Grandi, S., \& Molendi, S. 2002, ApJ, 567, 163

de Plaa, J., Kaastra, J. S., Tamura, T., et al. 2004, A\&A, 423, 49

Dennis, T. J., \& Chandran, B. D. G. 2005, ApJ, 622, 205

Donahue, M. 2007, in Heating versus Cooling in Galaxies and Clusters of Galaxies, ESO Astrophysics Symposia, ed. H. Böhringer, G. W. Pratt, A. Finoguenov, \& P. Schuecker (Heidelberg, Berlin: Springer-Verlag), 20

Donahue, M., Voit, G. M., O’Dea, C. P., Baum, S. A., \& Sparks, W. B. 2005, ApJ, 630, L13

Donnelly, R. H., Forman, W., Jones, C., et al. 2001, ApJ, 562, 254

Drinkwater, M. J., Gregg, M. D., \& Colless, M. 2001, ApJ, 548, L139

Dunn, R. J. H., \& Fabian, A. C. 2006, MNRAS, 373, 959

Dunn, R. J. H., \& Fabian, A. C. 2008, ArXiv e-prints, 801

Dupke, R., White, III, R. E., \& Bregman, J. N. 2007a, ApJ, 671, 181 
Dupke, R. A., Mirabal, N., Bregman, J. N., \& Evrard, A. E. 2007b, ApJ, 668, 781

\section{Edge, A. C. 2001, MNRAS, 328, 762}

Evrard, A. E., Metzler, C. A., \& Navarro, J. F. 1996, ApJ, 469, 494

Fabian, A. C., \& Nulsen, P. E. J. 1977, MNRAS, 180, 479

Fabian, A. C., \& Nulsen, P. E. J. 1979, MNRAS, 186, 783

Fabian, A. C., Sanders, J. S., Ettori, S., et al. 2001, MNRAS, 321, L33

Feretti, L., Böhringer, H., Giovannini, G., \& Neumann, D. 1997, A\&A, 317, 432

Feretti, L., Dallacasa, D., Govoni, F., et al. 1999, A\&A, 344, 472

Feretti, L., \& Giovannini, G. 1994, A\&A, 281, 375

Feretti, L., Fusco-Femiano, R., Giovannini, G., \& Govoni, F. 2001, A\&A, 373, 106

Finoguenov, A., Henriksen, M. J., Briel, U. G., de Plaa, J., \& Kaastra, J. S. 2004, ApJ, 611, 811

Finoguenov, A., Henriksen, M. J., Miniati, F., Briel, U. G., \& Jones, C. 2006, ApJ, 643, 790

Finoguenov, A., Reiprich, T. H., \& Böhringer, H. 2001, A\&A, 368, 749

Fujita, Y., Sarazin, C. L., Kempner, J. C., et al. 2002, ApJ, 575, 764

Fujita, Y., Sarazin, C. L., Reiprich, T. H., et al. 2004, ApJ, 616, 157

Gavazzi, G., \& Trinchieri, G. 1983, ApJ, 270, 410

Giacintucci, S., Venturi, T., Brunetti, G., et al. 2005, A\&A, 440, 867

Giovannini, G., \& Feretti, L. 2000, New Astron., 5, 335

Giovannini, G., Tordi, M., \& Feretti, L. 1999, New Astron., 4, 141

Gonzalez, A. H., Zabludoff, A. I., Zaritsky, D., \& Dalcanton, J. J. 2000, ApJ, 536,561

Guo, F., \& Oh, S. P. 2008, MNRAS, 384, 251

Henriksen, M. J., \& Tittley, E. R. 2002, ApJ, 577, 701

Henriksen, M. J., \& White, III, R. E. 1996, ApJ, 465, 515

Henry, J. P., Finoguenov, A., \& Briel, U. G. 2004, ApJ, 615, 181

Hudson, D. S., \& Reiprich, T. H. 2007, in Heating versus Cooling in Galaxies and Clusters of Galaxies, ed. H. Böhringer, G. W. Pratt, A. Finoguenov, \& P. Schuecker, 42

Hudson, D. S., Reiprich, T. H., Clarke, T. E., \& Sarazin, C. L. 2006, A\&A, 453, 433

Ikebe, Y., Reiprich, T. H., Böhringer, H., Tanaka, Y., \& Kitayama, T. 2002, A\&A, 383,773

Johnstone, R. M., Fabian, A. C., Morris, R. G., \& Taylor, G. B. 2005, MNRAS, 356,237

Kaastra, J. S., Ferrigno, C., Tamura, T., et al. 2001, A\&A, 365, L99

Kalberla, P. M. W., Burton, W. B., Hartmann, D., et al. 2005, A\&A, 440, 775

Kassim, N. E., Clarke, T. E., Enßlin, T. A., Cohen, A. S., \& Neumann, D. M. 2001, ApJ, 559, 785

Kempner, J. C., Blanton, E. L., Clarke, T. E., et al. 2004, in The Riddle of Cooling Flows in Galaxies and Clusters of galaxies, ed. T. Reiprich, J. Kempner, \& N. Soker, 335

Kempner, J. C., \& David, L. P. 2004, ApJ, 607, 220

Kempner, J. C., Sarazin, C. L., \& Ricker, P. M. 2002, ApJ, 579, 236

Kuntz, K. D. 2001, Ph.D. Thesis, NASA Goddard Space Flight Center

Lea, S. M., Silk, J., Kellogg, E., \& Murray, S. 1973, ApJ, 184, L105

Łokas, E. L., Wojtak, R., Gottlöber, S., Mamon, G. A., \& Prada, F. 2006, MNRAS, 367, 1463

Machalski, J., \& Condon, J. J. 1999, ApJS, 123, 41

Makishima, K., Ezawa, H., Fukuzawa, Y., et al. 2001, PASJ, 53, 401

Mann, H. B., \& Whitney, D. R. 1947, Annals of Mathematical Statistics, 18, 50

Markevitch, M., \& Vikhlinin, A. 2001, ApJ, 563, 95

Markevitch, M., Forman, W. R., Sarazin, C. L., \& Vikhlinin, A. 1998, ApJ, 503, 77

Markevitch, M., Ponman, T. J., Nulsen, P. E. J., et al. 2000, ApJ, 541, 542

Markevitch, M., Vikhlinin, A., \& Mazzotta, P. 2001, ApJ, 562, L153

Mathews, W. G., \& Bregman, J. N. 1978, ApJ, 224, 308

Mathews, W. G., Faltenbacher, A., \& Brighenti, F. 2006, ApJ, 638, 659

Mazzotta, P., Brunetti, G., Giacintucci, S., Venturi, T., \& Bardelli, S. 2004, J. Korean Astron. Soc., 37, 381

McNamara, B. R., \& O’Connell, R. W. 1989, AJ, 98, 2018

McNamara, B. R., Wise, M. W., Nulsen, P. E. J., et al. 2001, ApJ, 562, L149

Mewe, R., Gronenschild, E. H. B. M., \& van den Oord, G. H. J. 1985, A\&AS, 62, 197

Mewe, R., Lemen, J. R., \& van den Oord, G. H. J. 1986, A\&AS, 65, 511

Mittal, R., Hudson, D. S., Reiprich, T. H., \& Clarke, T. 2009, A\&A, 501, 835

Molendi, S., \& Pizzolato, F. 2001, ApJ, 560, 194
Morris, R. G., \& Fabian, A. C. 2005, MNRAS, 358, 585

Mushotzky, R. F., \& Szymkowiak, A. E. 1988, in NATO ASIC Proc. 229: Cooling Flows in Clusters and Galaxies, ed. A. C. Fabian, 53

Nevalainen, J., Oosterbroek, T., Bonamente, M., \& Colafrancesco, S. 2004, ApJ, 608, 166

Nulsen, P. E. J., McNamara, B. R., Wise, M. W., \& David, L. P. 2005, ApJ, 628, 629

Oegerle, W. R., Cowie, L., Davidsen, A., et al. 2001, ApJ, 560, 187

O’Hara, T. B., Mohr, J. J., Bialek, J. J., \& Evrard, A. E. 2006, ApJ, 639, 64

Paturel, G., Petit, C., Prugniel, P., et al. 2003, A\&A, 412, 45

Peterson, J. R., Kahn, S. M., Paerels, F. B. S., et al. 2003, ApJ, 590, 207

Peterson, J. R., Paerels, F. B. S., Kaastra, J. S., et al. 2001, A\&A, 365, L104

Rafferty, D. A., McNamara, B. R., Nulsen, P. E. J., \& Wise, M. W. 2006, ApJ, 652,216

Reiprich, T. H. 2006, A\&A, 453, L39

Reiprich, T. H., \& Böhringer, H. 2002, ApJ, 567, 716

Reiprich, T. H., \& Hudson, D. S. 2007, in Heating versus Cooling in Galaxies and Clusters of Galaxies, ed. H. Böhringer, G. W. Pratt, A. Finoguenov, \& P. Schuecker, 381

Reiprich, T. H., Hudson, D. S., Zhang, Y. ., et al. 2009, A\&A, 501, 899

Reiprich, T. H., Sarazin, C. L., Kempner, J. C., \& Tittley, E. 2004, ApJ, 608, 179 Roettiger, K., Burns, J. O., \& Stone, J. M. 1999, ApJ, 518, 603

Rossetti, M., Ghizzardi, S., Molendi, S., \& Finoguenov, A. 2007, A\&A, 463, 839

Ruszkowski, M., Brüggen, M., \& Begelman, M. C. 2004, ApJ, 611, 158

Sakelliou, I., Peterson, J. R., Tamura, T., et al. 2002, A\&A, 391, 903

Sakelliou, I., \& Ponman, T. J. 2004, MNRAS, 351, 1439

Sanders, J. S., \& Fabian, A. C. 2002, MNRAS, 331, 273

Sanders, J. S., Fabian, A. C., Allen, S. W., et al. 2008, MNRAS, 385, 1186

Sanderson, A. J. R., Finoguenov, A., \& Mohr, J. J. 2005, ApJ, 630, 191

Sanderson, A. J. R., Ponman, T. J., \& O’Sullivan, E. 2006a, MNRAS, 372, 1496

Sanderson, A. J. R., Ponman, T. J., \& O'Sullivan, E. 2006b, MNRAS, 372, 1496

Santos, J. S., Rosati, P., Tozzi, P., et al. 2008, A\&A, 483, 35

Sato, K., Yamasaki, N. Y., Ishida, M., et al. 2007, PASJ, 59, 299

Slee, O. B., \& Roy, A. L. 1998, MNRAS, 297, L86

Slee, O. B., Roy, A. L., Murgia, M., Andernach, H., \& Ehle, M. 2001, AJ, 122, 1172

Smith, R. K., \& Brickhouse, N. S. 2000, in ed. S. J. Arthur, N. S. Brickhouse, \& J. Franco, Rev. Mex. Astron. Astrof. Conf. Ser., 9, 134

Smith, R. K., Brickhouse, N. S., Liedahl, D. A., \& Raymond, J. C. 2001, in Spectroscopic Challenges of Photoionized Plasmas, ed. G. Ferland, \& D. W. Savin, ASP Conf. Ser., 247, 161

Sun, M., \& Vikhlinin, A. 2005, ApJ, 621, 718

Sun, M., Murray, S. S., Markevitch, M., \& Vikhlinin, A. 2002, ApJ, 565, 867

Sun, M., Vikhlinin, A., Forman, W., Jones, C., \& Murray, S. S. 2005, ApJ, 619, 169

Takizawa, M., Sarazin, C. L., Blanton, E. L., \& Taylor, G. B. 2003, ApJ, 595, 142

Tamura, T., Bleeker, J. A. M., Kaastra, J. S., Ferrigno, C., \& Molendi, S. 2001a, A\&A, 379, 107

Tamura, T., Kaastra, J. S., Peterson, J. R., et al. 2001b, A\&A, 365, L87

Tittley, E. R., \& Henriksen, M. 2001, ApJ, 563, 673

Venturi, T., Bardelli, S., Morganti, R., \& Hunstead, R. W. 2000, MNRAS, 314, 594

Vikhlinin, A., Burenin, R., Forman, W. R., et al. 2007, in Heating versus Cooling in Galaxies and Clusters of Galaxies, ed. H. Böhringer, G. W. Pratt, A. Finoguenov, \& P. Schuecker, 48

Vikhlinin, A., Markevitch, M., \& Murray, S. S. 2001a, ApJ, 551, 160

Vikhlinin, A., Markevitch, M., \& Murray, S. S. 2001b, ApJ, 549, L47

Vikhlinin, A., Markevitch, M., Murray, S. S., et al. 2005, ApJ, 628, 655

Vikhlinin, A., Burenin, R. A., Ebeling, H., et al. 2009, ApJ, 692, 1033

Voit, G. M., Cavagnolo, K. W., Donahue, M., et al. 2008, ApJ, 681, L5

Werner, N., Kaastra, J. S., Takei, Y., et al. 2007, A\&A, 468, 849

Wilcoxon, F. 1945, Biometrics, 1, 80

Willson, M. A. G. 1970, MNRAS, 151, 1

Xu, H., Kahn, S. M., Peterson, J. R., et al. 2002, ApJ, 579, 600

Yamasaki, N. Y., Ohashi, T., \& Furusho, T. 2002, ApJ, 578, 833

Zakamska, N. L., \& Narayan, R. 2003, ApJ, 582, 162

Zappacosta, L., Buote, D. A., Gastaldello, F., et al. 2006, ApJ, 650, 777

Pages 27 to 40 are available in the electronic edition of the journal at http: //www . aanda. org 


\section{Appendix A: Calculating central density for a double $\beta$ model}

Starting from the definition of the normalization of the APEC model (Mewe et al. 1985, 1986; Smith \& Brickhouse 2000; Smith et al. 2001) and taking $n(r)=n_{\mathrm{e}}$ with $\zeta=\frac{n_{\mathrm{e}}}{n_{\mathrm{H}}}$, (calculated individually, but generally $\sim 1.2$ ),

$\mathcal{N} \equiv \frac{10^{-14}}{4 \pi D_{\mathrm{A}} D_{\mathrm{L}} \zeta} \int n(r)^{2} \mathrm{~d} V$

where the terms are defined as in Eq. (9). For a double $\beta$ model the expression for $n(r)$ is:

$n(r)=\left[n_{01}^{2}\left(1+\left(\frac{r}{r_{\mathrm{c}_{1}}}\right)^{2}\right)^{-3 \beta_{1}}+n_{02}^{2}\left(1+\left(\frac{r}{r_{\mathrm{c}_{2}}}\right)^{2}\right)^{-3 \beta_{2}}\right]^{\frac{1}{2}}$.

The unabsorbed ${ }^{22}$ surface brightness at a projected distance, $x$ from the center over an energy range between $E_{1}$ and $E_{2}$ is

$\Sigma(x)=\frac{\int_{E_{1}}^{E_{2}} \Lambda_{\mathrm{X}}(T, Z, E) \mathrm{d} E}{4 \pi(1+z)^{4} \zeta} \int_{-\infty}^{\infty} n(r)^{2} \mathrm{~d} l$,

where $r^{2}=x^{2}+l^{2}$ and $\Lambda_{\mathrm{X}}(T, Z, E)$ is the emissivity function for a plasma of temperature $T$ and metalicity $Z$ at energy $E$. This can be rewritten in terms of $n_{01}$ and $n_{02}$ as:

$\Sigma(x)=\frac{\int_{E_{1}}^{E_{2}} \Lambda_{\mathrm{X}}(T, Z, E) \mathrm{d} E}{4 \pi(1+z)^{4} \zeta} \int_{-\infty}^{\infty} n_{01}^{2}\left(1+\left(\frac{x^{2}+l^{2}}{x_{\mathrm{c}_{1}}^{2}}\right)\right)^{-3 \beta_{1}} \mathrm{~d} l+\frac{\int_{E_{1}}^{E_{2}} \Lambda_{\mathrm{X}}(T, Z, E) \mathrm{d} E}{4 \pi(1+z)^{4} \zeta} \int_{-\infty}^{\infty} n_{02}^{2}\left(1+\left(\frac{x^{2}+l^{2}}{x_{\mathrm{c}_{2}}^{2}}\right)\right)^{-3 \beta_{2}} \mathrm{~d} l$.

Solving the integral gives the standard expression for the double $\beta$ model in terms of surface brightness:

$\Sigma(x)=\Sigma_{01}\left(1+\left(\frac{x}{x_{\mathrm{c}_{1}}}\right)^{2}\right)^{-3 \beta_{1}+\frac{1}{2}}+\Sigma_{02}\left(1+\left(\frac{x}{x_{\mathrm{c}_{2}}}\right)^{2}\right)^{-3 \beta_{2}+\frac{1}{2}}$,

where

$\Sigma_{0 i} \equiv \frac{n_{0 i}^{2} \int_{E_{1}}^{E_{2}} \Lambda_{\mathrm{X}}(T, Z, E) \mathrm{d} E}{4 \pi(1+z)^{4} \zeta} \int_{-\infty}^{\infty}\left(1+\left(\frac{l}{x_{\mathrm{c}_{\mathrm{i}}}}\right)^{2}\right)^{-3 \beta_{i}} \mathrm{~d} l=\frac{n_{0 i}^{2} x_{\mathrm{c}_{\mathrm{i}}} \pi^{\frac{1}{2}}}{4 \pi(1+z)^{4} \zeta} \frac{\Gamma\left(3 \beta_{i}-\frac{1}{2}\right)}{\Gamma\left(3 \beta_{i}\right)} \int_{E_{1}}^{E_{2}} \Lambda_{\mathrm{X}}(T, Z, E) \mathrm{d} E$.

Therefore,

$\frac{n_{01}^{2}}{n_{02}^{2}}=\frac{\Sigma_{01} \mathrm{LI}_{2}}{\Sigma_{02} \mathrm{LI}_{1}}=\frac{\Sigma_{12} \mathrm{LI}_{2}}{\mathrm{LI}_{1}}$

where $\mathrm{LI}_{i}$ and $\Sigma_{12}{ }^{23}$ are as defined in Eq. (12). Using this relation along with the fact that $n_{0} \equiv n(0)=\sqrt{n_{01}^{2}+n_{02}^{2}}$, we find:

$n_{01}^{2}=\frac{\Sigma_{12} \mathrm{LI}_{2}}{\Sigma_{12} \mathrm{LI}_{2}+\mathrm{LI}_{1}} n_{0}^{2}$

and

$n_{02}^{2}=\frac{\mathrm{LI}_{1}}{\Sigma_{12} \mathrm{LI}_{2}+\mathrm{LI}_{1}} n_{0}^{2}$

Inserting these values into Eq. (A.2) to find an expression for $n(r)$ in terms of $n_{0}$, we get

$n(r)=\frac{n_{0}}{\sqrt{\Sigma_{12} \mathrm{LI}_{2}+\mathrm{LI}_{1}}}\left[\Sigma_{12} \mathrm{LI}_{2}\left(1+\left(\frac{r}{r_{\mathrm{c}_{1}}}\right)^{2}\right)^{-3 \beta_{1}}+\mathrm{LI}_{1}\left(1+\left(\frac{r}{r_{\mathrm{c}_{2}}}\right)^{2}\right)^{-3 \beta_{2}}\right]^{\frac{1}{2}}$.

Inserting this expression of $n(r)$ into Eq. (A.1) and solving for $n_{0}$, we recover Eq. (12).

${ }^{22}$ In our case Eq. (A.1) already takes into account the absorption and any absorption in the subsequent calculations cancels.

${ }^{23}$ Note, we have explicitly assumed $\Lambda_{\mathrm{X}}(T, Z, E)$ is the same for both components. In the case it is not, $\Sigma_{12}$ can be redefined as $\Lambda_{\mathrm{X}}\left(T_{2}, Z_{2}, E\right) \Sigma_{01} / \Lambda_{\mathrm{X}}\left(T_{1}, Z_{1}, E\right) \Sigma_{02}$ and the calculations follow identically. 


\section{Appendix B: $K_{\mathrm{BIAS}}$ calculations}

From the definition of surface brightness (Eq. (A.3)), a cluster at redshift $z$, of a region with an angular radius $x$, has an integrated surface brightness (or Flux $\mathcal{F}$ ) between energies $E_{1}$ and $E_{2}$ :

$\mathcal{F}=\frac{\int_{E_{1}}^{E_{2}} \Lambda_{\mathrm{X}}(T, Z, E) \mathrm{d} E}{2(1+z)^{4}} \int_{-\infty}^{\infty} \int_{0}^{x} n_{\mathrm{e}} n_{\mathrm{H}} x \mathrm{~d} x \mathrm{~d} l$,

where $n_{\mathrm{e}}$ is the electron density, $n_{\mathrm{H}}$ is the proton density, $\Lambda_{\mathrm{X}}(T, Z, E)$ is the emissivity function as defined in Eq. (A.3). To remove the redshift dependence of the projected region size, we convert the projected region of angular radius $x$ to a cylindrical region of physical radius $R$, such that $R \approx x D_{\mathrm{A}}(z)$. Equation (B.1) becomes:

$\mathcal{F}=\frac{\int_{E_{1}}^{E_{2}} \Lambda_{\mathrm{X}}(T, Z, E) \mathrm{d} E}{4 \pi D_{\mathrm{A}} D_{\mathrm{L}}(1+z)^{2}} I(R)$,

where $D_{\mathrm{A}}$ is angular diameter distance, $D_{\mathrm{L}}$ is the luminosity distance and $I(R)$ is defined as in Eq. (20). Therefore the total counts $C$ collected by a telescope for an observation of length $t_{\mathrm{obs}}$, in an energy band from $E_{1}$ to $E_{2}$, of a cylindrical region of physical radius $R$ is:

$C=t_{\mathrm{obs}} \frac{\int_{E_{1}}^{E_{2}} \alpha(E) \Lambda_{\mathrm{X}}(T, Z, E) A_{\mathrm{eff}}(E) \mathrm{d} E}{4 \pi D_{\mathrm{A}} D_{\mathrm{L}}(1+z)^{2}} I(R)$,

where $\alpha(E)$ and $A_{\text {eff }}(E)$ are the absorption from Galactic hydrogen and the effective area of the telescope at energy $E$, respectively. We can calculate $\int_{E_{1}}^{E_{2}} \alpha(E) \Lambda_{\mathrm{X}}(T, Z, E) A_{\mathrm{eff}}(E) \mathrm{d} E$ for an absorbed thermal model using XSPEC with an appropriate ARF and RMF. Specifically, since normalization $\mathcal{N} \propto C R \equiv C / t_{\mathrm{obs}}, X S P E C$ can be used to find the constant of proportionality $\kappa$. From the definition of $\mathcal{N}$ (see Eq. (A.1)):

$\int_{E_{1}}^{E_{2}} \alpha(E) \Lambda_{\mathrm{X}}(T, Z, E) A_{\mathrm{eff}}(E) \mathrm{d} E=\frac{(1+z)^{2} \kappa}{10^{14}}$,

so that

$C=t_{\mathrm{obs}} \frac{10^{-14} \kappa}{4 \pi D_{\mathrm{A}} D_{\mathrm{L}}} I(R)$.

Using an on-axis Chandra ARF and RMF, we determined $\kappa\left(122.3\right.$ photons $\left.\mathrm{cm}^{5} \mathrm{~s}^{-1}\right)$ for an energy band from $0.5-7.0 \mathrm{keV}$, with $Z=0.25$ solar, $N_{\mathrm{H}}=2 \times 10^{20} \mathrm{~cm}^{-2}$ and our median observation time (44 ks), redshift $(0.047)$ and virial temperature (4.3 keV). Inserting our determined value of $\kappa$ into Eq. (B.5) and solving for $I$, such that $C=10000$ counts, yields $I=8.6 \times 10^{65} h_{71}^{-2} \mathrm{~cm}^{-3}$. Therefore using the criterion that our median observation would have $K_{\text {BIAS }}$ determined by circle with 10000 counts, equivalent regions from other observations would have:

$\frac{C}{\mathrm{cnts}}=17910\left(\frac{t_{\mathrm{obs}}}{100 \mathrm{ks}}\right)\left(\frac{\kappa}{100 \mathrm{cnts} \mathrm{cm}^{5} \mathrm{~s}^{-1}}\right)\left(\frac{200 h_{71}^{-1} \mathrm{Mpc}}{D_{\mathrm{A}}(1+z)}\right)^{2}$.

\section{Appendix C: Notes on individual clusters}

\section{C.1. $A 0085$}

This cluster appears to have two subclumps, one near the center and one further to the south (Kempner et al. 2002). In determining the temperature profile and global cluster temperature the latter was excluded. This SCC cluster hosts a well-studied radio relic, which is close to but not connected to the central radio galaxy (e.g. Slee et al. 2001). The central region of this cluster requires a double thermal model out to $\sim 11^{\prime \prime}\left(\sim 12 h_{71}^{-1} \mathrm{kpc}\right)$.

\section{C.2. $A 0119$}

This is possibly a merging cluster, which shows elongation towards the northeast. The X-ray peak of this NCC cluster, which does not dominate the surface brightness, has a cD galaxy cospatial with it. The cluster contains three wide-angle-tailed (WAT) radio galaxies which may be interacting with the ICM (e.g. Feretti et al. 1999).

\section{C.3. $A 0133$}

Central regions of this cluster show an east-west elongation. An in-depth study with XMM and Chandra by Fujita et al. (2004, 2002) revealed an X-ray tongue extending northwest. This SCC cluster hosts a radio relic, that is close to but not connected to the central radio galaxy (e.g. Slee et al. 2001). The central region of this cluster requires a double thermal model out to $\sim 15^{\prime \prime}\left(\sim 16 h_{71}^{-1} \mathrm{kpc}\right)$. 


\section{C.4. NGC 0507 group}

The overall X-ray spectrum of this group shows a suspicious hard tail. An additional powerlaw component was included in the overall temperature fit. It is possible that the hard tail is due to unresolved low mass X-ray binaries (LMXBs), although the central region $\left(\sim 1 \prime 8=35.7 h_{71}^{-1} \mathrm{kpc}\right)$ was removed and no evidence of a hard excess is seen in the spectra of the central annuli. It is possible that (given the redshift of NGC $0507 z=0.0165$ ) the LMXBs are only strong enough to be measured in a large region and are insignificant compared to the group emission in the central region. On the other hand the powerlaw has a steep photon index $\left(\Gamma_{X}=2.3-3.0\right)$ that is not consistent with LMXBs, which usually have a photon index of $\Gamma_{X}=1.6$. The component has a total flux of $\sim 4 \times 10^{-12} \mathrm{erg} \mathrm{cm}^{-2} \mathrm{~s}^{-1}$ corresponding to a luminosity of $2 \times 10^{42} h_{71}^{-2} \mathrm{erg} \mathrm{s}^{-1}$ (over 0.4-10.0 keV). It is also possible that the hard excess is related to an insufficiently subtracted particle background, which is visible in this cool cluster. Both models (additional particle background or powerlaw) give an identical overall temperature. We also note that a more detailed analysis of the residual background in outer cluster regions shows no residual particle background. The central region of this cluster requires a double thermal model out to $\sim 62^{\prime \prime}\left(\sim 20.6 h_{71}^{-1} \mathrm{kpc}\right)$.

\section{C.5. $A 0262$}

The spectral fits to the inner regions are poor $\left(\chi^{2} /\right.$ d.o.f. $\left.~ 1.4\right)$ even with a double thermal model. Using non-solar abundance ratios significantly improves the fit, but does not change the best-fit overall temperature. We therefore used solar ratios for simplicity. The central region of this cluster requires a double thermal model out to $\sim 43^{\prime \prime}\left(\sim 14 h_{71}^{-1} \mathrm{kpc}\right)$.

\section{C.6. $A 0400$}

This cluster hosts the double radio source 3C75 within its center and shows evidence of merging (Hudson et al. 2006). As noted in Hudson et al. (2006), the hydrogen column density is higher than measured in the radio $\left(N_{\mathrm{H}}=0.85 \times 10^{21} \mathrm{~cm}^{-2}\right.$ Kalberla et al. 2005 ) and therefore we left it free for all spectral fits. We find a hydrogen column density of $N_{\mathrm{H}}=0.98-1.22 \times 10^{21} \mathrm{~cm}^{-2}$ for our fit to the overall cluster.

\section{C.7. A0399}

This cluster is near to A401 and shows evidence of interaction with A401 (e.g. Sakelliou \& Ponman 2004). The temperature profile of this cluster peaks at the X-ray center.

\section{C.8. $A 0401$}

See also A0399. This cluster may host a radio halo (Giovannini et al. 1999). We included an early observation (before 2001), since the later observation was offset, with the cluster center in the corner of a CCD. The BCG closest to the X-ray peak is $\sim 34 h_{71}^{-1} \mathrm{kpc}$ away, making it one of fourteen clusters with the BCG $>12 h_{71}^{-1} \mathrm{kpc}$ from the X-ray peak.

\section{C.9. $A 3112$}

Although the background flaring seen in some observations was removed, the effect seems to have broadened a fluorescence line. This can be seen in the fit to the overall cluster spectrum (at $\sim 7.5 \mathrm{keV}$ ). This effect seems simply to make the fit poor ( $\chi^{2} /$ d.o.f. $~ 1.6$ ), but it does not affect the best-fit values whether the line is removed or not. Takizawa et al. (2003) first presented the Chandra data of A3112, interpreting the radio active central cD galaxy as interacting with the ICM. Bonamente et al. (2007) claim a soft excess and hard excess in this cluster that may be related with the central radio active BCG. We do not see a similar effect, however we do not separately fit the $1^{\prime}-2 \cdot 5$ annulus that Bonamente et al. (2007) fit. We do confirm that the $1^{\prime}-2.5$ annulus is isothermal in our $k T$-profile so that their result is not due to a temperature fluctuations in the cluster. This SCC cluster is one of sixteen clusters for which no data exist for the BCG central velocity dispersion.

\section{C.10. NGC 1399/Fornax cluster}

This nearby SCC cluster has two X-ray peaks cospatial with NGC 1399 and NGC 1404. The X-ray peak is taken to be cospatial with the BCG NGC 1399. The peak on NGC 1404 was removed for spatial and spectral analysis. Fornax appears to be an outlier in six of the plots of parameters versus CCT in which it has an anomalously low value for its CCT. These parameters are: (1) $\Sigma_{0}$; (2) $r_{\mathrm{c}} / r_{500}$; (3) $K_{\text {BIAS }}$; (4) cooling radius; (5) $\dot{M}_{\text {classical }} / M_{500}$ and (6) $M_{\text {gas }} / M_{500}$. Additionally it is the only SCC cluster in which $\dot{M}_{\text {classical }} \leq \dot{M}_{\text {spec }}$. One possible explanation is that $N G C 1404$ is about $0.04 r_{500}$ from the X-ray peak and due to the extended emission around it, the surface brightness profile severely flattens. The extrapolated outer profile therefore overestimates the projected gas lowering the central density (and altering associated parameters). To check how much this influenced the Fornax cluster as an outlier, we fit only the central part of the surface brightness that could be fit well to a single $\beta$-model. This model most likely underestimates the projected gas, thereby providing the largest possible values for central density. In the case of Fig. 10, this model raises $\dot{M}_{\text {classical }}$ to $0.75 \pm 0.04 h_{71}^{-2} M_{\odot} \mathrm{yr}^{-1}$ making it larger than $\dot{M}_{\text {spectral }}=0.52 \pm 0.02 M_{\odot} \mathrm{yr}^{-1}$. We emphasize that this result overestimates $\dot{M}_{\text {classical }}$ and in any case $\dot{M}_{\text {spec }} \approx \dot{M}_{\text {classical }}$, making it an odd SCC cluster. In all other cases the Fornax cluster remains an outlier. In the case of the CCT the value falls to $\sim 0.4 h_{71}^{-1 / 2}$ Gyr moving the Fornax cluster to the left in Fig. 6. (1) $\Sigma_{0}$, not surprisingly, remains 
almost the same, $\sim 0.21 \times 10^{-6}$ photons $\mathrm{cm}^{-2} \mathrm{~s}^{-1} \mathrm{arcsec}^{-2}$. The shift to the left in this case makes the Fornax cluster even more of an outlier. (2) $r_{\mathrm{c}} / r_{500}$, likewise, remains almost identical, $\sim 4 \times 10^{-4}$. Unlike, $\Sigma_{0}$, the shift to the left in this case makes the Fornax cluster more similar to other SCC clusters. (3) $K_{\text {BIAS }}$ is, of course, unaffected by the density model, however moving the Fornax cluster to the left makes it more consistent with the other SCC clusters. (4) The cooling radius remains almost identical, increasing to $0.03 r_{500}$. Additionally moving the Fornax cluster to the left makes it even more of an outlier. (5) $\dot{M}_{\text {classical }} / M_{500}$ increases slightly to $\sim 1.8 \times 10^{-14} h_{71}^{-1} \mathrm{yr}^{-1}$. Moving the Fornax cluster to the left makes the trend of decreasing $\dot{M}_{\text {classical }} / M_{500}$ with CCT worse, but it is more consistent with other groups with short CCT. (6) $M_{\text {gas }} / M_{500}$, is raised slightly, $\sim 0.2 \times 10^{-3}$. Similar to $\dot{M}_{\text {classical }} / M_{500}$, moving the Fornax cluster to the left makes the trend with CCT worse, but makes it more consistent with the other groups. We emphasize that the Fornax cluster is still an outlier in all six cases, and that these values are the other extrema, with the true value somewhere between these and where they are plotted in Fig. 6. The physical interpretation is that it is possible that Fornax is in the process of forming a cool core (i.e. it has a nascent core in the terms of Burns et al. (2008)) and therefore is dynamically different from the other SCC clusters that have well-established cores. The central region of this cluster requires a double thermal model out to $\sim 170^{\prime \prime}$ $\left(\sim 15.9 h_{71}^{-1} \mathrm{kpc}\right)$.

\section{C.11. $2 A 0335+096$}

This cluster, along with A0478 and NGC 1550, has a significantly higher hydrogen column density than measured at radio wavelengths $\left(N_{\mathrm{H}}=1.68 \times 10^{21} \mathrm{~cm}^{-2}\right.$ Kalberla et al. 2005). We fit all spectra with the column density free. For the fit to the overall spectrum we find $N_{\mathrm{H}}=2.35-2.47 \times 10^{21} \mathrm{~cm}^{-2}$. This cluster has two major galaxies near the X-ray peak, which resides between the two of them $\left(\sim 10 h_{71}^{-1} \mathrm{kpc}\right.$ from the closest). Of the 16 clusters in which no information about the BCG central velocity dispersion is available, this cluster has the shortest CCT. The central region of this cluster requires a double thermal model out to $\sim 38^{\prime \prime}$ $\left(\sim 26 h_{71}^{-1} \mathrm{kpc}\right)$.

\section{C.12. IIIZw54}

$I I I Z w 54$ is a pair of galaxies near the center of a poor galaxy group. We used a 6 " kernel when smoothing the image in order to determine the emission peak. This cluster does not have a bright core, although it appears to be quite round and relaxed. The brighter of two galaxies in the galaxy pair IIIZw54 (a cD galaxy) is cospatial with the X-ray peak.

\section{C.13. $A 3158$}

Łokas et al. (2006) report A3158 as a relaxed cluster based on the velocity dispersion of the galaxies. The X-ray emission appears to be elliptical and there are two cDs near the cluster center, one of which lies at the X-ray peak. This cluster definitely does not have a bright core, with a central density of only $\sim 5 \times 10^{-3} \mathrm{~cm}^{-3}$. The temperature profile peaks in the center at $\sim 5.7 \mathrm{keV}$.

\section{C.14. $A 0478$}

This cluster, along with 2A0335+096 and NGC 1550, has a significantly higher hydrogen column density than measured in the radio $\left(N_{\mathrm{H}}=1.64 \times 10^{21} \mathrm{~cm}^{-2}\right.$ Kalberla et al. 2005). We fit all spectra with the column density free. Our fit to the overall cluster yields a column density of $\left(N_{\mathrm{H}}=2.89-2.96 \times 10^{21} \mathrm{~cm}^{-2}\right)$, consistent with the value found by Sanderson et al. (2005). This cluster has the highest spectral mass deposition rate of any HIFLUGCS cluster, making it an ideal candidate for a grating observation. Unfortunately the RGS data from a long XMM-Newton exposure was virtually unusable (de Plaa et al. 2004). This SCC cluster is also one of sixteen clusters in which no data for the BCG central velocity dispersion are available.

\section{C.15. NGC 1550 group}

This cluster, along with 2A0335+096 and A0478, has a significantly higher hydrogen column density than measured in the radio $\left(N_{\mathrm{H}}=0.981 \times 10^{21} \mathrm{~cm}^{-2}\right.$ Kalberla et al. 2005). We fit all spectra with the column density free. Our fit to the overall cluster yields a column density of $N_{\mathrm{H}}=1.34-1.41 \times 10^{21} \mathrm{~cm}^{-2}$. The column density appears to peak towards the center of the cluster, having a value of $N_{\mathrm{H}}=1.9-2.2 \times 10^{21} \mathrm{~cm}^{-2}$ in the innermost annulus.

\section{C.16. EXO0422-086/RBS 0540}

The short observation of this SCC cluster indicates a round, centrally peaked cluster with a moderate central temperature drop. The BL Lac object EXO 0423.4-0840 at the center of this cluster was studied by Belsole et al. (2005). This is one of sixteen clusters for which no data about the BCG central velocity dispersion are available.

\section{C.17. A3266}

This cluster has a very low background scaling factor; therefore an additional unfolded powerlaw component was included in the spectral fits to account for any residual particle background. Reading in the background as a corfile (i.e. a second background component with an adjustable scaling factor), the overall best-fit temperature is found to be consistent with our result including an unfolded powerlaw. Henriksen \& Tittley (2002); Finoguenov et al. (2006) presented detailed analyses of this merging system. 


\section{C.18. $A 0496$}

The high abundances in the central region of this cluster are better fit with a VAPEC model, however since this did not change the best-fit values of temperature, solar ratios were used for simplicity. A double thermal model greatly improved the fits to spectra in annuli out to 0.3. However, the high temperature component for annuli between 0.18 and 0.3 is $\gg k T_{\text {vir }}(k T \sim 8 \mathrm{keV})$. Although this may be evidence of very hot gas near the cluster core, the investigation is beyond the scope of this paper. Therefore for annuli between 0.18 and 0.3, we used a single thermal fit. Dupke et al. (2007a) studied the longest of the three Chandra observations of this cluster in depth. They argue that there is a cold front at the center of this cluster, which is caused by an off-center passage of a smaller dark matter halo.

\section{C.19. $A 3376$}

This cluster was fit with an unfolded powerlaw component to account for possible low-level flares in both observations. This cluster appears highly disturbed in the X-ray with a strong elongation along the east-west direction. Bagchi et al. (2006) report the existence of double relics, one to the east of the cluster center and one to the west. Nevalainen et al. (2004) found a diffuse, hard excess with the BeppoSAX PDS at $2.7 \sigma$ significance. The BCG of this cluster is $\sim 1 \mathrm{Mpc}$ from the X-ray peak, the most distant of any cluster in the sample and one of eight clusters with a separation of $>50 h_{71}^{-1} \mathrm{kpc}$. There is a radio galaxy with bent jets very close to the X-ray peak (Mittal et al. 2009). Optically it is clearly much fainter than the BCG and is most likely an AGN that may have been activated by the merger. The jets are bent in the opposite direction to the elongation of the cluster, possibly bent from ICM ram pressure.

\section{C.20. $A 3391$}

The short observation of this NCC cluster shows an elliptical shaped ICM with a BCG cospatial with the emission peak. Tittley \& Henriksen (2001) discovered a filament between A3391 and the nearby cluster A3395.

\section{C.21. A3395s}

This cluster is very close to and may be interacting with A3395e. A3395e was excluded from all extended analysis. Donnelly et al. (2001) claim A3395s and A3395e are near first core passage.

\section{C.22. $A 0576$}

Kempner \& David (2004) originally presented an analysis of the Chandra data. Dupke et al. (2007b) presented a detailed analysis of the XMM-Newton and Chandra data suggesting that it is a line-of-sight merger. The X-ray image seems somewhat perturbed with elliptical isophotes with alternating NW-SE shifted centers, reminiscent of sloshing, already noted by Kempner \& David (2004). The BCG is $\sim 24 h_{71}^{-1} \mathrm{kpc}$ from the X-ray peak, making it one of fourteen clusters with the separation $>12 h_{71}^{-1} \mathrm{kpc}$. There is, however, a slightly fainter galaxy closer $\left(<12 h_{71}^{-1} \mathrm{kpc}\right)$ to the X-ray peak that is radio active, whereas the BCG is not. The peculiar velocity of the BCG is one of five clusters that is more than $50 \%$ of the velocity dispersion. This WCC cluster is one of the three WCC/NCC clusters with CCT $\gg 1 h_{71}^{-1 / 2}$ Gyr (i.e. not on the border between SCC and WCC) and a systematic temperature decrease at the cluster center.

\section{C.23. $A 0754$}

This irregularly shaped cluster hosts a halo and relic (Kassim et al. 2001). Henry et al. (2004) presented a detailed analysis of this merging system using the XMM-Newton observation. Only the pre-2001 Chandra observation is used, since it was the only one that contained the cluster core. More recent observation have been made but do not cover the cluster center and therefore are not useful for core studies. The BCG for this cluster is $\sim 714 h_{71}^{-1} \mathrm{kpc}$ away from the X-ray peak, making it one of eight clusters where this separation is $>50 h_{71}^{-1} \mathrm{kpc}$.

\section{C.24. A0780/Hydra-A cluster}

This cluster is known to have a massive central AGN outburst (Nulsen et al. 2005).

\section{C.25. A1060}

This WCC cluster is also known as the Hydra cluster. Sato et al. (2007) recently presented a Suzaku observation of this cluster. This cluster has two bright galaxies near the core, one of which is cospatial with X-ray peak. Both galaxies have a clearly visible diffuse X-ray component (Yamasaki et al. 2002).

\section{C.26. $A 1367$}

Due to the short exposure time and lack of a bright core, we used a $12^{\prime \prime}$ kernel when smoothing to determine the X-ray peak. This is a very well-studied merging cluster. This cluster has an infalling starburst group (Sun et al. 2005; Cortese et al. 2006) and 
optical evidence suggests that this is a merging system (Cortese et al. 2004). The X-ray image appears rather disturbed with several off-centered bright sources. Sun \& Vikhlinin (2005) studied the survival of galaxy coronae in this system. This cluster hosts a radio relic (Gavazzi \& Trinchieri 1983). The BCG of this cluster is $\sim 666 h_{71}^{-1} \mathrm{kpc}$ from the X-ray peak making it one of eight clusters where this separation is $>50 h_{71}^{-1} \mathrm{kpc}$. It is also one of five clusters where the BCG peculiar velocity is $>50 \%$ of the cluster velocity dispersion.

\section{C.27. MKW4}

A single thermal model is a poor fit to this high metalicity center. Although a second thermal model does provide an improvement, freeing the ratio of elements for a single thermal model provides the best-fit. Since freeing the abundance ratios does not change the overall best-fit temperatures of the annuli, solar ratios with a single thermal model were used for simplicity.

\section{C.28. $Z w C l 1215.1+0400$}

The short observation of this NCC cluster, shows a round cluster with no bright central peak and an elliptical BCG located at the $\mathrm{X}$-ray emission peak. This is one of the sixteen clusters for which no data about the BCG central velocity dispersion are available. The BCG of this clusters is also $\sim 18 h_{71}^{-1} \mathrm{kpc}$ from the X-ray peak, making it one of fourteen clusters where this separation is $>12 h_{71}^{-1} \mathrm{kpc}$.

\section{C.29. NGC 4636 group}

This nearby group contains extended nonthermal emission in the central region extending out $\sim 122^{\prime \prime}\left(\sim 9.19 h_{71}^{-1} \mathrm{kpc}\right)$. The luminosity of this emission $\left(L_{\mathrm{X}} \sim 10^{40} h_{71}^{-2} \mathrm{erg} \mathrm{s}^{-1}\right)$ is consistent with the expected unresolved LMXB population for NGC 4636. In addition to a powerlaw component, the central region of this cluster requires a double thermal model out to $\sim 35^{\prime \prime}\left(\sim 2.6 h_{71}^{-1} \mathrm{kpc}\right)$.

\section{C.30. A3526/Centaurus cluster}

This is a well-studied, prototypical CC cluster, with a central temperature drop (having the largest fractional drop, $k T_{0} \sim 0.2 k T_{\text {vir }}$ ) and enhanced central metalicity. An arc-like X-ray feature near the center has been identified as most likely being a cold front associated with sloshing in the core (Sanders \& Fabian 2002). The central region of this cluster requires a double thermal model out to $\sim 72^{\prime \prime}\left(\sim 16.5 h_{71}^{-1} \mathrm{kpc}\right)$.

\section{C.31. $A 1644$}

As with A0085, this SCC cluster shows evidence of merging, with the existence of a double X-ray peak. Reiprich et al. (2004) analyzed the XMM EPIC observation of this cluster. They found the flux of the northern (smaller) subclump is below the HIFLUGCS flux limit whereas the flux of the southern (larger) subclump is above the flux limit. Therefore for purposes of this analysis the smaller subclump was excluded from spatial and spectral analysis. Additionally Reiprich et al. (2004) found evidence that the smaller subclump was being stripped as it passes through the ICM. This is one of sixteen clusters in which the central velocity dispersion of the BCG is unavailable. The central region of this cluster requires a double thermal model out to $\sim 32^{\prime \prime}\left(\sim 30 h_{71}^{-1} \mathrm{kpc}\right)$.

\section{C.32. $A 1650$}

This CC cluster hosts a radio quiet cD galaxy (Donahue et al. 2005). Mittal et al. (2009) find an upper-limit to the bolometric radio luminosity of $\sim 9 \times 10^{38} h_{71}^{-2} \mathrm{erg} \mathrm{s}^{-1}$. The original short Chandra observation showed a flat temperature profile (Donahue et al. 2005). However, the longer, mosaiced observations show a slight temperature decrease in the central region. Due to the elevated entropy in the core, Donahue et al. (2005) concluded a major AGN outburst had disrupted the cooling flow. This cluster is one of four clusters on the border between SCC and WCC. Its CCT $\left(\sim 1.2 h_{71}^{-1 / 2} \mathrm{Gyr}\right)$ is slightly longer than the 1 Gyr cutoff. Moreover this cluster shows a central temperature decrease typical of SCC clusters. This is one of sixteen clusters in which the central velocity dispersion of the BCG is unavailable.

\section{C.33. A1651}

As with A1650, Donahue et al. (2005) claim this is a radio quiet CC cluster, however Mittal et al. (2009) detect central radio emission with a bolometric luminosity of $\sim 10^{40} h_{71}^{-2} \mathrm{erg} \mathrm{s}^{-1}$. Gonzalez et al. (2000) fit the optical light out to $\sim 670 h_{100}^{-1} \mathrm{kpc}$, over one quarter of $r_{\text {vir }}$. The X-ray structure looks quite round and shows no evidence of external interaction. However, the X-ray peak does not dominate as much as in SCC clusters and there is no evidence of a central temperature drop. This WCC is one of sixteen clusters in which the central velocity dispersion of the BCG is unavailable. 


\section{C.34. A1656/Coma cluster}

This well-studied NCC cluster appears to be involved in a merger with a group. This cluster hosts the first detected radio halo (Willson 1970).

\section{C.35. NGC 5044 troup}

The spectra for the inner regions of this group are not well fit by a single thermal model $\left(\chi^{2} /\right.$ d.o.f. $\left.>2\right)$. After trying several different models to fit the residuals, we found that the statically best model which is also physically motivated is a thermal model that allows oxygen, silicon, sulfur, and iron to vary from solar ratios and a powerlaw to account for a the clear hard tail (most likely due to LMXBs). We note that a double thermal model with the above elements not constrained at solar ratios provides the statistically best-fit (in the innermost annulus $\chi^{2} /$ d.o.f. $=1.04$ vs. $\chi^{2} /$ d.o.f. $=1.13$ for the thermal plus powerlaw model). In this model, however, the hotter thermal model has a temperature of $k T=1.4-3.0$ which is hotter than any gas found in the outer annuli and the measured virial temperature $\left(k T_{\text {vir }}=1.22_{-0.04}^{+0.03}\right)$. Unless there is a hot halo of gas extending from the center of the group out to $\sim 16 h_{71}^{-1} \mathrm{kpc}$, this model is unphysical. Finally, we note that adding a powerlaw to the second thermal model does not improve the fit and similar high temperatures are found for the hotter thermal component as for the simple two thermal model.

\section{C.36. $A 1736$}

This NCC cluster is a member of the Shapley Supercluster. Due to the short exposure time and lack of a bright core, the X-ray peak was found by smoothing the image with a $\sim 10^{\prime \prime}$ kernel. The X-ray morphology shows an irregular shape with no well-defined core. A preliminary temperature map shows heating to the east and west of the emission peak with cool gas extending to the south. The BCG of this cluster is $\sim 642 h_{71}^{-1} \mathrm{kpc}$ from the peak, making it one of eight clusters where this separation is $>50 h_{71}^{-1} \mathrm{kpc}$. This is the only cluster with a separation of $>50 h_{71}^{-1} \mathrm{kpc}$ that does not have any known associated radio halo or relic.

\section{C.37. $A 3558$}

This WCC cluster is located in the core of the Shapley Supercluster. The observation was heavily flared, and even after a conservative cleaning of the light curve there was evidence of some low-level flaring in the back-illuminated chips. Rossetti et al. (2007) presented the $X M M$ and Chandra analysis of this cluster, concluding that it had a cool core that had survived a merger. We find evidence of a slight temperature drop in the core of this WCC cluster.

\section{C.38. $A 3562$}

This WCC cluster is located in the core of the Shapley Supercluster. The X-ray emission from this cluster appears to be elongated along the northeast-southwest direction. Giacintucci et al. (2005) report the detection of a radio halo (also see Venturi et al. 2000) and argue for a merger scenario between A3562 and SC 1329-313. Finoguenov et al. (2004) presented a detailed analysis of the $X M M$ observation of this cluster. The BCG of this cluster is $\sim 31 h_{71}^{-1} \mathrm{kpc}$ from the X-ray peak, making it one of fourteen clusters where this separation is $>12 h_{71}^{-1} \mathrm{kpc}$. However, the BCG is located in a chip gap, so the separation may simply be an instrumental effect (i.e. the peak on the BCG may not be detected due to the chip gap). The XMM observation also shows an offset between the $\mathrm{X}$-ray peak and BCG but on a scale of only $\sim 23 h_{71}^{-1} \mathrm{kpc}$ (Zhang, private communication).

\section{C.39. $A 3571$}

This WCC cluster is a member of the Shapley Supercluster.

\section{C.40. $A 1795$}

The core of this well-studied SCC cluster has a large filament seen in X-rays and H $\alpha$ (Crawford et al. 2005). Early core studies with Chandra were done by Fabian et al. (2001) and Markevitch et al. (2001). Fabian et al. (2001) found a CCT of $\sim 0.4 h_{71}^{-1 / 2}$ Gyr, approximately the same age as they estimate for the filament. The difference between their measurement for CCT and our measurement is probably due to the different values of $r_{\text {cool }}$ used to determine CCT. In order to keep consistency between clusters we determined the CCT at $0.004 r_{500}$, however at the redshift of A1795 we are able to determine the CCT at an even smaller radius which gives a CCT $\sim 0.5 h_{71}^{-1 / 2} \mathrm{Gyr}$, consistent with Fabian et al. (2001). Moreover, their technique for determining CCT is slightly different from ours. Markevitch et al. (2001) found a cold front in the core of A1795, which they attribute to sloshing gas. Oegerle et al. (2001) studied FUSE observations and found an upper limit for $\dot{M}_{\text {spec }}(20 \mathrm{kpc})<28 h_{71}^{-1 / 2} M_{\odot} \mathrm{yr}^{-1}$, consistent with our measurement of $\sim 15 M_{\odot} \mathrm{yr}^{-1}$.

\section{C.41. $A 3581$}

This SCC cluster is a member of the Hydra-Centaurus Supercluster. The central region of this cluster requires a double thermal model out to $\sim 40^{\prime \prime}\left(\sim 18 h_{71}^{-1} \mathrm{kpc}\right)$. Johnstone et al. (2005) analyzed the Chandra data from A3581. They find a point source 
coincident with the powerful radio source PKS 1404-267 at the cluster center. They find a central temperature drop to $\sim 0.4 k T_{\text {vir }}$ at the cluster center, similar to our measurement of $\sim 0.5 k T_{\text {vir }}$.

\section{C.42. MKW8}

This NCC cluster shows little substructure in the X-ray image. The X-ray isophotes are elliptical with the major axis along the east-west direction. The isophotes seem to have a common center (i.e. no evidence of sloshing), however the X-ray peak appears to lie to the east of the center of the isophotes. There are two bright galaxies at the center of the cluster. The brighter of the two corresponds to the X-ray peak (which unfortunately falls in a chip gap). The second galaxy is to the east, corresponding to the direction of the elongation of the surface brightness. This cluster shows a possible radio relic at $74 \mathrm{MHz}$ in the VLA LowFrequency Sky Survey (VLSS) data. The extended radio emission is northwest of the X-ray peak and extends southwest to northeast $\sim 165 h_{71}^{-1} \mathrm{kpc}$ at the resolution of the VLSS (Cohen et al. 2007).

\section{C.43. RXJ1504.1-0248/RBS 1460}

RX J1504 is the cluster with the highest redshift and X-ray luminosity in HIFLUGCS, and shows the largest classical mass deposition rate. Böhringer et al. (2005) reported the results to the Chandra observation of this cluster. This cluster was originally not included in HIFLUGCS because its X-ray flux is only slightly $(<20 \%)$ above the flux limit. RX J1504 appears only marginally extended in the ROSAT All-Sky Survey. Additionally the galaxy at the center of the X-ray emission is classified as AGN (Machalski \& Condon 1999) and its optical spectrum shows emission lines. It was assumed that even if there is only a small AGN contribution from the central AGN to the total X-ray flux $(\sim 20 \%)$, the cluster would fall below the flux limit. However, the Chandra image reveals that there is actually no significant point source emission at the center of this cluster (Böhringer et al. 2005), which argues against any significant contamination by AGN emission. Therefore, this cluster is included into HIFLUGCS. The BCG features a compact and flat-spectrum radio source (Mittal et al. 2009). This SCC cluster is one of sixteen clusters in which the BCG's central velocity dispersion is not available.

\section{C.44. A2029}

The spectra of the inner annuli fit best to non-solar metalicity ratios, but freeing ratios does not change the best-fit temperatures, so solar ratios were used for simplicity. Clarke et al. (2004) studied the core of this cluster in detail with Chandra.

\section{C.45. A2052}

The central region of this cluster requires a double thermal model out to $\sim 45^{\prime \prime}\left(\sim 32 h_{71}^{-1} \mathrm{kpc}\right)$. Blanton et al. (2001) found prominent $\mathrm{X}$-ray cavities in the original Chandra observation. They determined these cavities to be cospatial with radio lobes from the central radio source.

\section{C.46. MKW3S/WBL 564}

This SCC cluster shows some disruption in the core and bubbles to the south (Mazzotta et al. 2004). MKW3S is one of sixteen clusters in which data about the BCG's central velocity dispersion are not available. This cluster is a member of the Hercules Supercluster.

\section{C.47. A2065}

A2065 is a member of the Corona Borealis Supercluster, in projection close to the Hercules Supercluster but twice as distant. This cluster is one of four clusters on the border between SCC and WCC clusters. Its CCT is $\left(\sim 1.3 h_{71}^{-1 / 2} \mathrm{Gyr}\right)$ is slightly longer than the $1 \mathrm{Gyr}$ cutoff. This cluster shows an inwardly decreasing temperature profile as seen in the SCC clusters. This cluster is one of sixteen clusters in which the BCG's central velocity dispersion is not available. It is one of five clusters where the BCG peculiar velocity is more than $50 \%$ of cluster velocity dispersion. This offset suggests possible sloshing which may have disrupted the CC. Based on the Chandra data, Chatzikos et al. (2006) suggest that the cluster is involved in an unequal mass merger and that one cool core has survived the merger. Feretti \& Giovannini (1994) identified a WAT $\sim 19^{\prime}\left(1.6 h_{71}^{-1} \mathrm{Mpc}\right)$ south south-west of the cluster center. The jets of the WAT are bent away from the center of the cluster. In the NRAO VLA Sky Survey at $1.4 \mathrm{GHz}$ (NVSS Condon et al. 1998), there appears to be a diffuse radio source $\sim 91^{\prime \prime}\left(\sim 124 h_{71}^{-1} \mathrm{kpc}\right)$ to the southwest of the cluster center. It is unclear whether this source is associated with the central radio source.

\section{C.48. A2063}

This WCC cluster appears to have a very regular morphology in X-rays, with some hint of an elongation to the northeast. The BCG resides at the X-ray peak. The NVSS shows three bright radio sources in a line along an axis from southwest to northeast but only the center source is associated with the BCG, while the other two are cospatial with two neighbouring galaxies. As with many WCC clusters this cluster shows a flat central temperature profile and a raised central entropy $K_{0}>50 h_{71}^{-1 / 3} \mathrm{keV} \mathrm{cm}^{2}$. This cluster is close to MKW3S. 


\section{C.49. A2142}

This cluster has a double cold front (Markevitch et al. 2000). The separation between the BCG and the X-ray peak is $\sim 23 h_{71}^{-1} \mathrm{kpc}$ for this cluster, making it one of fourteen with this value $>12 h_{71}^{-1} \mathrm{kpc}$. This is one of sixteen clusters in which no data is available for the BCG's central velocity dispersion. It is possible this cluster hosts a radio halo, but the evidence remains dubious (Giovannini \& Feretti 2000).

\section{C.50. A2147}

A2147 is a member of the Hercules Supercluster. Due to the short observing time combined with the lack of a bright core, we used a $10^{\prime \prime}$ kernel when smoothing to determine the X-ray peak of this NCC cluster. There are three bright galaxies in a line near the core, of which the northernmost (the BCG) is located at the X-ray peak. This is one of the six NCC clusters in which $\dot{M}_{\text {spec }}>0$. The $\mathrm{X}$-ray morphology indicates that it is a merging cluster. The X-ray emission extends toward the south from the peak following the line of the three bright galaxies as well as extending to the southeast. There is, additionally, a sharp drop in the X-ray emission to the northwest. We argue that the observed $\dot{M}_{\text {spec }}$ is not due to cooling, but results from multiple temperatures along the line of sight caused by the merger. Although the cluster has been labeled as a CC cluster in the past (e.g. Henriksen \& White 1996), Sanderson et al. (2006a) found it to be an NCC cluster and likely merger system.

\section{C.51. $A 2163$}

This well-known merging cluster contains the largest known radio halo (Feretti et al. 2001). The separation between the BCG and $\mathrm{X}$-ray peak is $\sim 158 h_{71}^{-1} \mathrm{kpc}$ for this cluster, making it one of eight clusters where this value is $>50 h_{71}^{-1}$. Our measurement of $k T_{\text {vir }}$ $(\sim 16 \mathrm{keV})$ is higher than the value of $\sim 12 \mathrm{keV}$ found by Markevitch \& Vikhlinin (2001) with data from the original, shorter Chandra observation. However, a recent measurement by Vikhlinin et al. (2009), using the same Chandra as we, finds $k T_{\text {vir }} \sim 15 \mathrm{keV}$, more consistent with our result. The difference between our result and Vikhlinin et al. (2009) is barely inconsistent within $1 \sigma$ and is probably due to differences in the techniques used to determine $k T_{\text {vir }}$ in this extremely hot cluster. This is the second most distant and hottest cluster in the HIFLUGCS sample. This is one of sixteen clusters for which data on the BCG's central velocity dispersion are not available, however, since the BCG is not cospatial with the X-ray peak so this information is not important for our analysis.

\section{C.52. A2199}

The central region of this cluster requires a double thermal model out to $\sim 29^{\prime \prime}\left(\sim 17 h_{71}^{-1} \mathrm{kpc}\right)$.

\section{C.53. A2204}

Recently Reiprich et al. (2009) determined the temperature of this cluster out to $\sim r_{200}$ using Suzaku. They find that the temperature declines all the way from $0.3 r_{200}$ to $r_{200}$, consistent with predictions of simulations. This is one of sixteen clusters where data on the BCG's central velocity dispersion is not available.

\section{C.54. A2244}

As with A1651, Donahue et al. (2005) claim it to be a radio quiet CC cluster, but Mittal et al. (2009) detect central radio emission with a bolometric luminosity of $\sim 7 \times 10^{39} h_{71}^{-2} \mathrm{erg} \mathrm{s}^{-1}$. Although this is not particularly luminous, it is consistent with radio activity in other CC clusters (Mittal et al. 2009). Due to elevated entropy in the core, Donahue et al. (2005) concluded a major AGN outburst had disrupted the cooling flow. Like many WCC clusters, this cluster shows a flat temperature profile. However, we point out that the same was true of A1650 until a deeper observation revealed a slight temperature drop in the core. This is one of sixteen clusters in which the central velocity dispersion of the BCG is unavailable.

\section{C.55. A2256}

This well-known merging cluster is the only one of two NCC clusters that shows a systematic temperature decrease in the center. The temperature decrease is the largest of any NCC or WCC cluster. Surprisingly, the separation between the BCG and X-ray peak is $\sim 132 h_{71}^{-1} \mathrm{kpc}$ for this cluster, making it one of eight clusters where this value is $>50 h_{71}^{-1} \mathrm{kpc}$. Since this separation is quite large, the cool gas is not associated with the BCG. It is most likely this gas is the remnant of a CC (perhaps from a merging group) that has been stripped from its central galaxy. This cluster hosts both a radio halo and relic (e.g. Clarke \& Ensslin 2006; Bridle \& Fomalont 1976)

\section{C.56. A2255}

Due to a short exposure time and lack of a bright core, we used a $10^{\prime \prime}$ kernel when smoothing the image to determine the X-ray peak. The separation between the BCG and X-ray peak is $\sim 72 h_{71}^{-1} \mathrm{kpc}$, making it one of eight clusters where this value is $>50 h_{71}^{-1} \mathrm{kpc}$. This cluster BCG also has by far the largest peculiar velocity of any cluster; almost twice the velocity dispersion of the cluster. This cluster hosts both a radio halo and a relic (e.g. Feretti et al. 1997). 


\section{C.57. $A 3667$}

This well-known merging cluster shows a very sharp cold front (Vikhlinin et al. 2001b,a) and two radio relics (e.g. Roettiger et al. 1999). The separation of the BCG and X-ray peak is $\sim 155 h_{71}^{-1} \mathrm{kpc}$ for this clusters making it one of eight clusters where this value is $>50 h_{71}^{-1} \mathrm{kpc}$. This is the only WCC cluster with such a larger separation; however, A3667 is on the border between NCC and WCC clusters, with CCT $\sim 6 h_{71}^{-1}$ Gyr.

\section{C.58. S1101/Sérsic 159-03}

Kaastra et al. (2001) provided a detailed analysis of the XMM-Newton RGS and EPIC data. Recently Werner et al. (2007) have claimed discovery of a diffuse soft excess seen by XMM-Newton and Suzaku and suggest it is of non-thermal origin. This is one of sixteen clusters in which the central velocity dispersion of the BCG is unavailable.

\section{C.59. A2589}

This WCC shows a systematic temperature drop towards the center, albeit rather flat $\left(k T_{0} / k T=0.93\right.$ and $\left.\Gamma=-0.079\right)$. Like A1650, it is on the cusp between SCC and WCC clusters. Zappacosta et al. (2006) studied this cluster with a radio-quiet BCG in detail with XMM-Newton. They find the cluster to be exceptionally relaxed with a gravitating matter profile that fits a NFW profile with $c_{\mathrm{vir}}=6.1 \pm 0.3$ and $M_{\mathrm{vir}}=3.3 \pm 0.3 \times 10^{14} M_{\odot}\left(r_{\mathrm{vir}}=1.74 \pm 0.05 \mathrm{Mpc}\right)$. They conclude that processes during halo formation act against adiabatic contraction. Additionally Buote \& Lewis (2004) studied the original short Chandra observation that suffered from flaring.

Following a method to determine the residual CXB (similar to what is described in Sect 2.3), we measured the surface brightness profile out to $750 h_{71}^{-1} \mathrm{kpc}\left(\sim 0.5 r_{\mathrm{vir}}\right)$. We fit this surface brightness profile to a double- $\beta$ model and the temperature profile to a broken powerlaw. The slope of the inner $k T$ profile was fixed at zero and the outer $k T$ profile fit well to a powerlaw of slope -0.36 with a break radius of $4.2\left(204 h_{71}^{-1} \mathrm{kpc}\right)$. Using the fit to the temperature profile and double $\beta$-model, we find a virial ${ }^{24}$ mass and radius of $M_{\text {vir }}=2.7 \pm 0.8 \times 10^{14} h_{71}^{-1} M_{\odot}$ and $r_{\text {vir }}=1.6 \pm 0.2 h_{71}^{-1} \mathrm{Mpc}$ respectively, consistent with the results of Zappacosta et al. (2006).

\section{C.60. A2597}

McNamara et al. (2001) analyzed the original, short, flared observation of A2597, noting the ghost bubbles. Morris \& Fabian (2005) found high spectral mass deposition rates from the XMM-Newton EPIC and RGS consistent with $\sim 100 M_{\odot} \mathrm{yr}^{-1}$ down to almost $0 \mathrm{keV}$, although the improvement to the spectral fits of the $R G S$ data from the addition of a cooling flow model is marginal. The long Chandra ACIS observation shows a mass deposition rate of $\sim 150 M_{\odot} \mathrm{yr}^{-1}$ down to $\sim 1.3 \mathrm{keV}$ and dropping to $\sim 10 M_{\odot} \mathrm{yr}^{-1} \mathrm{down}$ to $\sim 0 \mathrm{keV}$.

\section{C.61. A2634}

This WCC cluster contains the WAT source 3C465. There is a pair of galaxies (NGC 7720) located at the X-ray peak. An extended bright X-ray halo (radius $=\sim 12^{\prime \prime}$ ), much brighter than the ICM emission, is cospatial with the galaxy pair. The halo seems to be associated with the larger southern galaxy. A2634 is the only CC cluster in the sample with $\dot{M}_{\text {spec }}>\dot{M}_{\text {classical }}$. The temperature profile shows a sudden drop at $\sim 2$ :7 $\left(\sim 100 h_{71}^{-1} \mathrm{kpc}\right)$. Other than NGC 7720, there is no obvious core in A2634 and the elongation of the ICM to the southwest is consistent with a merging cluster. Moreover the inverted temperature profile is more common in NCC clusters than in WCC clusters. We interpret the short cooling time and low $\dot{M}_{\text {classical }}$ as a cool core that has either been disrupted or is in the process of being destroyed by a merger. $\dot{M}_{\text {spec }}$ may reflect the original mass deposition rate, but probably is strongly affected by multitemperature components along the line of sight in a merging system.

\section{C.62. A2657}

This WCC cluster has a slight increase in temperature in the central region. The Chandra image shows a cluster similar to e.g. A1650 and A2244. The central emission peak is clearly visibly but is not as sharply peaked as in SCC clusters. The overall ICM appears to be quite round, with some sloshing features (differently centered X-ray isophotes at different radii) in the central region.

\section{C.63. $A 4038$}

The distance between the BCG and X-ray peak is $\sim 12.4 h_{71}^{-1} \mathrm{kpc}$ for this cluster making it one of fourteen clusters where this separation is $>12 h_{71}^{-1} \mathrm{kpc}$. This cluster hosts a radio relic, close to but not connected to the central radio galaxy (Slee \& Roy 1998 ).

\section{C.64. $A 4059$}

The central region of this cluster requires a double thermal model out to $\sim 22^{\prime \prime}\left(\sim 20 h_{71}^{-1} \mathrm{kpc}\right)$.

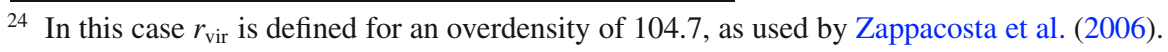


D. S. Hudson et al.: What is a cool-core cluster?

Table 2. Observational parameters.

\begin{tabular}{|c|c|c|c|c|c|c|c|}
\hline$\overline{\text { Cluster }}$ & $\begin{array}{c}\text { RA } \\
(\mathrm{J} 2000) \\
(2)\end{array}$ & $\begin{array}{c}\text { Dec } \\
(\mathrm{J} 2000) \\
(3)\end{array}$ & $\begin{array}{l}\# \text { of } \\
\mathrm{Ob} \\
(4)\end{array}$ & $\begin{array}{l}G T \\
\text { ks } \\
(5)\end{array}$ & $\begin{array}{c}k T_{\text {vir }} \\
\mathrm{keV} \\
(6)\end{array}$ & $\begin{array}{c}Z \\
\text { Solar } \\
(7)\end{array}$ & $\begin{array}{c}\text { Core } \\
\% r_{\text {vir }} \\
(8)\end{array}$ \\
\hline A0085 & $00^{\mathrm{h}} 41^{\mathrm{m}} 50^{\mathrm{s}} 39$ & $-09^{\circ} 18^{\prime} 11^{\prime \prime} .0$ & 9 & 114.7 & $6.00_{-0.11}^{+0.11}$ & $0.38_{-0.05}^{+0.03}$ & $6.2_{-0.8}^{+1.3}$ \\
\hline A0119 & $00^{\mathrm{h}} 56^{\mathrm{m}} 16^{\mathrm{s}} .04$ & $-01^{\circ} 15^{\prime} 20 .^{\prime} 6$ & 1 & 11.4 & $5.73_{-0.30}^{+0.31}$ & $0.48_{-0.11}^{+0.11}$ & $0^{-0.8}$ \\
\hline A0133 & $01^{\mathrm{h}} 02^{\mathrm{m}} 41^{\mathrm{s}} .78$ & $-21^{\circ} 52^{\prime} 56^{\prime \prime} 0$ & 3 & 120.5 & $3.96_{-0.10}^{+0.08}$ & $0.41_{-0.07}^{+0.11}$ & $4.5_{-0.5}^{+0.6}$ \\
\hline NGC 0507 & $01^{\mathrm{h}} 23^{\mathrm{m}} 39^{\mathrm{s}} .82$ & $+33^{\circ} 15^{\prime} 21^{\prime \prime} .5$ & 1 & 43.5 & $1.44_{-0.10}^{+0.10}$ & $0.43^{+0.11}$ & $3.4^{-0.5}$ \\
\hline A0262 & $01^{\mathrm{h}} 52^{\mathrm{m}} 46^{\mathrm{s}} .23$ & $+36^{\circ} 09^{\prime} 14^{\prime \prime} .9$ & 1 & 28.0 & $2.44_{-0.04}^{+0.03}$ & $0.60_{-0.03}^{+0.04}$ & $2.0_{-0.1}^{+0.2}$ \\
\hline A 0400 & $02^{\mathrm{h}} 57^{\mathrm{m}} 41^{\mathrm{s}} .59$ & $+06^{\circ} 01^{\prime} 37^{\prime \prime} .4$ & 1 & 21.5 & $2.26_{-0.012}^{+0.04}$ & $\begin{array}{l}0.50_{-0.06}^{+0.03} \\
0.07\end{array}$ & $0^{-0.1}$ \\
\hline A0399 & $02^{\mathrm{h}} 57^{\mathrm{m}} 53^{\mathrm{s}} .45$ & $+13^{\circ} 01^{\prime} 52^{\prime \prime} .8$ & 1 & 49.0 & $6.70_{-0.14}^{+0.12}$ & $0.30_{-0.04}^{+0.05}$ & 0 \\
\hline A0401 & $02^{\mathrm{h}} 58^{\mathrm{m}} 56^{\mathrm{s}} .66$ & $+13^{\circ} 34^{\prime} 39^{\prime} .8$ & 2 & 29.6 & $8.51_{-0.22}^{+0.34}$ & $0.34_{-0.06}^{+0.06}$ & 0 \\
\hline A3112 & $03^{\mathrm{h}} 17^{\mathrm{m}} 57^{\mathrm{s}} .65$ & $-44^{\circ} 14^{\prime} 18^{\prime \prime} .3$ & 5 & 90.5 & $4.73_{-0.12}^{+0.11}$ & $0.38_{-0.05}^{+0.06}$ & $5.2_{-0.5}^{+0.8}$ \\
\hline NGC 1399 & $03^{\mathrm{h}} 38^{\mathrm{m}} 29.10$ & $-35^{\circ} 27^{\prime} 00^{\prime} 9$ & 12 & 429.7 & $1.34_{-0.00}^{-0.12}$ & $0.27_{-0.00}^{-0.05}$ & $2.0_{-0.1}^{+0.5}$ \\
\hline $2 \mathrm{~A} 0335^{\dagger}$ & $03^{\mathrm{h}} 38^{\mathrm{m}} 41^{\mathrm{s}} .14$ & $+09^{\circ} 58^{\prime} 01^{\prime \prime} 99$ & 1 & 19.7 & $3.53_{-0.13}^{+0.00}$ & $0.54_{-0.05}^{+0.00}$ & $5.5_{-0.4}^{+0.1}$ \\
\hline IIIZw54 & $03^{\mathrm{h}} 41^{\mathrm{m}} 17^{\mathrm{s}} .64$ & $+15^{\circ} 23^{\prime} 37^{\prime \prime} .1$ & 1 & 23.3 & $2.50_{-0.06}^{+0.13}$ & $0.37_{-0.04}^{-0.05}$ & $0^{-0.6}$ \\
\hline A3158 & $03^{\mathrm{h}} 42^{\mathrm{m}} 52^{\mathrm{s}} .27$ & $-53^{\circ} 37^{\prime} 55^{\prime \prime} .5$ & 2 & 55.7 & $4.99_{-0.07}^{+0.00}$ & $0.41_{-0.03}^{+0.04}$ & 0 \\
\hline A0478 & $04^{\mathrm{h}} 13^{\mathrm{m}} 25^{\mathrm{s}} .15$ & $+10^{\circ} 27^{\prime} 53^{\prime \prime} .8$ & 8 & 101.4 & $7.34_{-0.19}^{+0.18}$ & $0.31_{-0.03}^{+0.03}$ & $5.2_{-06}^{+0.7}$ \\
\hline NGC 1550 & $04^{\mathrm{h}} 19^{\mathrm{m}} 37^{\mathrm{s}} .97$ & $+02^{\circ} 24^{\prime} 36^{\prime \prime} .2$ & 4 & 107.6 & $1.34_{-0.00}^{+0.19}$ & $0.25_{-0.01}^{+0.03}$ & $2.0_{-0.1}^{+0.6}$ \\
\hline EXO0422 ${ }^{\dagger}$ & $04^{\mathrm{h}} 25^{\mathrm{m}} 51^{\mathrm{s}} .24$ & $-08^{\circ} 33^{\prime} 37^{\prime \prime} .9$ & 1 & 9.8 & $2.93_{-0.12}^{+0.00}$ & $0.36_{-0.07}^{+0.01}$ & $3.2_{-1.4}^{+2.1}$ \\
\hline A3266 & $04^{\mathrm{h}} 31^{\mathrm{m}} 13^{\mathrm{s}} .13$ & $-61^{\circ} 27^{\prime} 11^{\prime \prime} .0$ & 1 & 29.5 & $9.45_{-0.36}^{+0.35}$ & $0.26_{-0.06}^{+0.06}$ & 0 \\
\hline A0496 & $04^{\mathrm{h}} 33^{\mathrm{m}} 37^{\mathrm{s}} .95$ & $-13^{\circ} 15^{\prime} 39^{\prime} \cdot 9$ & 2 & 66.4 & $4.86^{-0.050}$ & $0.66_{-0.03}^{+0.06}$ & $4.8_{-0.3}^{+0.3}$ \\
\hline A3376 & $06^{\mathrm{h}} 02^{\mathrm{m}} 08^{\mathrm{s}} .64$ & $-39^{\circ} 56^{\prime} 48^{\prime \prime} .5$ & 2 & 64.2 & $\begin{array}{l}3.80_{-0.10}^{+0.06} \\
+0.11\end{array}$ & $0.31_{-0.04}^{-0.03}$ & 0 \\
\hline A3391 & $06^{\mathrm{h}} 26^{\mathrm{m}} 20^{\mathrm{s}} .50$ & $-53^{\circ} 41^{\prime} 37^{\prime \prime} .0$ & 1 & 17.1 & $5.77_{-0.36}^{+0.30}$ & $0.33_{-0.09}^{+0.13}$ & 0 \\
\hline A3395s & $06^{\mathrm{h}} 26^{\mathrm{m}} 49^{\mathrm{s}} .74$ & $-54^{\circ} 32^{\prime} 33^{\prime \prime} .^{\prime} 6$ & 1 & 21.0 & $4.82_{-0.26}^{-0.36}$ & $0.09_{-0.08}^{-0.09}$ & 0 \\
\hline A0576 & $07^{\mathrm{h}} 21^{\mathrm{m}} 30^{\mathrm{s}} .26$ & $+55^{\circ} 45^{\prime} 50^{\prime} .6$ & 1 & 27.0 & $4.09_{-0.10}^{+0.08}$ & $0.59_{-0.06}^{+0.08}$ & 0 \\
\hline A0754 & $09^{\mathrm{h}} 09^{\mathrm{m}} 16^{\mathrm{s}} .66$ & $-09^{\circ} 41^{\prime} 20^{\prime} .8$ & 1 & 44.1 & $11.13_{-0.33}^{+0.39}$ & $0.30_{-0.06}^{+0.05}$ & 0 \\
\hline A0780 & $09^{\mathrm{h}} 18^{\mathrm{m}} 6^{\mathrm{s}} .09$ & $-12^{\circ} 05^{\prime} 45^{\prime \prime} .0$ & 2 & 186.7 & $3.45_{-0.09}^{+0.08}$ & $0.27_{-0.03}^{+0.06}$ & $13.0_{-1.3}^{+2.1}$ \\
\hline A1060 & $10^{\mathrm{h}} 36^{\mathrm{m}} 42^{\mathrm{s}} .75$ & $-27^{\circ} 31^{\prime} 42^{\prime \prime} 0$ & 1 & 31.4 & $3.16_{-0.04}^{+0.09}$ & $0.47_{-0.03}^{-0.03}$ & $0^{-1.3}$ \\
\hline A1367 & $11^{\mathrm{h}} 45^{\mathrm{m}} 00^{\varsigma} .29$ & $+19^{\circ} 40^{\prime} 30^{\prime} \cdot 2$ & 2 & 68.7 & $3.58_{-0.06}^{+0.06}$ & $0.35_{-0.02}^{+0.04}$ & 0 \\
\hline MKW4 & $12^{\mathrm{h}} 04^{\mathrm{m}} 27^{\mathrm{s}} .08$ & $+01^{\circ} 53^{\prime} 46^{\prime \prime} 1$ & 1 & 30.1 & $2.01_{-0.04}^{+0.00}$ & $0.65_{-0.04}^{+0.02}$ & $1.8_{-02}^{+0.4}$ \\
\hline $\mathrm{ZwCl1215^{ \dagger }}$ & $12^{\mathrm{h}} 17^{\mathrm{m}} 41^{\mathrm{s}} .71$ & $+03^{\circ} 39^{\prime} 18^{\prime \prime} .4$ & 1 & 11.9 & $6.27_{-0.29}^{+0.04}$ & $0.25_{-0.08}^{+0.09}$ & $0^{-0.2}$ \\
\hline NGC 4636 & $12^{\mathrm{h}} 42^{\mathrm{m}} 49^{\mathrm{s}} .91$ & $+02^{\circ} 41^{\prime} 12^{\prime \prime} .6$ & 2 & 150.3 & $0.90_{-0.02}^{+0.02}$ & $0.20_{-0.02}^{+0.02}$ & $2.0_{-0.1}^{+0.1}$ \\
\hline A3526 & $12^{\mathrm{h}} 48^{\mathrm{m}} 48^{\mathrm{s}} .85$ & $-41^{\circ} 18^{\prime} 43^{\prime \prime} .8$ & 7 & 288.6 & $3.92_{-0.02}^{-0.02}$ & $0.60_{-0.01}^{+0.02}$ & $3.16_{-0.05}^{+0.105}$ \\
\hline A1644 & $12^{\mathrm{h}} 57^{\mathrm{m}} 11^{\mathrm{s}} .79$ & $-17^{\circ} 24^{\prime} 32^{\prime \prime} .3$ & 2 & 70.3 & $5.09_{-0.09}^{+0.09}$ & $0.37_{-0.04}^{+0.01}$ & $5.6_{-0.8}^{+0.65}$ \\
\hline A1650 & $12^{\mathrm{h}} 58^{\mathrm{m}} 41^{\mathrm{s}} .48$ & $-01^{\circ} 45^{\prime} 42^{\prime \prime} 7$ & 7 & 225.3 & $5.81_{-0.07}^{+0.06}$ & $0.37_{-0.02}^{+0.03}$ & $2.2_{-0.6}^{+1.8}$ \\
\hline A1651 & $12^{\mathrm{h}} 59^{\mathrm{m}} 22^{\mathrm{s}} .16$ & $-04^{\circ} 11^{\prime} 49^{\prime} 2$ & 1 & 9.3 & $\begin{array}{l}-0.07 \\
6.34_{-0.27}^{+0.07}\end{array}$ & $0.47_{-0.09}^{-0.02}$ & $0^{-0.6}$ \\
\hline A1656 & $12^{\mathrm{h}} 59^{\mathrm{m}} 35^{\mathrm{s}} .73$ & $+27^{\circ} 57^{\prime} 34^{\prime \prime} .9$ & 1 & 29.8 & $9.15_{-0.16}^{+0.17}$ & $0.33_{-0.03}^{+0.03}$ & 0 \\
\hline NGC 5044 & $13^{\mathrm{h}} 15^{\mathrm{m}} 23^{\mathrm{s}} .88$ & $-16^{\circ} 23^{\prime} 06^{\prime \prime} 8$ & 1 & 15.8 & $1.22_{-0.04}^{+0.03}$ & $0.25_{-0.02}^{+0.04}$ & $5.4_{-0.1}^{+1.3}$ \\
\hline A1736 & $13^{\mathrm{h}} 26^{\mathrm{m}} 51^{\mathrm{s}} .87$ & $-27^{\circ} 10^{\prime} 26^{\prime \prime} .8$ & 1 & 14.8 & $3.12_{-0.12}^{-0.04}$ & $0.34_{-0.06}^{+0.02}$ & $0^{-0.1}$ \\
\hline A3558 & $13^{\mathrm{h}} 27^{\mathrm{m}} 56^{\mathrm{s}} .89$ & $-31^{\circ} 29^{\prime} 43^{\prime \prime} .2$ & 1 & 9.9 & $4.95_{-0.15}^{+0.12}$ & $0.27_{-0.06}^{+0.06}$ & $5.8_{-2.3}^{+6.6}$ \\
\hline A 3562 & $13^{\mathrm{h}} 33^{\mathrm{m}} 37^{\mathrm{s}} .29$ & $-31^{\circ} 40^{\prime} 17^{\prime \prime} .0$ & 1 & 19.4 & $4.43_{-0.16}^{-0.15}$ & $0.44_{-0.07}^{-0.06}$ & $0^{-2.3}$ \\
\hline A3571 & $13^{\mathrm{h}} 47^{\mathrm{m}} 28^{\mathrm{s}} .32$ & $-32^{\circ} 51^{\prime} 57^{\prime \prime} .5$ & 1 & 25.9 & $7.00_{-0.12}^{+0.16}$ & $0.45_{-0.03}^{+0.04}$ & 0 \\
\hline A1795 & $13^{\mathrm{h}} 48^{\mathrm{m}} 52^{\mathrm{s}} .58$ & $+26^{\circ} 35^{\prime} 33^{\prime \prime} .1$ & 12 & 173.5 & $6.08_{-0.07}^{+0.07}$ & $0.28_{-0.02}^{+0.02}$ & $6.9_{-0.5}^{+0.6}$ \\
\hline A3581 & $14^{\mathrm{h}} 07^{\mathrm{m}} 30^{\mathrm{s}} .19$ & $-27^{\circ} 01^{\prime} 10^{\prime} .5$ & 1 & 6.2 & $1.97_{-0.07}^{+0.07}$ & $0.53_{-0.07}^{+0.08}$ & $3.0_{-03}^{+0.4}$ \\
\hline MKW8 & $14^{\mathrm{h}} 40^{\mathrm{m}} 43^{\mathrm{s}} .08$ & $+03^{\circ} 27^{\prime} 57^{\prime \prime} .7$ & 1 & 23.1 & $3.00_{-0.12}^{+0.12}$ & $0.45_{-0.07}^{+0.07}$ & 0 \\
\hline $\mathrm{RXJ} 1504^{\dagger}$ & $15^{\mathrm{h}} 04^{\mathrm{m}} 07^{\mathrm{s}} .52$ & $-02^{\circ} 48^{\prime} 16^{\prime \prime} .8$ & 2 & 52.5 & $9.53_{-1.16}^{+1.139}$ & $0.38_{-0.22}^{+0.01}$ & $12.1_{-3.6}^{+17.3}$ \\
\hline A2029 & $15^{\mathrm{h}} 10^{\mathrm{m}} 56^{\mathrm{s}} .06$ & $+05^{\circ} 44^{\prime} 41^{\prime \prime} .4$ & 3 & 107.0 & $8.26_{-0.09}^{+0.09}$ & $0.38_{-0.02}^{+0.02}$ & $3.7_{-0.4}^{+0.5}$ \\
\hline A2052 & $15^{\mathrm{h}} 16^{\mathrm{m}} 43^{\mathrm{s}} .51$ & $+07^{\circ} 01^{\prime} 19^{\prime} .8$ & 2 & 163.9 & $3.35_{-0.02}^{+0.02}$ & $0.66_{-0.02}^{+0.02}$ & $2.55_{-0.07}^{+0.45}$ \\
\hline MKW3S & $15^{\mathrm{h}} 21^{\mathrm{m}} 51^{\mathrm{s}} .75$ & $+07^{\circ} 42^{\prime} 28^{\prime \prime} .7$ & 1 & 56.7 & $3.90_{-0.09}^{+0.02}$ & $0.41_{-0.04}^{-0.02}$ & $6.2_{-1.8}^{+2.07}$ \\
\hline A2065 & $15^{\mathrm{h}} 22^{\mathrm{m}} 29^{\mathrm{s}} .32$ & $+27^{\circ} 42^{\prime} 22^{\prime \prime} 2$ & 1 & 49.5 & $5.40_{-0.11}^{+0.09}$ & $0.29_{-0.04}^{+0.04}$ & $5.1_{-1.7}^{+4.8}$ \\
\hline A2063 & $15^{\mathrm{h}} 23^{\mathrm{m}} 05^{\mathrm{s}} .11$ & $+08^{\circ} 36^{\prime} 26^{\prime \prime} 9$ & 2 & 28.5 & $3.77_{-0.06}^{+0.06}$ & $0.60_{-0.04}^{+0.04}$ & $0^{-1.7}$ \\
\hline A2142 & $15^{\mathrm{h}} 58^{\mathrm{m}} 20^{\mathrm{s}} .65$ & $+27^{\circ} 13^{\prime} 49^{\prime} .2$ & 1 & 44.8 & $8.40_{-0.76}^{+1.00}$ & $0.32_{-0.23}^{+0.24}$ & $>24.0$ \\
\hline A2147 & $16^{\mathrm{h}} 02^{\mathrm{m}} 16^{\mathrm{s}} .78$ & $+15^{\circ} 58^{\prime} 25^{\prime \prime} .6$ & 1 & 17.0 & $4.07_{-0.12}^{+0.11}$ & $0.33_{-0.06}^{+0.06}$ & 0 \\
\hline
\end{tabular}


Table 2. continued.

\begin{tabular}{|c|c|c|c|c|c|c|c|}
\hline Cluster & $\begin{array}{c}\text { RA } \\
(\mathrm{J} 2000) \\
(2)\end{array}$ & $\begin{array}{c}\text { Dec } \\
(\mathrm{J} 2000) \\
(3)\end{array}$ & $\begin{array}{l}\# \text { of } \\
\mathrm{Ob} \\
(4)\end{array}$ & $\begin{array}{l}G T \\
\text { ks } \\
(5)\end{array}$ & $\begin{array}{c}k T_{\text {vir }} \\
\mathrm{keV} \\
(6)\end{array}$ & $\begin{array}{c}Z \\
\text { Solar } \\
(7)\end{array}$ & $\begin{array}{c}\text { Core } \\
\% r_{\text {vir }} \\
(8)\end{array}$ \\
\hline A2163 & $16^{\mathrm{h}} 15^{\mathrm{m}} 46^{\mathrm{s}} .69$ & $-06^{\circ} 09^{\prime} 00^{\prime} 3$ & 2 & 80.3 & $15.91_{-0.81}^{+0.81}$ & $0.32_{-0.06}^{+0.06}$ & 0 \\
\hline A2199 & $16^{\mathrm{h}} 28^{\mathrm{m}} 38^{\mathrm{s}} .32$ & $+39^{\circ} 33^{\prime} 01^{\prime \prime} .2$ & 1 & 16.6 & $\begin{array}{l}-0.81 \\
4.37_{-0.07}^{+0.07}\end{array}$ & $0.52_{-0.03}^{+0.06}$ & $1.8_{-06}^{+0.1}$ \\
\hline A2204 & $16^{\mathrm{h}} 32^{\mathrm{m}} 46^{\mathrm{s}} .94$ & $+05^{\circ} 34^{\prime} 31^{\prime \prime} .3$ & 2 & 18.6 & $8.92_{-0.61}^{+0.01}$ & $0.28_{-0.11}^{+0.11}$ & $5.4_{-1.4}^{+1.8}$ \\
\hline A2244 & $17^{\mathrm{h}} 02^{\mathrm{m}} 42^{\mathrm{s}} .68$ & $+34^{\circ} 03^{\prime} 39^{\prime} \cdot 3$ & 1 & 56.0 & $5.78_{-0.11}^{+0.61}$ & $0.43_{-0.03}^{+0.11}$ & $0^{-1.4}$ \\
\hline A2256 & $17^{\mathrm{h}} 03^{\mathrm{m}} 14^{\mathrm{s}} .26$ & $+78^{\circ} 38^{\prime} 59^{\prime} .9$ & 1 & 8.0 & $7.61_{-0.63}^{+0.65}$ & $0.26_{-0.17}^{+0.18}$ & $14.4^{+8.7}$ \\
\hline A 2255 & $17^{\mathrm{h}} 12^{\mathrm{m}} 34^{\mathrm{s}} .15$ & $+64^{\circ} 04^{\prime} 11^{\prime \prime} .5$ & 1 & 39.6 & $5.81_{-0.20}^{+0.03}$ & $0.27_{-0.07}^{+0.17}$ & $0^{-4.1}$ \\
\hline A 3667 & $20^{\mathrm{h}} 12^{\mathrm{m}} 42^{\mathrm{s}} .66$ & $-56^{\circ} 50^{\prime} 48^{\prime \prime} 6$ & 7 & 485.3 & $6.39_{-0.04}^{+0.04}$ & $0.35_{-0.01}^{+0.01}$ & $1.2_{-0.5}^{+0.7}$ \\
\hline S1101 & $23^{\mathrm{h}} 13^{\mathrm{m}} 58^{\mathrm{s}} .40$ & $-42^{\circ} 43^{\prime} 31^{\prime \prime} .0$ & 1 & 7.9 & $2.57_{-0.13}^{+0.04}$ & $0.23_{-0.06}^{+0.01}$ & $6.4_{-31}^{+4.5}$ \\
\hline A2589 & $23^{\mathrm{h}} 23^{\mathrm{m}} 57^{\mathrm{s}} .40$ & $+16^{\circ} 46^{\prime} 37^{\prime \prime} .9$ & 3 & 76.7 & $3.89_{-0.05}^{-0.13}$ & $0.80_{-0.04}^{+0.06}$ & $1.9_{-0.2}^{+1.1}$ \\
\hline A2597 & $23^{\mathrm{h}} 25^{\mathrm{m}} 19^{\mathrm{s}} .93$ & $-12^{\circ} 07^{\prime} 27^{\prime \prime} .5$ & 2 & 112.0 & $4.05_{-0.07}^{+0.07}$ & $0.38_{-0.04}^{+0.04}$ & $5.8_{-06}^{+1.0}$ \\
\hline A2634 & $23^{\mathrm{h}} 38^{\mathrm{m}} 29^{\mathrm{s}} .25$ & $+27^{\circ} 01^{\prime} 54^{\prime \prime} .2$ & 1 & 48.7 & $3.19_{-0.11}^{-0.07}$ & $0.34_{-0.05}^{+0.04}$ & $0^{-0.6}$ \\
\hline A2657 & $23^{\mathrm{h}} 44^{\mathrm{m}} 57^{\mathrm{s}} .48$ & $+09^{\circ} 11^{\prime} 31^{\prime \prime} .0$ & 1 & 16.1 & $3.52_{-0.11}^{+0.11}$ & $0.38_{-0.06}^{+0.06}$ & 0 \\
\hline A4038 & $23^{\mathrm{h}} 47^{\mathrm{m}} 43^{\mathrm{s}} .18$ & $-28^{\circ} 08^{\prime} 31^{\prime \prime} .2$ & 2 & 40.0 & $3.14_{-0.04}^{+0.11}$ & $\begin{array}{l}.0 .0 .06 \\
0.50_{-0.03}^{+0.03}\end{array}$ & 0 \\
\hline A4059 & $23^{\mathrm{h}} 57^{\mathrm{m}} 00^{\mathrm{s}} .93$ & $-34^{\circ} 45^{\prime} 33^{\prime \prime} .3$ & 2 & 109.9 & $4.22_{-0.03}^{+0.04}$ & $0.66_{-0.02}^{+0.03}$ & $2.4_{-0.3}^{+0.1}$ \\
\hline \multicolumn{4}{|c|}{${ }^{\dagger}$ Abbreviated Cluster Names: } & \multicolumn{4}{|c|}{ Alternative Cluster Names: } \\
\hline \multicolumn{4}{|c|}{ 2A0335: 2A 0335+096 } & \multicolumn{4}{|c|}{ Coma: A1656 } \\
\hline \multicolumn{4}{|c|}{ EXO0422: EXO 0422-086 } & \multicolumn{4}{|c|}{ Fornax: NGC 1399} \\
\hline \multicolumn{4}{|c|}{ ZwCl1215: ZwCl 1215.1+0400 } & \multicolumn{4}{|c|}{ Centaurus: A3526 } \\
\hline \multicolumn{4}{|c|}{ RX J1504: RX J1504.1-0248 } & & & & \\
\hline
\end{tabular}

Notes. (1) cluster name; (2) Right Ascension and (3) declination of the X-ray emission peak; (4) number of observations used; (5) the total good time; (6) the fitted cluster virial temperature; (7) the fitted cluster metalicity and (8) The size of the region with $T<T_{\text {vir }}$ (see Sect. 2.3). 


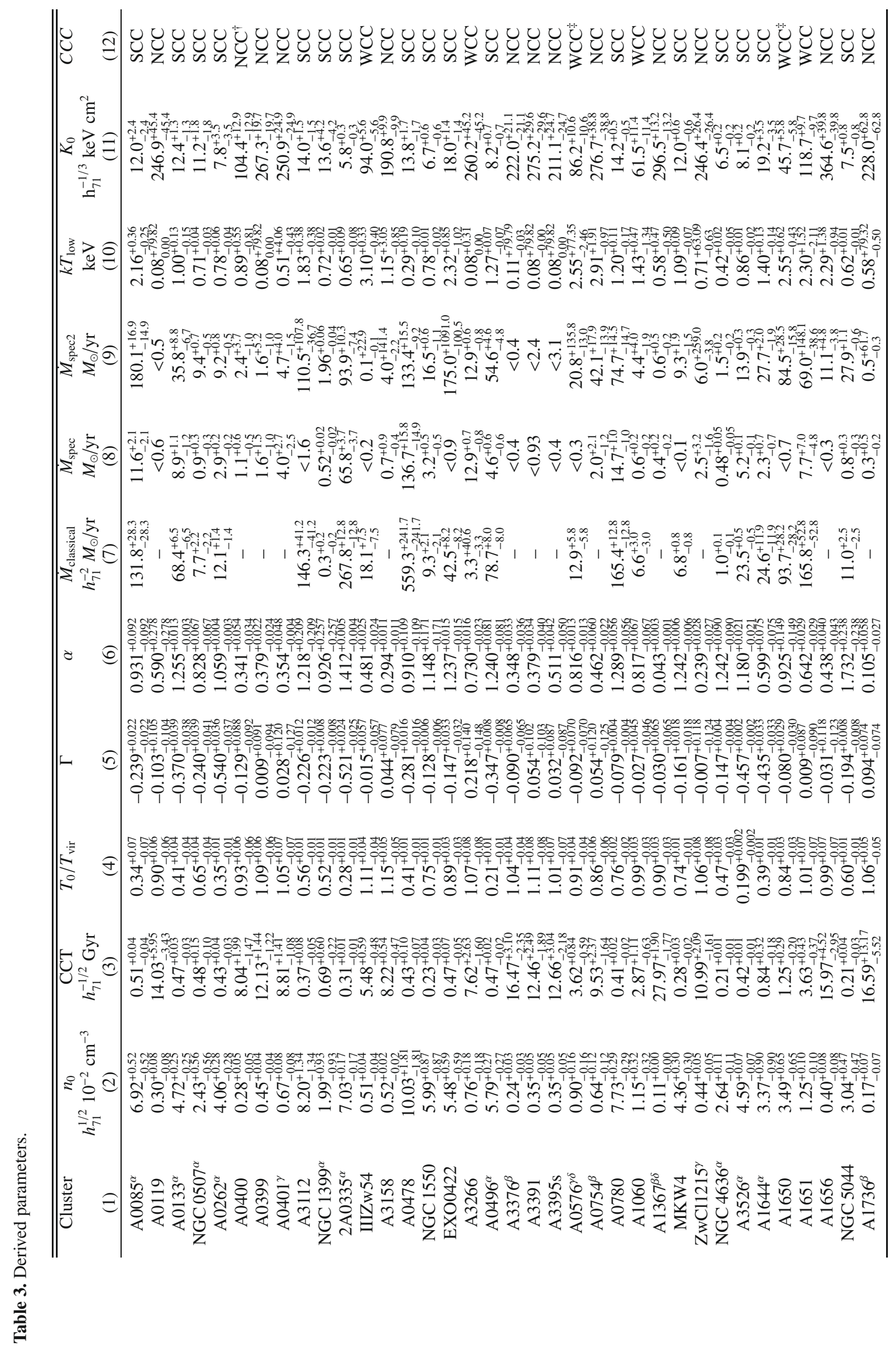



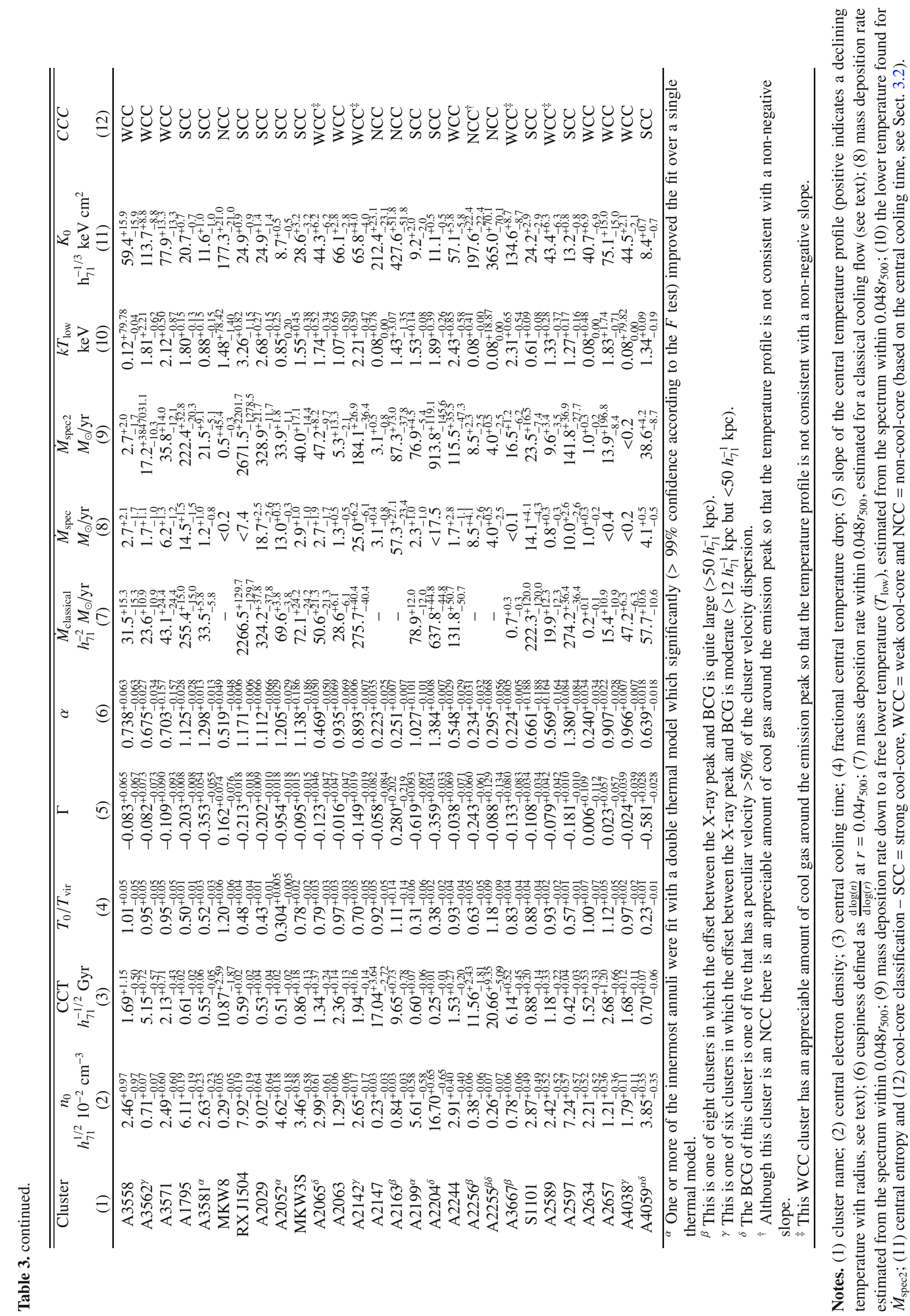

Page 40 of 40 